\title{
PROJECTIVE GEOMETRIES ARISING FROM ELEKES-SZABÓ PROBLEMS
}

\author{
MARTIN BAYS AND EMMANUEL BREUILLARD
}

\begin{abstract}
We generalise the Elekes-Szabó theorem to arbitrary arity and dimension and characterise the complex algebraic varieties without power saving. The characterisation involves certain algebraic subgroups of commutative algebraic groups endowed with an extra structure arising from a skew field of endomorphisms. We also extend the Erdős-Szemerédi sum-product phenomenon to elliptic curves. Our approach is based on Hrushovski's framework of pseudo-finite dimensions and the abelian group configuration theorem.
\end{abstract}

\section{INTRODUCTION}

Let $V \subset \mathbb{C}^{n}$ be an irreducible algebraic set over $\mathbb{C}$, let $N \in \mathbb{N}$, and let $X_{i} \subset \mathbb{C}$ with $\left|X_{i}\right| \leq N, i=1 \ldots n$. Then it is easy to see that

$$
\left|V \cap \prod_{i=1}^{n} X_{i}\right| \leq O_{V}\left(N^{\operatorname{dim} V}\right) .
$$

Indeed, this follows inductively from the observation that there exists an algebraic subset $W \subset V$ of lesser dimension and a co-ordinate projection of the complement $V \backslash W \rightarrow \mathbb{C}^{\operatorname{dim} V}$ with fibres of finite size bounded by a constant.

Say $V$ admits no power-saving if the exponent $\operatorname{dim} V$ is optimal, i.e. if for no $\epsilon>0$ do we have a bound $\left|V \cap \prod_{i=1}^{n} X_{i}\right| \leq O_{V, \epsilon}\left(N^{\operatorname{dim} V-\epsilon}\right)$ as the $X_{i}$ vary among finite subsets of $\mathbb{C}$ of size $\leq N$.

In an influential paper Elekes and Szabó [ES12] classified the varieties which admit no power-saving in the case $n=3$. In order to state their main theorem, we first need the following definition:

Definition 1.1. A generically finite algebraic correspondence between irreducible algebraic varieties $V$ and $V^{\prime}$ is a closed irreducible subvariety of the product $\Gamma \subset V \times V^{\prime}$ such that the projections $\pi_{V}(\Gamma) \subset V$ and $\pi_{V^{\prime}}(\Gamma) \subset V^{\prime}$ are Zariski dense, and $\operatorname{dim}(\Gamma)=\operatorname{dim}(V)=\operatorname{dim}\left(V^{\prime}\right)$.

Suppose $W_{1}, \ldots, W_{n}$ and $W_{1}^{\prime}, \ldots, W_{n}^{\prime}$ are irreducible algebraic varieties, and $V \subset$ $\prod_{i=1}^{n} W_{i}$ and $V^{\prime} \subset \prod_{i=1}^{n} W_{i}^{\prime}$ are irreducible subvarieties. Then we say $V$ and $V^{\prime}$ are in co-ordinatewise correspondence if there is a generically finite algebraic correspondence $\Gamma \subset V \times V^{\prime}$ and a permutation $\sigma \in \operatorname{Sym}(n)$ such that for each $i$, the closure of the projection $\left(\pi_{i} \times \pi_{\sigma i}^{\prime}\right)(\Gamma) \subset W_{i} \times W_{\sigma i}^{\prime}$ is a generically finite algebraic correspondence (between the closure of $\pi_{i}(V)$ and the closure of $\pi_{\sigma i}^{\prime}\left(V^{\prime}\right)$ ).

Theorem 1.2 (Elekes-Szabó [ES12]). An irreducible surface $V \subset \mathbb{C}^{3}$ admits no power-saving if and only if either

(i) $V \subset \mathbb{C}^{3}$ is in co-ordinatewise correspondence with the graph $\Gamma=\{(g, h, g+h)$ : $g, h \in G\} \subset G^{3}$ of the group operation of a 1-dimensional connected complex algebraic group $G$,

(ii) or $V$ projects to a curve, i.e. $\operatorname{dim}\left(\pi_{i j}(V)\right)=1$ for some $i \neq j \in\{1,2,3\}$.

Date: June 3, 2021. 
Here we generalise these results to arbitrary $n$ and $V \subset \mathbb{C}^{n}$.

Definition 1.3. An irreducible algebraic set $V \subset \mathbb{C}^{n}$ is special if it is in coordinatewise correspondence with a product $\prod_{i} H_{i} \leq \prod_{i} G_{i}^{n_{i}}$ of connected subgroups $H_{i}$ of powers $G_{i}^{n_{i}}$ of 1 -dimensional complex algebraic groups, where $\sum_{i} n_{i}=$ $n$.

We prove:

Theorem 1.4. An irreducible algebraic set $V \subset \mathbb{C}^{n}$ admits no power-saving if and only if it is special.

The case of Theorem 1.4 with $V \subset \mathbb{C}^{3}$ and $\operatorname{dim}(V)=2$ is precisely Theorem 1.2. Indeed it is easy to verify that $V$ is special if and only if it is either of the form $(i)$ or of the form $(i i)$. The latter occurs exactly when the special subgroup $H \leq G^{3}$ can be taken to be a diagonal subgroup $\left\{x_{i}=x_{j}\right\}$, while the curve $\pi_{i j}(V)$ gives the correspondence.

The case $V \subset \mathbb{C}^{4}$ with $\operatorname{dim}(V)=3$ is a consequence of the results of [RSdZ18].

A slightly stonger version of the case $V \subset \mathbb{C}^{n}$ with $\operatorname{dim}(V)=n-1$, asking also for some uniformity in the power-saving (c.f. Remark 1.14), was conjectured by de Zeeuw in [dZ18, Conjecture 4.3].

The case $V \subset \mathbb{C}^{4}$ with $\operatorname{dim}(V)=2$ solves [dZ18, Problem 4.4].

Example 1.5. $V:=\left\{(x, y, z, w) \in \mathbb{C}^{4}: x z w=1=y z^{2} w^{2}\right\}$ is special because it is a subgroup of $\left(\mathbb{C}^{*}\right)^{4}$, and geometric progressions witness that it admits no powersaving: setting $X=\left\{2^{k}:-M \leq k \leq M\right\}$, we find $\left|V \cap X^{4}\right| \geq \Omega\left(M^{2}\right) \geq \Omega\left(|X|^{2}\right)$.

Example 1.6. Let $E \subset \mathbb{P}^{2}(\mathbb{C})$ be an elliptic curve, say defined by $\left\{y^{2}=x(x-1)(x-\right.$ $\lambda)\}$. Then taking $x$ co-ordinates yields a surface $V \subset \mathbb{C}^{3}$ in co-ordinatewise correspondence with the graph $\Gamma_{+} \subset E^{3}$ of the elliptic curve group law, and arithmetic progressions in $E$ witness that $V$ admits no power-saving. This demonstrates the necessity of taking correspondences in the definition of special.

To demonstrate the necessity of taking products, suppose $E^{\prime} \subset \mathbb{P}^{2}(\mathbb{C})$ is another elliptic curve. Then taking $x$ co-ordinates yields a 4-dimensional subvariety $W \subset \mathbb{C}^{6}$ in co-ordinatewise correspondence with the product $\Gamma_{+} \times \Gamma_{+^{\prime}} \subset E^{3} \times E^{\prime 3}$ of the graphs of the two group laws, and again arithmetic progressions witness that $W$ admits no power-saving. But if $E^{\prime}$ is not isogenous to $E$, then $W$ is not in coordinatewise correspondence with a subgroup of a power of a single elliptic curve (see Fact 2.13).

In fact we obtain a more general result, with arbitrary varieties in place of the complex co-ordinates. Again, this generalises the corresponding result of [ES12], who considered the case of a subvariety $V$ of $\mathbb{C}^{d} \times \mathbb{C}^{d} \times \mathbb{C}^{d}$ of dimension $2 d$ and with dominant projections to pairs of co-ordinates, and showed that $V$ must be in correspondence with the graph of multiplication of some algebraic group $G$. In [BW16] it was noted that this group must be commutative. Theorem 1.11 below gives a complete classification of the subvarieties without power saving, showing in particular that the groups involved must be commutative. To state the result, we first introduce the following definition.

Definition 1.7. Let $W$ be a complex variety. Let $C, \tau \in \mathbb{N}$ with $C \geq \tau$. A finite subset $X \subset W$ is in coarse $(C, \tau)$-general position in $W$ if for any proper irreducible complex closed subvariety $W^{\prime} \subsetneq W$ of complexity at most $C$, we have $\left|W^{\prime} \cap X\right| \leq|X|^{\frac{1}{\tau}}$. When $C=\tau$ we will simply say that $X$ is $\tau$ - $\boldsymbol{c g} \boldsymbol{p}$ in $W$.

The notion of the complexity of a subvariety of a fixed variety is defined in full generality in 2.1 .10 below. In the case that $W$ is affine, $W^{\prime} \subset W$ has complexity at most $C$ if it can be defined as the zero set of polynomials of degree at most $C$. 
Let $W_{i}, i=1, \ldots, n$, be irreducible complex varieties each of dimension $d$, and let $V \subset \prod_{i=1}^{n} W_{i}$ be an irreducible subvariety.

Now let $C, \tau \in \mathbb{N}$ and consider finite subsets $X_{i} \subset W_{i}$ with $\left|X_{i}\right| \leq N^{d}, N \in \mathbb{N}$, and with each $X_{i}$ in coarse $(C, \tau)$-general position in $W_{i}$. As a straightforward consequence of coarse general position, if $\tau>d$ and $C$ is sufficiently large depending on $V$ only, we will see in Lemma 7.1 that we have a trivial bound

$$
\left|V \cap \prod_{i=1}^{n} X_{i}\right| \leq O_{V}\left(N^{\operatorname{dim}(V)}\right) .
$$

We say that $V \subset \prod_{i} W_{i}$ admits a power-saving $\boldsymbol{b y} \epsilon>0$ if for some $C, \tau \in \mathbb{N}$ depending on $V$ only, this bound can be improved to $\left|V \cap \prod_{i=1}^{n} X_{i}\right| \leq O_{V, \epsilon}\left(N^{\operatorname{dim}(V)-\epsilon}\right)$. We say $V$ admits no power-saving if it does not admit a power-saving by $\epsilon$ for any $\epsilon>0$.

It is easy to see that if $V$ admits no power-saving, then $\operatorname{dim}(V)$ must be an integral multiple of $d$ (see Lemma 7.1). In Theorem 1.11 below we give a complete classification of the varieties with no power-saving. To this end we introduce as earlier a notion of special varieties, which generalises the previous definition and is slightly more involved.

Let $G$ be a connected commutative complex algebraic group, and let $\operatorname{End}(G)$ be the ring of algebraic endomorphisms of $G$. We will denote by $\operatorname{End}^{0}(G)$ the $\mathbb{Q}$ algebra $\operatorname{End}^{0}(G):=\mathbb{Q} \otimes_{\mathbb{Z}} \operatorname{End}(G)$. For example, if $G$ is a torus $G=\mathbb{G}_{m}^{r}$, then $\operatorname{End}(G)=\operatorname{Mat}_{r}(\mathbb{Z})$ and $\operatorname{End}_{0}(G)=\operatorname{Mat}_{r}(\mathbb{Q})$, and if $G=\mathbb{G}_{a}^{r}$ is a vector group, then $\operatorname{End}(G)=\operatorname{End}^{0}(G)=\operatorname{Mat}_{r}(\mathbb{C})$. In any case $\operatorname{End}(G)$ is a subring of $\operatorname{End}^{0}(G)$.

Definition 1.8. An algebraic subgroup of $G^{n}$ is called a special subgroup if it has an " $F$-structure" for some division subring $F$ of $\operatorname{End}^{0}(G)$, by which we mean that it is the connected component of the kernel ker $A \leq G^{n}$ of a matrix $A \in \operatorname{Mat}_{n}(F \cap \operatorname{End}(G))$.

For example $F$ could be trivial and equal to $\mathbb{Q}$, in which case the corresponding special subgroups will be the connected components of subgroups defined by arbitrary linear equations with integer coefficients in the $n$ co-ordinates of $G^{n}$.

Remark 1.9. It will be convenient for us to express this condition in terms of the Lie algebra $\operatorname{Lie}(H)$ of the subgroup $H \leq G^{n}$, which is defined as the tangent space at the identity as a $\mathbb{C}$-vector space. An algebraic endomorphism $\eta \in \operatorname{End}(G)$ induces by differentiation a linear map $d \eta: \operatorname{Lie}(G) \rightarrow \operatorname{Lie}(G)$, making $\operatorname{Lie}(G)$ into an $\operatorname{End}^{0}(G)$-module. Then a subgroup $H \leq G^{n}$ is a special subgroup if and only if $\operatorname{Lie}(H)=\operatorname{Lie}(G) \otimes_{F} J \leq \operatorname{Lie}(G)^{n}$ for some division subring $F \subset \operatorname{End}^{0}(G)$ and some $F$-subspace $J \leq F^{n}$ (where we make the obvious identifications between $\operatorname{Lie}(G)^{n}$, $\operatorname{Lie}\left(G^{n}\right)$ and $\left.\operatorname{Lie}(G) \otimes_{F} F^{n}\right)$.

Definition 1.10. An irreducible closed subvariety $V \subset \prod_{i=1}^{n} W_{i}$ of a product of irreducible varieties is special if it is in co-ordinatewise correspondence with a product $\prod_{i} H_{i} \leq \prod_{i} G_{i}^{n_{i}}$ of special subgroups $H_{i}$ of powers $G_{i}^{n_{i}}$ of commutative complex algebraic groups, where $\sum_{i} n_{i}=n$.

This is consistent with Definition 1.3, because when $G$ is one-dimensional, every connected algebraic subgroup of $G^{n}$ has an $F$-structure, where $F=\operatorname{End}^{0}(G)$. See Lemma 7.16 below.

We are now in a position to state our main theorem:

Theorem 1.11. Suppose $W_{1}, \ldots, W_{n}$ are irreducible complex algebraic varieties of the same dimension. Then an irreducible subvariety $V \subset \prod_{i=1}^{n} W_{i}$ admits no powersaving if and only if it is special. 
Example 1.12. Let $G:=\left(\mathbb{C}^{\times}\right)^{4}$. Then $\operatorname{End}^{0}(G)=\mathbb{Q} \otimes_{\mathbb{Z}} \operatorname{End}(G) \cong \mathbb{Q} \otimes_{\mathbb{Z}} \operatorname{Mat}_{4}(\mathbb{Z}) \cong$ $\operatorname{Mat}_{4}(\mathbb{Q})$, the ring of $4 \times 4$ rational matrices. This is certainly not a division ring, but for example the quaternion algebra

$$
\mathcal{H}_{\mathbb{Q}}=\left(\mathbb{Q}[i, j, k]: i^{2}=j^{2}=k^{2}=-1 ; i j=k ; j k=i ; k i=j\right)
$$

embeds in $\operatorname{Mat}_{4}(\mathbb{Q})$ via the left multiplication representation. This defines in particular an action of $\mathcal{H}_{\mathbb{Z}}=\mathbb{Z}[i, j, k] \subset \mathcal{H}_{\mathbb{Q}}$ on $G$ by endomorphisms given by a ring homomorphism $\alpha: \mathcal{H}_{\mathbb{Z}} \rightarrow \operatorname{End}(G), x \mapsto \alpha_{x}$ defined by:

$$
\begin{aligned}
\alpha_{i}(a, b, c, d) & =\left(b^{-1}, a, d^{-1}, c\right) ; \\
\alpha_{j}(a, b, c, d) & =\left(c^{-1}, d, a, b^{-1}\right) ; \\
\alpha_{k}(a, b, c, d) & =\left(d^{-1}, c^{-1}, b, a\right) .
\end{aligned}
$$

Then for instance $V:=\left\{\left(x, y, z_{1}, z_{2}, z_{3}\right) \in G^{5}: z_{1}=x \cdot y, z_{2}=x \cdot \alpha_{i}(y), z_{3}=\right.$ $\left.x \cdot \alpha_{j}(y)\right\}$ is a special subgroup of $G^{5}$.

To see that $V$ admits no power-saving, we can consider "approximate $\mathcal{H}_{\mathbb{Z}^{-}}$ submodules": let $H_{N}:=\{n+m i+p j+q k: n, m, p, q \in\{-N, \ldots, N\}\}$, let $g \in G$ be generic (i.e. $\mathbb{Q}(g)$ has transcendence degree 4 ), and let $X_{N}:=\alpha_{H_{N}}(g)=\left\{\alpha_{h}(g)\right.$ : $\left.h \in H_{N}\right\} \subset G$. Since $\alpha_{\mathcal{H}_{\mathbb{Z}}}(g)$ is a finitely generated subgroup of $G$, one can show (it is a consequence of Laurent's Mordell-Lang theorem for tori, see Remark 7.14) that for $W \subsetneq G$ a proper Zariski-closed subvariety, $\left|W \cap \alpha_{\mathcal{H}_{\mathbb{Z}}}(g)\right|$ is finite and bounded by a function of the complexity of $W$. Hence for all $\tau$, for all sufficiently large $N$, we have that $X_{N}$ is $\tau$-cgp in $G$. But $\alpha_{i}\left(X_{N}\right)=X_{N}=\alpha_{j}\left(X_{N}\right)$, and so $\left|X_{N}^{5} \cap V\right| \geq \Omega\left(\left|X_{N}\right|^{2}\right)$.

We show in Subsection 7.1 that any special subgroup admits no power-saving. The argument of this example goes through for many groups $G$ (see Remark 7.14), but extra complications arise with other groups - in particular in the case of a power of the additive group $G=\mathbb{G}_{a}(\mathbb{C})^{d}=\left(\mathbb{C}^{d},+\right)$, where many more division rings can arise and no Mordell-Lang type result holds.

Remark 1.13. In the situation of [ES12, Theorem 27] that $V \subset \mathbb{C}^{d} \times \mathbb{C}^{d} \times \mathbb{C}^{d}$ projects dominantly with generically finite fibres to each pair of co-ordinates, if $V$ is special then it is in co-ordinatewise correspondence with the special subgroup $H_{0}:=\left\{x_{1}+x_{2}+x_{3}=0\right\}$ of a $d$-dimensional commutative algebraic group $G$. Indeed, we first obtain from Theorem 1.11 that it is in co-ordinatewise correspondence with the connected component of $\left\{\alpha_{1} y_{1}+\alpha_{2} y_{2}+\alpha_{3} y_{3}=0\right\}$ for some self-isogenies $\alpha_{i} \in \operatorname{End}(G)$; then, setting $x_{i}:=\alpha_{i} y_{i}$, we see that this is in co-ordinatewise correspondence with $H_{0}$.

Similarly for general $n$, if $\operatorname{dim}(V)=(n-1) d$ (as in [RSdZ18] for instance) then $\left\{\sum_{i} x_{i}=0\right\}$ is the only kind of special subgroup which needs to be considered. But if $V$ has higher codimension, endomorphisms are indispensable.

Remark 1.14 (Explicit power-saving). We can consider strengthening Theorem 1.11 by replacing the condition that $V$ admits no power-saving with the condition that $V$ does not admit a power-saving by $\eta$, where the "gap" $\eta$ is a constant $\eta=\eta(d, n)>0$.

The existence of such a gap in the case $n=3$ is part of [ES12, Main Theorem], and for $n=3$ and $d=1$ an explicit value of $\eta=\frac{1}{6}$ for this gap was found independently by Wang [Wan14] and Raz, Sharir, and de Zeeuw [RSDZ16]; furthermore, [RSdZ18] finds a gap of $\eta=\frac{1}{3}$ for the case of $n=4$ and $d=1$ (under a non-degeneracy assumption). For $n=3$ and $d$ arbitrary some explicit gaps were obtained by Wang and the second author (see [BW16]). None of the gaps are known to be optimal.

Our techniques for the general situation go via the abstraction of combinatorial geometries and are not adapted to even proving the existence of a gap, still less 
calculating one. However, in Section 3 we work out the case of $n=3$, which does not require the full power of this abstraction, and we obtain there an explicit gap $\eta=\frac{1}{16}$ for all $d$ (see Theorem 3.8 below) and also recover the above-mentioned $\frac{1}{6}$ gap when $n=3$ and $d=1$.

We draw as a corollary of Theorem 1.4 the following generalised sum-product phenomenon.

Corollary 1.15 (Generalised sum-product phenomenon). Let $\left(G_{1},+_{1}\right)$ and $\left(G_{2},+_{2}\right)$ be one-dimensional non-isogenous connected complex algebraic groups, and for $i=$ 1,2 let $f_{i}: G_{i}(\mathbb{C}) \rightarrow \mathbb{C}$ be a rational map. Then there are $\epsilon, c>0$ such that if $A \subset \mathbb{C}$ is a finite set lying in the range of each $f_{i}$, then setting $A_{i}=f_{i}^{-1}(A) \subset G_{i}(\mathbb{C})$ we have

$$
\max \left(\left|A_{1}+{ }_{1} A_{1}\right|,\left|A_{2}+{ }_{2} A_{2}\right|\right) \geq c|A|^{1+\epsilon} .
$$

Remark 1.16. The usual sum-product phenomenon is the case $\left(G_{1},+_{1}\right)=(\mathbb{C},+)$ and $\left(G_{2},+_{2}\right)=(\mathbb{C} \backslash\{0\}, \cdot)$, with $f_{1}$ and $f_{2}$ being the identity maps.

If instead $G_{2}=E \subset \mathbb{P}^{2}(\mathbb{C})$ is an elliptic curve defined by $\left\{y^{2}=x(x-1)(x-\lambda)\right\}$, then we may take $f_{2}$ to be the rational map $[x: y: 1] \mapsto x$. This case of the additive group and an elliptic curve was previously considered for finite fields in [Shp08].

The constant $c$ (which must depend on the $f_{i}$ 's) is necessary as both $A_{i}$ 's could be finite subgroups of bounded order. We believe however that the power-saving $\epsilon>0$ above is uniform over all group laws (and also independent of the $f_{i}$ 's); proving this would require establishing an explicit gap in Theorem 1.4 for $d=1$ and $n=6$. We do not tackle this issue here.

We also obtain the following result on intersections of subvarieties with powers of an approximate subgroup, or just of a set with small doubling.

Theorem 1.17. Let $G$ be a commutative complex algebraic group. Suppose $V$ is a subvariety of $G^{n}$ which is not a coset of a subgroup. Then there are $N, \tau, \epsilon, \eta>0$ depending only on $G$ and the complexity of $V$ such that if $A \subset G$ is a finite subset such that $A-A$ is $\tau$-cgp and $|A+A| \leq|A|^{1+\epsilon}$ and $|A| \geq N$, then $\left|A^{n} \cap V\right|<$ $|A|^{\frac{\operatorname{dim}(V)}{\operatorname{dim}(G)}-\eta}$.

Note that Theorem 1.11 yields right away that if no such $\eta>0$ exists, then $V$ must be special. So the point here is to show that under the small doubling assumption the special $V$ 's are in fact cosets of algebraic subgroups. The result is reminiscent of the Larsen-Pink type estimates for approximate groups (see [HW08] [Hru12, Prop. 5.5], [BGT11, Thm 4.1.]), with a stronger conclusion (the powersaving $\eta>0$ ) and stronger hypothesis (coarse general position). This conclusion is also reminiscent of results in Diophantine geometry of Manin-Mumford or MordellLang type, although our methods are completely unrelated; see Example 8.3 for further comments in this direction.

1.1. Method of proof. The proof of our main results, Theorems 1.4 and 1.11, relies on an initial ultraproduct construction starting from a sequence of finite subsets witnessing the absence of power-saving. This yields pseudo-finite cartesian products. The field-theoretic algebraic closure relation then induces an abstract projective geometry at the level of the ultraproduct and we show, as a consequence of known incidence bounds generalising the Szemerédi-Trotter theorem, that this geometry is modular, i.e. satisfies the Veblen axiom of abstract projective geometries and can therefore be co-ordinatised. The division rings appearing in Theorem 1.11 arise that way. In the one-dimensional $(d=1)$ case, the projective geometries which embed in the geometry of algebraic closure in an algebraically closed field 
were characterised in [EH91], and in an appendix we use similar techniques (primarily the abelian group configuration theorem) to characterise them in the higher dimensional case. The main combinatorial results above then follow.

Much of the strategy is an implementation of ideas due to Hrushovski appearing in [Hru13], where he introduced the formalism of coarse pseudo-finite dimension and outlined a proof of the original Elekes-Szabó theorem in those terms.

More generally, our results are a consequence of specialising ideas of model theory to this combinatorial setting. We use the conventions and language of model theory throughout. Nonetheless, our treatment requires very little model-theoretic background and everything we need is described and recalled in Section 2. It is also mostly self-contained, except for the use of the group configuration theorem, recalled in Section 3, and the Szemerédi-Trotter type incidence bounds recalled in $\S 2.2$.

1.2. Related work. We remark here on how this paper relates to other recent works on applications of model theory to similar problems.

In an unreleased work in progress, Hrushovski, Bukh, and Tsimmerman consider expansion phenomena in pseudo-finite subsets of pseudo-finite fields of size comparable to that of the non-standard prime field. This context is quite different from that we consider, in particular because of the failure of Szemerédi-Trotter in this regime, but there may be some overlap in techniques; in particular, their analysis also proceeds via modularity and the abelian group configuration theorem.

Meanwhile, Chernikov and Starchenko [CS] recently proved a version of Theorem 1.2 in strongly minimal structures which are reducts of distal structures. This direction of generalisation is orthogonal to the one we consider here, where we restrict to the case of $A C F_{0}$ (this restriction is used in Lemma 2.15 and in Proposition 7.10).

1.3. Organisation of the paper. In Section 2 we set up our notation for the rest of the paper and present Hrushovski's notion of pseudo-finite dimension of internal sets and its basic properties. This section is entirely self-contained. We also recall the Szemerédi-Trotter-type bounds for arbitrary varieties and recast them in this language. In Section 3 we reprove the original Elekes-Szabó theorem using the group configuration theorem and the formalism of pseudo-finite dimensions. In higher dimensions we also recover the commutativity of the ambient group and obtain an explicit power saving of $\frac{1}{16}$. This section is not used in the proof of our main theorem, but can be read as an example of the method, worked out in a special case. In Section 4 we give a counter-example to the original Elekes-Szabó theorem when the assumption of general position is removed. Section 5 contains the proof of the key point: the modularity of the projective geometry associated to a variety without power-saving. In Sections 6 and 7 we complete the proof of Theorems 1.4 and 1.11 modulo the result proven in the Appendix. In particular we prove the converse (the "if" direction of the theorems), which requires some information regarding division subrings of matrices. We also derive Corollary 1.15. In Section 8 we prove Theorem 1.17 and draw some connections with Diophantine geometry. Finally the appendix is devoted to the higher-dimensional version of [EH91].

1.4. Acknowledgements. Thanks to Mohammad Bardestani, Elisabeth Bouscaren, Ben Green, Martin Hils, Udi Hrushovski, Jonathan Kirby, Oriol Serra, Pierre Simon and Hong Wang for helpful conversations.

We would also like to thank the Institut Henri Poincaré and the organisers of the trimester "Model theory, combinatorics and valued fields", where some of the work was done. The second author acknowledges support from ERC grant GeTeMo no. 617129 . 


\section{Contents}

1. Introduction 1

2. The non-standard setup 7

3. Warm-up: the Elekes-Szabó theorem 17

4. Necessity of general position 26

5. Projective geometries arising from varieties without power-saving $\quad 27$

6. Varieties with coherent generics 33

7. Asymptotic consequences 34

8. Coherence in subgroups 40

Appendix A. Projective geometries fully embedded in algebraic geometry 42

References 47

\section{THE NON-STANDARD SETUP}

In this preliminary section, we set up our notation and introduce the key concepts, which will be used in the proof of the main results. We assume some familiarity with the notions of first order languages, formulas and ultraproducts as expounded for example in the first two chapters of [Mar02]. No other more sophisticated model-theoretical concepts will be assumed.

2.1. Coarse pseudo-finite dimension. We begin with a self-contained presentation of Hrushovski's formalism of coarse pseudo-finite dimensions from [Hru13], slightly adapted to our purposes.

2.1.1. Ultraproducts and internal sets. We will fix a non-principal ultrafilter $\mathcal{U}$ on the set of natural numbers. We say that a property of natural numbers holds for $\mathcal{U}$-almost every $s$ if the set of natural numbers $s$ for which the property holds is an element of $\mathcal{U}$.

We form the ultraproduct $K=\prod_{s \rightarrow \mathcal{U}} K_{s}$ of countably many algebraically closed fields $K_{s}, s \geq 0$, which by definition is the cartesian product $\prod_{s>0} K_{s}$ quotiented by the equivalence relation $\left(x_{s}\right)_{s} \sim\left(y_{s}\right)_{s}$ if and only if $x_{s}=y_{s}$ for $\mathcal{U}$-almost every $s \geq 0$. The field $K$ is also algebraically closed.

We will assume throughout internal characteristic zero; namely, we assume $\operatorname{char}\left(K_{s}\right)=0$ for all $s$. This is required for the incidence bounds used in Lemma 2.15 below. (See [Hru13, Corollary 5.6] for discussion on how it ought to be possible to weaken this assumption).

In fact for our purposes it makes no difference to simply make the following

Assumption 2.1. We assume that $K_{s}=\mathbb{C}$ for all $s$.

We denote by ${ }^{*} \mathbb{R}:=\mathbb{R}^{\mathcal{U}}$ the corresponding ultrapower of $\mathbb{R}$, and call its elements non-standard reals. The real field $\mathbb{R}$ embeds diagonally in ${ }^{*} \mathbb{R}$ and its elements are called standard reals. The order on $\mathbb{R}$ extends to an order on ${ }^{*} \mathbb{R}$ by saying that $x<y$ if and only if $x_{s}<y_{s}$ for $\mathcal{U}$-almost every $s \geq 0$.

We let $s t:{ }^{*} \mathbb{R} \rightarrow \mathbb{R} \cup\{-\infty, \infty\}$ be the standard part map, namely $\operatorname{st}(\xi)$ is $\infty$ (resp. $-\infty$ ) if $\xi=\left(\xi_{s}\right)_{s>0} \in{ }^{*} \mathbb{R}$ is larger (resp. smaller) than any standard real, and otherwise it is the ultralimit along $\mathcal{U}$ of the sequence $\left(\xi_{s}\right)_{s}$, namely the unique $z \in \mathbb{R}$ such that for each $\epsilon>0,\left|z-\xi_{s}\right|<\epsilon$ holds for $\mathcal{U}$-almost every $s$.

Let $n$ be a positive integer. We say that a subset $X \subset K^{n}$ is internal if $X=\prod_{s \rightarrow \mathcal{U}} X^{K_{s}}$ for some subsets $X^{K_{s}} \subset K_{s}^{n}$.

2.1.2. Saturation and compactness. A standard property of ultraproducts over a countable index set is their $\aleph_{1}$-compactness. Namely countable families of internal 
sets have the finite intersection property. This means that for each positive integer $n$, if $X_{0} \supset X_{1} \supset \ldots$ is a countable chain of internal subsets of $K^{n}$ such that $\bigcap_{i \geq 0} X_{i}=\emptyset$, then $X_{i}=\emptyset$ for some $i \geq 0$. Equivalently if an internal set $X \subset K^{n}$ lies in the union of countably many internal sets, then it already lies in the union of finitely many of them.

2.1.3. Coarse pseudo-finite dimension. Throughout we will fix once and for all some infinite non-standard real $\xi \in{ }^{*} \mathbb{R}$ with $\xi>\mathbb{R}$, which we call the scaling constant. This choice corresponds to a choice of calibration for the large finite sets involved in our main results. Given an internal set $X=\prod_{s \rightarrow \mathcal{U}} X^{K_{s}} \subset K^{n}$, we define the non-standard cardinality of $X$ by $|X|:=\prod_{s \rightarrow \mathcal{U}}\left|X^{K_{s}}\right| \in{ }^{*} \mathbb{R} \cup\{\infty\}$ and its coarse pseudo-finite dimension $\delta(X)$ by

$$
\boldsymbol{\delta}(X):=\operatorname{st}\left(\frac{\log |X|}{\log \xi}\right) \in \mathbb{R}_{\geq 0} \cup\{-\infty, \infty\}
$$

(for the empty set we adopt the convention $\boldsymbol{\delta}(\emptyset)=\log (0)=-\infty$ ).

Example 2.2. Let $X^{K_{s}}:=\left\{(p, q) \in \mathbb{N}^{2}: p+q<s\right\}, Y^{K_{s}}:=\left\{1, \ldots, s^{s}\right\}$ and $\xi_{s}:=s$ for all $s \geq 1$. Then $\boldsymbol{\delta}(X)=2$ and $\boldsymbol{\delta}(Y)=\infty$.

We note here the following immediate properties of the coarse dimension, for internal sets $A, B \subset K^{n}$ :

(1) (non-negativity) $\boldsymbol{\delta}(A) \geq 0$ if $A$ is non-empty,

(2) (monotonicity) If $A \subset B$, then $\boldsymbol{\delta}(A) \leq \boldsymbol{\delta}(B)$,

(3) (ultrametricity) $\boldsymbol{\delta}(A \cup B)=\max \{\boldsymbol{\delta}(A), \boldsymbol{\delta}(B)\}$.

2.1.4. Definable sets. In order to talk about definable subsets of $K^{n}$ we fix a language $\mathcal{L}$, which extends the language of rings $\mathcal{L}_{\text {ring }}=(+,-, \cdot, 0,1)$ by only countably many symbols. We assume each $K_{s}$ is an $\mathcal{L}$-structure, and equip the ultraproduct $K$ with the corresponding $\mathcal{L}$-structure. If $C \subset K$ is a countable set, we write $\mathcal{L}_{C}$ for the language with new constant symbols for the elements of $C$.

To every first order formula $\phi=\phi(\bar{x})$ in the language $\mathcal{L}_{C}$ with free variables $\bar{x}:=\left(x_{1}, \ldots, x_{n}\right)$ there corresponds a definable set $\phi(K):=\left\{\bar{k} \in K^{n}\right.$ : $\phi(\bar{k})$ holds in $K\}$. We say that the set $\phi(K)$ is $C$-definable or definable over $C$; in other words, $\phi(K)$ is definable by a formula with parameters from $C$. When $C=\emptyset$, we say that $\phi(K)$ is definable over $\emptyset$, or definable without parameters.

We set

$$
K^{<\infty}:=\bigcup_{n \geq 1} K^{n}
$$

the set of all finite tuples of elements of $K$. The family $\mathcal{D}_{n, C}$ of $C$-definable subsets of $K^{n}$ forms a boolean algebra, which contains all algebraic sets defined over $C$ (i.e. solutions of polynomial equations whose coefficients are elements of $C$ ) as well as a countable number of prescribed subsets (the graphs of functions from $\mathcal{L}$ and sets of tuples satisfying the relations whose symbols belong to $\mathcal{L}$ ) and $\cup_{n \geq 1} \mathcal{D}_{n, C}$ is stable under co-ordinate projections (image and pre-image). Equivalently, instead of starting with the language $\mathcal{L}$ and considering the associated definable sets, we may begin by giving ourselves for each $n$ a countable number of prescribed internal subsets of $K^{n}$ and consider the smallest family of subsets of $K^{<\infty}$ which contains them as well as all algebraic sets defined over $C$ and is stable under union, complement and co-ordinate projections (image and pre-image).

Clearly every definable set is internal. The converse is not true, however any single internal set in $K^{n}$ (or a countable family of such) can be made definable by expanding the language by adding an $n$-ary relation symbol for that internal set. This will be done below in order to make $\boldsymbol{\delta}$ continuous and further down in the 
paper when, in our combinatorial applications, we will always add the ultraproduct of the finite sets $X_{i}$ to the class of definable sets.

Remark 2.3 (Notation for tuples). We will often write a couple $(a, b) \in K^{2}$ as $a b$, or given two tuples $\bar{a} \in K^{n}$ and $\bar{b} \in K^{m}$, we will denote the tuple $(\bar{a}, \bar{b}) \in K^{n+m}$ simply by $\bar{a} \bar{b}$, concatenating the two tuples.

2.1.5. Types, $\bigwedge$-definable sets and coarse dimension of a tuple. The type $\operatorname{tp}(\bar{a})$ of a tuple $\bar{a} \in K^{n}$ is the family of all formulae in $n$ variables in the language $\mathcal{L}$ (that is, without parameters) satisfied by $\bar{a}$. The intersection of all $\emptyset$-definable subsets containing $\bar{a}$ will be denoted by $\operatorname{tp}(\bar{a})(K)$. Similarly if $C$ is a countable subset of $K$, we denote by $\operatorname{tp}(\bar{a} / C)$ the (countable) family of all formulae in $\mathcal{L}_{C}$ with $n$ variables satisfied by $\bar{a}$ and we set:

$$
\operatorname{tp}(\bar{a} / C)(K):=\bigcap_{\phi \in \operatorname{tp}(\bar{a} / C)} \phi(K)=\bigcap_{\bar{a} \in Y, Y} Y .
$$

By a $\bigwedge$-definable set over $C$ (say "type-definable", or "wedge-definable"), we mean a subset $X$ of $K^{n}$, for some $n$, which is the intersection of countably many $C$-definable sets. Such sets need not be internal. We say a set is $\Lambda$-internal if it is the intersection of a countable collection of internal sets; so $\bigwedge$-definable sets are $\bigwedge$-internal.

For a $\bigwedge$-internal set $X \subset K^{n}$, we define

$$
\boldsymbol{\delta}(X):=\inf \{\boldsymbol{\delta}(Y): Y \supset X, Y \text { internal }\} .
$$

It is an immediate consequence of $\aleph_{1}$-compactness that if $X \subset K^{n}$ is the intersection of a countable decreasing chain $X_{0} \supset X_{1} \supset \ldots$ of internal subsets of $K^{n}$ then $\boldsymbol{\delta}(X)=\inf _{i} \boldsymbol{\delta}\left(X_{i}\right)$. In particular if $X \subset K^{n}$ is a $\bigwedge$-definable set over a countable set $C \subset K$, then

$$
\boldsymbol{\delta}(X)=\inf \{\boldsymbol{\delta}(Y): Y \supset X, Y \text { definable over } C\} .
$$

The set $\operatorname{tp}(\bar{a} / C)(K)$ is $\bigwedge$-definable, so this allows to define $\boldsymbol{\delta}(\bar{a} / C)$, the coarse dimension of the tuple $\bar{a}$ over $C$, as $\boldsymbol{\delta}(\operatorname{tp}(\bar{a} / C)(K))$. Namely for $\bar{a} \in K^{n}$ :

$$
\boldsymbol{\delta}(\bar{a} / C)=\inf \left\{\boldsymbol{\delta}(Y): \bar{a} \in Y \subset K^{n}, Y \text { definable over } C\right\} .
$$

Abusing notation we will write $\boldsymbol{\delta}(\bar{a})$ for $\boldsymbol{\delta}(\bar{a} / \emptyset)$, and similarly if $C \subset K^{<\infty}$ (as opposed to just $K)$ we will denote by $\operatorname{tp}(\bar{a} / C)$ and $\delta(\bar{a} / C)$ the type and coarse dimension of $\bar{a}$ over the subset $C^{\prime} \subset K$ of all co-ordinates of tuples from $C$. Further note that if $C_{1} \subset C_{2} \subset K^{<\infty}$, then

$$
\boldsymbol{\delta}\left(\bar{a} / C_{2}\right) \leq \boldsymbol{\delta}\left(\bar{a} / C_{1}\right) .
$$

Given a $\bigwedge$-definable set $X \subset K^{n}$ over a countable set $C \subset K$, we clearly have $\boldsymbol{\delta}(\bar{a} / C) \leq \boldsymbol{\delta}(X)$ for every $\bar{a} \in X$. An important consequence of $\aleph_{1}$-compactness, which will be used several times in the proofs, is the existence of some tuple realising the dimension:

Fact 2.4 ("existence of an independent realisation"). If $X \subset K^{n}$ is a $\bigwedge$-definable set over a countable set $C \subset K$, then $X$ contains some $\bar{a} \in X$ with

$$
\boldsymbol{\delta}(\bar{a} / C)=\boldsymbol{\delta}(X) .
$$

It is for this that we require countability of the language.

Proof. Note that for any $\bar{a} \in X$, we have $\boldsymbol{\delta}(\bar{a} / C)<\boldsymbol{\delta}(X)$ if and only if there is a $C$-definable subset $Z \subset K^{n}$ such that $\bar{a} \in Z$ and $\delta(Z)<\delta(X)$. Consider the family of all $C$-definable subsets $Z$ with $\delta(Z)<\delta(X)$. It is enough to show that their union does not contain $X$. But if this were the case, by $\aleph_{1}$-compactness (see 2.1.2), $X$ would be contained in the union of finitely many of them, say $Z_{1}, \ldots, Z_{m}$. 
So $\boldsymbol{\delta}\left(\bigcup_{1}^{m} Z_{i}\right) \geq \boldsymbol{\delta}(X)$ by monotonicity. However $\boldsymbol{\delta}\left(\bigcup_{1}^{m} Z_{i}\right)=\max \boldsymbol{\delta}\left(Z_{i}\right)$ by the ultrametricity property (see 2.1.3 above), and hence is $<\delta(X)$, a contradiction.

Finally we record the following straightforward observation:

Fact 2.5. For a tuple $\bar{a}=\left(a_{1}, \ldots, a_{n}\right) \in K^{n}$ and a countable set $C \subset K$, the coarse dimension $\boldsymbol{\delta}(\bar{a} / C)$ depends only on the set of co-ordinates $\left\{a_{1}, \ldots, a_{n}\right\} \subset K$.

Proof. Indeed it is invariant under any permutation of the co-ordinates, because these induce bijections of $K^{n}$ and thus preserve cardinality. And furthermore if $X$ is an internal set in $K^{n}$ such that the last two co-ordinates $x_{n-1}$ and $x_{n}$ coincide for all $x \in X$, then $\boldsymbol{\delta}(X)=\boldsymbol{\delta}(\pi(X))$, where $\pi(X)$ is the projection to the first $n-1$ co-ordinates.

2.1.6. Continuity, additivity and invariance of coarse dimension. We now come to two crucial properties of $\boldsymbol{\delta}$ : its additivity and its invariance. This will turn $\boldsymbol{\delta}$ into a dimension-like quantity with properties very similar to those say of the transcendence degree of the field extension generated by a tuple. To get these properties it is enough to prove that $\boldsymbol{\delta}$ has the continuity property we will now define. This property essentially amounts to requiring that for each definable set the subset of fibers of a given size under a co-ordinate projection is itself a definable set or is at least well approximated by one. However continuity is not automatic and to get it we will need to enrich our language $\mathcal{L}$ somewhat artificially, by adding a (still countable) family of definable subsets.

Definition 2.6. We say that $\boldsymbol{\delta}$ has the continuity property (or is continuous) if given $n, m \geq 1, \alpha \in \mathbb{R}, \epsilon>0$ and a $\emptyset$-definable set $Y \subset K^{n} \times K^{m}$ there is a $\emptyset$-definable set $W \subset K^{m}$ such that

$$
\left\{\bar{b} \in K^{m}: \boldsymbol{\delta}\left(Y_{\bar{b}}\right) \geq \alpha+\epsilon\right\} \subset W \subset\left\{\bar{b} \in K^{m}: \boldsymbol{\delta}\left(Y_{\bar{b}}\right) \geq \alpha\right\},
$$

where $Y_{\bar{b}}$ is the fiber $\left\{\bar{x} \in K^{n} ;(\bar{x}, \bar{b}) \in Y\right\}$.

It is always possible to force the continuity of $\boldsymbol{\delta}$ by enlarging the language $\mathcal{L}$ to a new language $\mathcal{L}^{\prime}$, which is still countable and for which $\boldsymbol{\delta}$ becomes continuous. Indeed for each $q \in \mathbb{Q}$ we may add a predicate to simulate the quantifier $\exists_{\geq \xi^{q}}$ of having "at least $\xi^{q}$ solutions". Explicitly, if $\xi=\lim _{s \rightarrow \mathcal{U}} \xi_{s}$ is as in the definition of $\boldsymbol{\delta}$, let $\mathcal{L}_{0}:=\mathcal{L}$ and define $\mathcal{L}_{i+1}$ by adding to $\mathcal{L}_{i}$ a new predicate $\psi_{\phi(\bar{x}, \bar{y}), q}(\bar{y})$ for each formula $\phi(\bar{x}, \bar{y}) \in \mathcal{L}_{i}$ and each $q \in \mathbb{Q}$, interpreted in $K_{s}$ by

$$
\psi_{\phi(\bar{x}, \bar{y}), q}\left(K_{s}\right):=\left\{\bar{y}:\left|\phi\left(K_{s}, \bar{y}\right)\right| \geq \xi_{s}^{q}\right\}
$$

where we have written $\phi\left(K_{s}, \bar{y}\right)$ for $\left\{\bar{x}: \phi(\bar{x}, \bar{y})\right.$ holds in $\left.K_{s}\right\}$. So in the ultraproduct $K$ we have $\psi_{\phi(\bar{x}, \bar{y}), q}(K)=\left\{\bar{y}:|\phi(K, \bar{y})| \geq \xi^{q}\right\}$. Then we set $\mathcal{L}^{\prime}:=\cup_{i<\infty} \mathcal{L}_{i}$.

It is then clear that $\delta$ is continuous once we replace $\mathcal{L}$ with $\mathcal{L}^{\prime}$. Indeed if $\alpha \in \mathbb{R}$ and $\epsilon>0$ we may pick a rational $q \in(\alpha, \alpha+\epsilon)$. Then if $\bar{b} \in \psi_{\phi(\bar{x}, \bar{y}), q}(K)$ then $|\phi(K, \bar{b})| \geq \xi^{q}$ so $\boldsymbol{\delta}(\phi(K, \bar{b})) \geq q>\alpha$, while if $\boldsymbol{\delta}(\phi(K, \bar{b})) \geq \alpha+\epsilon$ then $\boldsymbol{\delta}(\phi(K, \bar{b})) \geq q$ and $\bar{b} \in \psi_{\phi(\bar{x}, \bar{y}), q}(K)$.

Remark 2.7. Note that the continuity property automatically extends to definable sets with parameters. Namely if $Y$ is assumed $C$-definable for some $C \subset K$, and $\boldsymbol{\delta}$ is continuous, we may find a $C$-definable $W$ as in Definition 2.6. Indeed there is a finite tuple $\bar{c}_{0} \in K^{\ell}$ for some $\ell \geq 1$ with co-ordinates in $C$ such that $Y=Y_{\bar{c}_{0}}^{0}=$ $\left\{(\bar{x}, \bar{y}):\left(\bar{x}, \bar{y}, \bar{c}_{0}\right) \in Y^{0}\right\}$ for some $\emptyset$-definable set $Y^{0} \in K^{n+m+\ell}$, so by continuity there is $W^{0}$ a $\emptyset$-definable subset of $K^{m}$ such that

$$
\left\{(\bar{b}, \bar{c}) \in K^{m+\ell}: \boldsymbol{\delta}\left(Y_{\bar{b}, \bar{c}}\right) \leq \alpha\right\} \subset W^{0} \subset\left\{(\bar{b}, \bar{c}) \in K^{m+\ell}: \boldsymbol{\delta}\left(Y_{\bar{b}, \bar{c}}\right) \leq \alpha+\epsilon\right\} .
$$

But now $W:=W_{\bar{c}_{0}}^{0}$ is the desired $C$-definable set. 
Continuity yields the following crucial properties, which are characteristic of a dimension function; in particular, they are shared by transcendence degree.

Fact 2.8. Let $\bar{a}, \bar{b} \in K^{<\infty}$ and let $C \subset K$ be countable and $\phi(\bar{x}, \bar{y})$ a formula in the language $\mathcal{L}$. If $\boldsymbol{\delta}$ is continuous (for $\mathcal{L}$ ) then it is

(i) invariant: if $\operatorname{tp}(\bar{a})=\operatorname{tp}(\bar{b})$, then $\boldsymbol{\delta}(\phi(K, \bar{a}))=\boldsymbol{\delta}(\phi(K, \bar{b}))$,

(ii) additive:

$$
\boldsymbol{\delta}(\bar{a} \bar{b} / C)=\boldsymbol{\delta}(\bar{b} / C)+\boldsymbol{\delta}(\bar{a} / \bar{b} C) .
$$

Here as above $\phi(K, \bar{a})$ denotes the definable set $\{\bar{x}: \phi(\bar{x}, \bar{a})$ holds $\}$. We have used the convention $\alpha+\infty=\infty+\alpha=\infty$, and $\bar{a} \bar{b}$ is a shorthand for $(\bar{a}, \bar{b})$, the concatenation of the tuples $\bar{a}$ and $\bar{b}$. Also we wrote $\bar{b} C$ for the union of $C$ and the co-ordinates of $\bar{b}$.

Proof. When $\bar{a}$ and $\bar{b}$ have the same type they belong to the same definable sets, so $(i)$ is immediate from the continuity of $\boldsymbol{\delta}$. The proof of $(i i)$ is given in [Hru13, Lemma 2.10]. We give it again here for the reader's convenience. The idea is the following: if $Y$ is a $C$-definable set in $K^{n} \times K^{m}$ containing $(\bar{a}, \bar{b})$ and such that all fibers $Y_{\bar{b}^{\prime}}=Y \cap \pi_{2}^{-1}\left(\bar{b}^{\prime}\right)$ above the points $\bar{b}^{\prime} \in \pi_{2}(Y)$ (where $\pi_{2}$ is the co-ordinate projection to $\left.K^{m}\right)$ have the same size, then clearly $\boldsymbol{\delta}(Y)=\boldsymbol{\delta}\left(\pi_{2}(Y)\right)+\boldsymbol{\delta}\left(Y_{\bar{b}^{\prime}}\right)$. Now the continuity property of $\boldsymbol{\delta}$ ensures that we can find a $Y$ with $\boldsymbol{\delta}(Y)$ close to $\boldsymbol{\delta}(\bar{a} \bar{b} / C)$ and with all fibers of almost the same size. This shows additivity.

We now give more details: by definition of the coarse dimension as an infimum (see (3)), given $\epsilon>0$ we may find $C$-definable sets $Y, Y^{\prime} \subset K^{n} \times K^{m}$ such that $\bar{a} \bar{b} \in Y, Y^{\prime}$ and $\boldsymbol{\delta}(\bar{a} \bar{b} / C) \leq \boldsymbol{\delta}(Y) \leq \boldsymbol{\delta}(\bar{a} \bar{b} / C)+\epsilon, \boldsymbol{\delta}(\bar{a} / \bar{b} C) \leq \boldsymbol{\delta}\left(Y_{\bar{b}}^{\prime}\right) \leq \boldsymbol{\delta}(\bar{a} / \bar{b} C)+\epsilon$ and a $C$-definable set $Z \subset K^{m}$ with $\bar{b} \in Z$ and $\boldsymbol{\delta}(\bar{b} / C) \leq \boldsymbol{\delta}(Z) \leq \boldsymbol{\delta}(\bar{b} / C)+\epsilon$. Replacing $Y, Y^{\prime}$ by $Y \cap Y^{\prime} \cap \pi_{2}^{-1}(Z)$, we may assume that $Y=Y^{\prime}$ and $Z=\pi_{2}(Y)$. Now by continuity of $\boldsymbol{\delta}$ there is a $C$-definable set $W \ni \bar{b}$ such that $\left|\boldsymbol{\delta}\left(Y_{\bar{b}^{\prime}}\right)-\boldsymbol{\delta}\left(Y_{\bar{b}}\right)\right|<\epsilon$ for all $\bar{b}^{\prime} \in W$. We may then further replace $Y$ by $Y \cap \pi_{2}^{-1}(W)$ and get to a situation where $\boldsymbol{\delta}(\bar{a} \bar{b} / C) \leq \boldsymbol{\delta}(Y) \leq \boldsymbol{\delta}(\bar{a} \bar{b} / C)+\epsilon, \boldsymbol{\delta}(\bar{b} / C) \leq \boldsymbol{\delta}\left(\pi_{2}(Y)\right) \leq \boldsymbol{\delta}(\bar{b} / C)+\epsilon$ and all fibers $Y_{\bar{b}^{\prime}}$ for $\bar{b}^{\prime} \in \pi_{2}(Y)$ have $\boldsymbol{\delta}(\bar{a} / \bar{b} C)-\epsilon \leq \boldsymbol{\delta}\left(Y_{\bar{b}^{\prime}}\right) \leq \boldsymbol{\delta}(\bar{a} / \bar{b} C)+\epsilon$. We thus conclude that $|\boldsymbol{\delta}(\bar{a} \bar{b} / C)-\boldsymbol{\delta}(\bar{b} / C)-\boldsymbol{\delta}(\bar{a} / \bar{b} C)| \leq 3 \epsilon$ as desired.

Remark 2.9. We briefly remark in passing for the model-theoretically inclined reader that a more sophisticated setup is also available, which in some ways is more satisfactory than that described above. Working directly in a countable ultrapower with only $\aleph_{1}$-compactness, as we have in this section, has the consequence that we must pick a countable language to work with. In our applications we will have no real control over the definable sets and can expect no tameness, so having to make this choice is something of a distraction. An alternative would be to define $K$ as above but in a language $\mathcal{L}_{\text {int }}$ which includes all internal sets as predicates, and then to take a $\kappa$-saturated $\kappa$-strongly homogeneous elementary extension $\mathbb{K}$, for a cardinal $\kappa$ which is larger than any parameter set we wish to consider. There is then a unique way to define $\boldsymbol{\delta}(\phi(\bar{x}, \bar{a}))$ for $\phi \in \mathcal{L}_{\text {int }}$ and $\bar{a} \in \mathbb{K}^{<\omega}$ such that $\boldsymbol{\delta}$ is continuous and extends the original definition in the case $\bar{a} \in K^{<\omega}$. Namely, $\boldsymbol{\delta}(\phi(\bar{x}, \bar{a})):=\sup \left\{q \in \mathbb{Q}: \mathbb{K} \vDash \exists_{\geq \xi^{q}} \bar{x} . \phi(\bar{x}, \bar{a})\right\}$, where $\exists_{\geq \xi^{q}} \bar{x} . \phi(\bar{x}, \bar{y})$ denotes an $\mathcal{L}_{\text {int }}$ formula with free variables $\bar{y}$ such that $K \vDash \exists_{\geq \xi^{q}} \bar{x} . \phi(\bar{x}, \bar{b})$ if and only if $|\phi(K, \bar{b})| \geq \xi^{q}$, for $\bar{b} \in K^{<\omega}$. (This is parallel to the way one defines dimension on an elementary extension of a Zariski structure.) Here, continuity is meant in the sense of Definition 2.6 - or equivalently, that the map from the type space to the 2-point compactification $S_{\bar{y}}(\emptyset) \rightarrow \mathbb{R} \cup\{-\infty, \infty\}: \operatorname{tp}(\bar{b}) \mapsto \boldsymbol{\delta}(\phi(\bar{x}, \bar{b}))$ is well-defined 
and continuous. We can then work with elements of $\mathbb{K}$ in order to analyse the internal subsets of $K$. We will not use this alternative presentation, but some readers may prefer to pretend that we do so throughout.

2.1.7. Algebraic independence and transcendence degree. At the heart of the combinatorial results of this paper lies the interplay between combinatorics (via the coarse pseudo-finite dimension $\boldsymbol{\delta}$ ) and algebraic geometry (via the notion of algebraic dimension, or transcendence degree). To this effect we will fix a base field $C_{0}$ and assume it is countable and algebraically closed and contained in $K$. We will then have to consider the subclass of definable sets that are $C_{0}$-definable using only the language of rings $\mathcal{L}_{\text {ring }}$. In the applications $C_{0}$ will be the algebraic closure of the field of definition of the variety. As is well-known, in an algebraically closed field, the sets that are $C_{0}$-definable in $\mathcal{L}_{\text {ring }}$ coincide with the so-called constructible sets of algebraic geometry defined over $C_{0}$, namely solutions of finitely many polynomial equations and inequations with coefficients in $C_{0}$. After enlarging $\mathcal{L}$ if necessary we can make the following

Assumption 2.10. We assume that $\mathcal{L}$ contains a constant symbol for each element of $C_{0}$.

Notation (0 superscript). We will use a superscript 0 , e.g. $\mathrm{tp}^{0}$, to indicate that we work in the structure $\left(K ;+, \cdot,(c)_{c \in C_{0}}\right)$ of $K$ as an algebraically closed field extension of the base field $C_{0}$, rather than in the full language $\mathcal{L}$. For example for $\bar{a}, \bar{b} \in K^{<\infty}$ and $C \subset K^{<\infty}$, saying that $\operatorname{tp}^{0}(\bar{a} / C)=\operatorname{tp}^{0}(\bar{b} / C)$ means that they satisfy the same polynomial equations over the field $C_{0}(C)$ generated by $C_{0}$ and the co-ordinates of all tuples belonging to $C$, i.e. for $f \in C_{0}(C)[\bar{X}], f(\bar{a})=0$ if and only if $f(\bar{b})=0$.

Notation (algebraic closure $\mathrm{acl}^{0}$ ). Similarly for a subset $A \subset K^{<\infty}$ we denote by $\operatorname{acl}^{0}(A)$ the field-theoretic algebraic closure in $K$ of the subfield $C_{0}(A)$ generated by $C_{0}$ and the co-ordinates of the elements of $A$.

When there is no superscript, we work in the full language $\mathcal{L}$.

Notation (transcendence degree $\mathrm{d}^{0}$ ). We write $\mathrm{d}^{0}$ for the dimension with respect to $\operatorname{acl}^{0}$, i.e. for $A, B \subset K^{<\infty}$ we set:

$$
\mathrm{d}^{0}(A / B):=\operatorname{trd}\left(C_{0}(A B) / C_{0}(B)\right),
$$

where trd denotes the transcendence degree, and $C_{0}(B)$ the field extension of $C_{0}$ generated by $B$ and $A B$ is short for $A \cup B$.

Note that, just like $\boldsymbol{\delta}, \mathrm{d}^{0}$ is additive: if $\bar{a}, \bar{b} \in K^{<\infty}$ and $C \subset K$, then

$$
\mathrm{d}^{0}(\bar{a} \bar{b} / C)=\mathrm{d}^{0}(\bar{a} / \bar{b} C)+\mathrm{d}^{0}(\bar{b} / C),
$$

where, as earlier, $\bar{b} C$ is short for the union of $C$ and the co-ordinates of $\bar{b}$.

Note finally that clearly $\mathrm{d}^{0}(A / B)=\mathrm{d}^{0}\left(A / \operatorname{acl}^{0}(B)\right)$.

Notation (independence $\downarrow_{C}^{0}$ ). If $A, B, C$ are subsets of tuples of $K$, we will say that $A$ is algebraically independent of $B$ over $C$ and write $A \downarrow_{C}^{0} B$ if

$$
\mathrm{d}^{0}(A / B C)=\mathrm{d}^{0}(A / C),
$$

i.e. if $C_{0}(A)$ is algebraically independent from $C_{0}(B)$ over $C_{0}(C)$. This is clearly a symmetric relation, namely $A \downarrow_{C}^{0} B$ if and only if $B \downarrow_{C}^{0} A$.

Notation. For $A \subset K^{<\infty}$, we write

$$
\operatorname{acl}^{0}(A)^{<\infty}:=\bigcup_{n \geq 1}\left(\operatorname{acl}^{0}(A)\right)^{n} \subset K^{<\infty},
$$


for the set of tuples algebraic over $A$. Note that this is also the set of tuples with finite orbit under the group of field automorphisms $\operatorname{Aut}\left(K / C_{0}(A)\right)$ fixing $C_{0}(A)$ pointwise.

2.1.8. Coarse dimension of an algebraic tuple. Let $C \subset K^{<\infty}$ be a countable subset. If a tuple $\bar{a}$ belongs to $\operatorname{acl}^{0}(C)^{<\infty}$, then it is contained in a finite $C$-definable set, namely the Galois orbit of $\bar{a}$ over $C_{0}(C)$. In particular, since $\xi>\mathbb{R}$ we have $\boldsymbol{\delta}(\bar{a} / C)=0$. So

$$
\bar{a} \in \operatorname{acl}^{0}(C)^{<\infty} \Rightarrow \boldsymbol{\delta}(\bar{a} / C)=0 .
$$

We also record here the following generalisation of this observation, which will be used in the proof of Proposition 5.14. For any $\bar{a} \in K^{<\infty}$ and countable $C \subset K$ :

$$
\boldsymbol{\delta}(\bar{a} / C)=\boldsymbol{\delta}\left(\bar{a} / \operatorname{acl}^{0}(C)\right) .
$$

Indeed, first we have $\boldsymbol{\delta}(\bar{a} / C) \geq \boldsymbol{\delta}\left(\bar{a} / \operatorname{acl}^{0}(C)\right)$ by (4). For the opposite inequality it is enough to show that if $\bar{b} \in \operatorname{acl}^{0}(C)$, then $\boldsymbol{\delta}(\bar{a} / C) \leq \boldsymbol{\delta}(\bar{a} / \bar{b})$. To see this, note that $\boldsymbol{\delta}(\bar{b} / C)=0$ by the above remark, and thus by additivity $(2.8 . i i)$

$$
\boldsymbol{\delta}(\bar{a} / C) \leq \boldsymbol{\delta}(\bar{a} \bar{b} / C)=\boldsymbol{\delta}(\bar{a} / \bar{b} C)+\boldsymbol{\delta}(\bar{b} / C)=\boldsymbol{\delta}(\bar{a} / \bar{b} C) \leq \boldsymbol{\delta}(\bar{a} / \bar{b}) .
$$

2.1.9. Locus of a tuple. If $\bar{a} \in K^{n}$ and $C \subset K$, we define the locus of $\bar{a}$ over $C_{0}(C)$, denoted by $\operatorname{loc}^{0}(\bar{a} / C)$, to be the smallest Zariski-closed subset $V \subset K^{n}$ such that $\bar{a} \in V$ and $V$ is defined by the vanishing of polynomials with coefficients in $C_{0}(C)$. We also write $\operatorname{loc}^{0}(\bar{a})$ for $\operatorname{loc}^{0}(\bar{a} / \emptyset)$.

Note that by definition $\operatorname{loc}^{0}(\bar{a} / C)$ is irreducible over $C_{0}(C)$, i.e. it cannot be written as a finite union of more than one proper Zariski-closed subset of $K^{n}$ defined over $C_{0}(C)$, but it may not be absolutely irreducible (i.e. irreducible over $K$ ). However each absolutely irreducible component is defined over some finite algebraic extension. In particular $\operatorname{loc}^{0}\left(\bar{a} / \operatorname{acl}^{0}(C)\right)$ is an absolutely irreducible component of $\operatorname{loc}^{0}(\bar{a} / C)$, and

$$
\mathrm{d}^{0}(\bar{a} / C)=\operatorname{dim}\left(\operatorname{loc}^{0}(\bar{a} / C)\right)=\operatorname{dim}\left(\operatorname{loc}^{0}\left(\bar{a} / \operatorname{acl}^{0}(C)\right)\right) .
$$

2.1.10. Abstract varieties. Our setup is adapted to working with tuples of elements of $K$, but in our applications we will want to work with points of algebraic groups and of general abstract algebraic varieties. We explain here how we bridge this gap using standard notions from the model theory of algebraically closed fields, as described in [Pil98] or [Mar02, 7.4].

We adopt the convention that varieties are always separated, but not necessarily irreducible.

If $V$ is an algebraic variety over an algebraically closed subfield $C \leq K$, then $V$ admits a cover by finitely many affine open subvarieties over $C$; that is, there are open subvarieties $V_{i} \subset V$ and (closed) affine subvarieties $U_{i} \subset \mathbb{A}^{n_{i}}$ and isomorphisms $f_{i}: V_{i} \rightarrow U_{i}$ over $C$, such that $V=\bigcup_{i} V_{i}$. Then $V(K)$ can be identified with the quotient of the disjoint union of the $V_{i}(K)$ by the equivalence relation of representing the same point of $V(K)$. Now $A C F_{0}$, the theory of algebraically closed fields of characteristic zero, admits elimination of imaginaries, which exactly means that such a quotient is in definable bijection over $C$ with a definable (i.e. constructible) subset of $K^{n}$ for some $n$. We refer to [Pi198, Remark 3.10(iii), Lemma 1.7] for details of this construction. In this way, we embed $V(K)$ as a subset of $K^{n}$. Note that this embedding is not continuous.

The precise embedding depends on our choice of cover. However, if $W \subset V$ is another subvariety and $f: W \rightarrow U \subset K^{m}$ is an isomorphism over $C$ with an affine variety, and if $a \in W(K) \subset V(K) \subset K^{n}$, then the subfield of $K$ generated over $C$ by the co-ordinates of $a$ according to our embedding of $V(K)$ in $K^{n}$ and those 
according to $f$ are equal, $C(a)=C(f(a))$. In particular, for $a \in V(K)$ the subfield $C(a) \leq K$ does not depend on our choice of cover.

For $\tau \in \mathbb{N}$, we say that the complexity of a closed subvariety $W \subset V$ is at most $\tau$ if for each $i$ the affine variety $f_{i}\left(W \cap V_{i}\right) \subset U_{i} \subset \mathbb{A}^{n_{i}}$ can be defined as the set of common zeros of a collection of polynomials each of degree at most $\tau$. Note that the family of subvarieties of $V$ of complexity at most $\tau$ forms a definable family; that is, there is $m \in \mathbb{N}$ and a constructible set $X \subset V \times K^{m}$ over $C$ such that every subvariety of $V$ over $K$ of complexity at most $\tau$ is of the form $X(\bar{b})=\{v:(v, \bar{b}) \in X\}$ for some $\bar{b} \in K^{m}$. In fact this is the only property we require of the notion of complexity.

2.1.11. Generic elements. Let $V$ as before be an algebraic variety over an algebraically closed $C \leq K$. For $a \in V(K)$ and $C^{\prime} \leq K$ an algebraically closed subfield containing $C$, we define the locus of $a$ over $C^{\prime}$ within $V$, $\operatorname{locus}^{V}\left(a / C^{\prime}\right)$, to be the smallest Zariski-closed subvariety of $V$ defined over $C^{\prime}$ and containing $a$. If $V(K) \subset K^{m}$ is affine and defined over $C$, then $\operatorname{locus}^{V}(a / C)=\operatorname{loc}^{0}(a / C)$.

If $V$ is irreducible, a point $a \in V(K)$ of $V$ is generic if it is contained in no proper closed subvariety over $C$, i.e. $\operatorname{locus}^{V}(a / C)=V$; equivalently, $\operatorname{trd}(a / C)=\operatorname{dim}(V)$.

Remark 2.11. If $V \subset \prod_{i} W_{i}$ and $V^{\prime} \subset \prod_{i} W_{i}^{\prime}$ are closed subvarieties where $V, V^{\prime}$, $W_{i}, W_{i}^{\prime}$ are irreducible varieties over $C_{0}$, then $V$ and $V^{\prime}$ are in co-ordinatewise correspondence if and only if they have generics $\bar{a} \in V(K)$ and $\bar{a}^{\prime} \in V^{\prime}(K)$ such that $a_{i} \in W_{i}(K)$ and $a_{i}^{\prime} \in W_{i}^{\prime}(K)$ are generic and for some permutation $\sigma \in \operatorname{Sym}(n)$, we have $\operatorname{acl}^{0}\left(a_{i}\right)=\operatorname{acl}^{0}\left(a_{\sigma i}^{\prime}\right)$. Indeed, $\operatorname{loc}^{0}\left(a_{i}, a_{\sigma i}^{\prime}\right) \subset W_{i} \times W_{\sigma i}^{\prime}$ is then a generically finite algebraic correspondence between $\operatorname{loc}^{0}\left(a_{i}\right)$ and $\operatorname{loc}^{0}\left(a_{\sigma i}\right)$, as required.

2.1.12. Canonical base. In the proof of our main theorems, it will be crucial to understand the dimensions of certain families of varieties. The right concept for this (which serves a similar purpose as the concept of Hilbert scheme in classical algebraic geometry) is the notion of canonical base.

Recall that the field of definition of a Zariski-closed subset $V \subset K^{n}$ is the smallest field $k$ over which $V$ is defined; equivalently (since $\operatorname{char}(K)=0), k$ is such that a field automorphism $\sigma \in \operatorname{Aut}(K)$ fixes $V$ setwise if and only if it fixes $k$ pointwise.

Given $\bar{a} \in K^{n}$ and $C \subset K^{<\infty}$ let $k \leq K$ be the field of definition of the absolutely irreducible Zariski-closed subset $\operatorname{loc}^{0}\left(\bar{a} / \operatorname{acl}^{0}(C)\right)$ of $K^{n}$. A tuple $\bar{d} \in K^{<\infty}$ is said to be a canonical base of $\bar{a}$ over $C$ if its co-ordinates together with $C_{0}$ generate the subfield of $K$ generated by $C_{0}$ and $k$.

Clearly if $\bar{d} \in K^{<\infty}$ is a canonical base of $\bar{a}$ over $C$ then it is a canonical base of $\bar{a}$ over $\operatorname{acl}^{0}(C)$ and conversely. Furthermore $\bar{d} \in \operatorname{acl}^{0}(C)$ and since $\operatorname{loc}^{0}\left(\bar{a} / \operatorname{acl}^{0}(C)\right)$ is defined over $C_{0}(\bar{d})$ we have:

$$
\operatorname{loc}^{0}\left(\bar{a} / \operatorname{acl}^{0}(C)\right)=\operatorname{loc}^{0}(\bar{a} / \bar{d})
$$

so $\operatorname{loc}^{0}(\bar{a} / \bar{d})$ is (absolutely) irreducible and

$$
\mathrm{d}^{0}(\bar{a} / \bar{d})=\mathrm{d}^{0}(\bar{a} / C)=\mathrm{d}^{0}\left(\bar{a} / \operatorname{acl}^{0}(C)\right)=\mathrm{d}^{0}(\bar{a} / C \bar{d}),
$$

in other words: $\bar{a} \downarrow \frac{0}{d} C$. In the proof of Proposition 5.14 below we shall require the following fact.

Lemma 2.12. Let $\bar{a} \in K^{<\infty}$ and $C \subset K^{<\infty}$. Let $\bar{d} \in K^{<\infty}$ be a canonical base of $\bar{a}$ over $C$ and $V:=\operatorname{loc}^{0}(\bar{a} \bar{d})$. Let $\bar{d}_{1}, \bar{d}_{2} \in \operatorname{tp}^{0}(\bar{d})(K)$ and $\bar{a}^{\prime} \in K^{<\infty}$ such that $\bar{a}^{\prime} \bar{d}_{i} \in V$. Then either $\mathrm{d}^{0}\left(\bar{a}^{\prime} / \bar{d}_{1} \bar{d}_{2}\right)<\mathrm{d}^{0}(\bar{a} / \bar{d})$, or $\bar{d}_{1}=\bar{d}_{2}$.

Proof. Note that

$$
\mathrm{d}^{0}\left(\bar{a}^{\prime} / \bar{d}_{1} \bar{d}_{2}\right) \leq \mathrm{d}^{0}\left(\bar{a}^{\prime} / \bar{d}_{1}\right)=\mathrm{d}^{0}\left(\bar{a}^{\prime} \bar{d}_{1}\right)-\mathrm{d}^{0}\left(\bar{d}_{1}\right) \leq \operatorname{dim} V-\mathrm{d}^{0}(\bar{d})=\mathrm{d}^{0}(\bar{a} / \bar{d}) .
$$


So if $\mathrm{d}^{0}\left(\bar{a}^{\prime} / \bar{d}_{1} \bar{d}_{2}\right) \geq \mathrm{d}^{0}(\bar{a} / \bar{d})$, then the above inequalities are equalities and in particular $\mathrm{d}^{0}\left(\bar{a}^{\prime} \bar{d}_{i}\right)=\mathrm{d}^{0}(\bar{a} \bar{d})$ for each $i$. Since $V$ is irreducible we obtain $V=\operatorname{loc}^{0}\left(\bar{a}^{\prime} \bar{d}_{i}\right)$. Hence there exist $\sigma_{i} \in \operatorname{Aut}\left(K / C_{0}\right)$ with $\sigma_{i}(\bar{a})=\bar{a}^{\prime}$ and $\sigma_{i}(\bar{d})=\bar{d}_{i}$.

Since $\bar{d}$ is a canonical base for $\bar{a}$ over $C, C_{0}(\bar{d})$ is the field of definition of $\operatorname{loc}^{0}(\bar{a} / \bar{d})$. Hence $C_{0}\left(\bar{d}_{i}\right)$ is the field of definition of $\operatorname{loc}^{0}\left(\bar{a}^{\prime} / \bar{d}_{i}\right)$, and thus $\bar{d}_{i}$ is a canonical base of $\bar{a}^{\prime}$ over $\bar{d}_{i}$. In particular $\operatorname{loc}^{0}\left(\bar{a}^{\prime} / \bar{d}_{i}\right)=\sigma_{i}\left(\operatorname{loc}^{0}(\bar{a} / \bar{d})\right)$ is irreducible. Since $\operatorname{loc}^{0}\left(\bar{a}^{\prime} / \bar{d}_{1} \bar{d}_{2}\right) \subset \operatorname{loc}^{0}\left(\bar{a}^{\prime} / \bar{d}_{i}\right)$ have the same dimension, we conclude that $\operatorname{loc}^{0}\left(\bar{a}^{\prime} / \bar{d}_{1} \bar{d}_{2}\right)=\operatorname{loc}^{0}\left(\bar{a}^{\prime} / \bar{d}_{i}\right)$.

In particular $\operatorname{loc}^{0}\left(\bar{a}^{\prime} / \bar{d}_{1}\right)=\operatorname{loc}^{0}\left(\bar{a}^{\prime} / \bar{d}_{2}\right)$. Setting $\sigma=\sigma_{2} \sigma_{1}^{-1}$, we get $\sigma\left(\bar{a}^{\prime}\right)=$ $\bar{a}^{\prime}, \sigma\left(\bar{d}_{1}\right)=\bar{d}_{2}$ and $\sigma\left(\operatorname{loc}^{0}\left(\bar{a}^{\prime} / \bar{d}_{1}\right)\right)=\operatorname{loc}^{0}\left(\bar{a}^{\prime} / \bar{d}_{2}\right)=\operatorname{loc}^{0}\left(\bar{a}^{\prime} / \bar{d}_{1}\right)$. Hence $\sigma$ fixes $\operatorname{loc}^{0}\left(\bar{a}^{\prime} / \bar{d}_{1}\right)$ setwise. It must fix its field of definition $C_{0}\left(\bar{d}_{1}\right)$ pointwise. Hence $\sigma\left(\bar{d}_{1}\right)=\bar{d}_{1}$, and $\bar{d}_{2}=\bar{d}_{1}$ as claimed.

2.1.13. Isogenies. We say that commutative algebraic groups $G_{1}, G_{2}$ are isogenous if there exists an isogeny $\theta: G_{1} \rightarrow G_{2}$; that is, a surjective algebraic group homomorphism with finite kernel. The relation of being isogenous is an equivalence relation.

We will apply in multiple places the following useful criterion for the existence of an isogeny.

Fact 2.13. Let $(G ; \times)$ and $\left(G^{\prime} ;+\right)$ be connected algebraic groups over an algebraically closed field $C_{0}$ of characteristic zero. Suppose the graphs $\Gamma_{\times}$and $\Gamma_{+}$of the group operations are in co-ordinatewise correspondence, and $G^{\prime}$ is commutative. Then $G$ is also commutative, and is isogenous to $G^{\prime}$.

Moreover, if $(g, h) \in G^{2}(K)$ and $\left(g^{\prime}, h^{\prime}\right) \in\left(G^{\prime}\right)^{2}(K)$ are each generic, and if $\operatorname{acl}^{0}(g)=\operatorname{acl}^{0}\left(g^{\prime}\right)$ and $\operatorname{acl}^{0}(h)=\operatorname{acl}^{0}\left(h^{\prime}\right)$ and $\operatorname{acl}^{0}(g \times h)=\operatorname{acl}^{0}\left(g^{\prime}+h^{\prime}\right)$ (where $\left.\operatorname{acl}^{0}(x)=C_{0}(x)^{\text {alg }}\right)$, then there are $n \in \mathbb{N}_{>0}$ and an isogeny $\alpha: G \rightarrow G^{\prime}$ and a point $c \in G^{\prime}\left(C_{0}\right)$ such that $\alpha g=n g^{\prime}+c$.

Proof. This is a consequence of [BMP14, Lemme 2.4]. Indeed, that lemma yields, via Remark 2.11, that there is an algebraic subgroup $S \leq G \times G^{\prime}$ such that the projections to $G$ and $G^{\prime}$ are surjective and have finite kernels. It follows that $G$ is abelian. Indeed, if $g \in G$ then $g^{G}$ is finite, so the centraliser $C_{g}$ is a finite index subgroup of $G$ and hence is equal to $G$ since the latter is connected. Alternatively, one may assume by the Lefschetz principle that $G, G^{\prime}$, and $S$ are complex algebraic groups, and observe that $S$ induces an isomorphism of the Lie algebras.

Now let $n$ be the exponent of the subgroup $\operatorname{coker}(S):=\left\{y \in G^{\prime}:(0, y) \in S\right\} \leq$ $G^{\prime}$. Then by setting $\alpha(x):=n y$ whenever $(x, y) \in S$, we obtain a well-defined isogeny $\alpha: G \rightarrow G^{\prime}$. So $G$ is isogenous to $G^{\prime}$.

For the "moreover" clause, we use that the subgroup $S$ in the above cited lemma is a coset of $V:=\operatorname{locus}^{G \times G^{\prime}}\left(\left(g, g^{\prime}\right) / C_{0}\right)$. Knowing that $G$ is abelian, we can see this fairly directly as follows. Let $S$ be the stabiliser of $\operatorname{tp}^{0}\left(g, g^{\prime}\right)$, namely $S:=\{\gamma \in$ $\left.G \times G^{\prime}: \operatorname{dim}(V \cap(\gamma+V))=\operatorname{dim}(V)\right\}$. Then $S$ projects surjectively with finite kernel to $G$ and to $G^{\prime}$, and it follows from our assumptions that $V$ is a coset of $S$; indeed, this can be seen by applying [Zie06, Theorem 1] to $\left(g, g^{\prime}\right)+\left(h, h^{\prime}\right)=\left(g \times h, g^{\prime}+h^{\prime}\right)$.

Since $C_{0}$ is algebraically closed and both $V$ and $S$ are over $C_{0}$, there is $\left(c_{1}, c_{2}\right) \in$ $\left(G \times G^{\prime}\right)\left(C_{0}\right)$ such that $V=\left(c_{1}, c_{2}\right)+S$. Then since the projection $\pi_{1}: S \rightarrow G$ is surjective, there exists $c^{\prime} \in G^{\prime}\left(C_{0}\right)$ such that $\left(g, g^{\prime}+c^{\prime}\right) \in S$, namely any $c^{\prime}$ such that $\left(c_{1}, c_{2}-c^{\prime}\right) \in S$. Then with $\alpha, n$ as above, we have $\alpha(g)=n\left(g^{\prime}+c^{\prime}\right)=n g^{\prime}+n c^{\prime}$, so $c:=n c^{\prime}$ is as required.

2.2. Incidence bounds and Szemerédi-Trotter. As in [ES12] we will require some incidence bounds à la Szemerédi-Trotter in higher dimension. As is wellknown, if $\mathcal{G}$ is a bi-partite graph between vertex sets $X_{1}$ and $X_{2}$ with the property 
that no two distinct points in $X_{2}$ have more than $B$ common neighbours, then a simple argument via the Cauchy-Schwarz inequality (e.g. see [ES12, Prop. 12]), implies that the number of edges of $\mathcal{G}$ is at most $O\left(\left|X_{1}\right|^{\frac{1}{2}}\left|X_{2}\right|+B\left|X_{1}\right|\right)$. The theorem of Szemerédi-Trotter and its generalisations (such as [PS98], [ES12, Theorem 9] or more recently $\left[\mathrm{FPS}^{+} 17\right.$, Theorem 1.2]) aim at improving this inequality by some power saving in the situation when the vertex sets $X_{1}$ and $X_{2}$ are points in Euclidean space and the graph $\mathcal{G}$ is given by some algebraic relation. For example Elekes-Szabó prove the following Szemerédi-Trotter-type result:

Theorem 2.14 ([ES12, Theorem 9]). If $V \subset \mathbb{C}^{n_{1}} \times \mathbb{C}^{n_{2}}$ is a complex algebraic subvariety there is $\epsilon_{0}=\epsilon_{0}\left(n_{2}\right)>0$ such that the following holds. Let $B \in \mathbb{N}$. Let $X_{1}, X_{2} \subset \mathbb{C}^{n}$ be finite subsets. Write $V(y):=\left\{x \in \mathbb{C}^{n}:(x, y) \in V\right\}$ for the fiber above $y \in \mathbb{C}^{n}$. Assume that for any two distinct $y, y^{\prime} \in X_{2}$ the intersection $V(y) \cap V\left(y^{\prime}\right)$ contains at most $B$ points from $X_{1}$. Then the number $I$ of incidences $(x, y) \in X_{1} \times X_{2}$ with $x \in V(y)$ satisfies:

$$
I \leq O_{B, V, n_{1}}\left(\left|X_{1}\right|^{\frac{1}{2}\left(1+\epsilon_{0}\right)}\left|X_{2}\right|^{1-\epsilon_{0}}+\left|X_{1}\right|+\left|X_{2}\right| \log \left|X_{1}\right|\right) .
$$

We note that this bound has been slightly improved, with a better $\epsilon_{0}$ (namely any $\epsilon_{0}<\frac{1}{4 n_{2}-1}$ ) and no log factor in $\left[\mathrm{FPS}^{+} 17\right.$, Theorem 1.2].

Looking carefully at the proofs of the above theorem we find that the dependence in $B$ of the big- $O$ is sublinear, that is $O_{B, V, n_{1}} \leq B \cdot O_{V, n_{1}}$ (see [She19, Problem 11.4]). This aspect will be important for us (we can afford a polynomial dependence).

In what follows we spell out how the above incidence bound reads in the formalism of coarse pseudo-finite dimension. With the notation and terminology of Section 2.1 (in particular $K$ is an ultraproduct of fields of characteristic zero and $\boldsymbol{\delta}$ is the coarse dimension 2.1.3), we have:

Lemma 2.15 (Szemerédi-Trotter-type bound). Let $X_{1} \subset K^{n_{1}}$ and $X_{2} \subset K^{n_{2}}$, suppose each $X_{i}$ is $\bigwedge$-internal, and let $X=\left(X_{1} \times X_{2}\right) \cap V$ where $V \subset K^{n_{1}+n_{2}}$ is a K-Zariski closed subset. Assume that $\boldsymbol{\delta}\left(X_{1}\right), \boldsymbol{\delta}\left(X_{2}\right)$ are both finite. Set

$$
\beta:=\sup _{a, b \in X_{2} ; a \neq b} \delta(X(a) \cap X(b)),
$$

where $X(y):=\left\{x \in X_{1}:(x, y) \in X\right\}$. Then for some $\epsilon_{0}>0$ depending only on $n_{2}$, writing $y^{+}:=\max \{0, y\}$,

$$
\boldsymbol{\delta}(X) \leq \beta+\max \left(\frac{1}{2} \boldsymbol{\delta}\left(X_{1}\right)+\boldsymbol{\delta}\left(X_{2}\right)-\epsilon_{0}\left(\boldsymbol{\delta}\left(X_{2}\right)-\frac{1}{2} \boldsymbol{\delta}\left(X_{1}\right)\right)^{+}, \boldsymbol{\delta}\left(X_{1}\right), \boldsymbol{\delta}\left(X_{2}\right)\right) .
$$

Remark 2.16. In the same way, the trivial bound mentioned earlier (via CauchySchwarz) yields the same estimate on $\boldsymbol{\delta}(X)$ as above, but with $\epsilon_{0}=0$. The original Szemerédi-Trotter theorem [ST83] corresponds to the case when $X_{1}$ is the ultraproduct of finite sets of points in $\mathbb{R}^{2}$ and $X_{2}$ is the ultraproduct of finite sets of lines in $\mathbb{R}^{2}$, and $V$ is the incidence relation $p \in \ell$. In this case $\epsilon_{0}=\frac{1}{3}$, which is optimal.

Proof of Lemma 2.15. Suppose first that $X_{1}$ and $X_{2}$ are internal sets, i.e. $X_{i}=$ $\prod_{s \rightarrow \mathcal{U}} X_{i}^{K_{s}}$ for $i=1,2$, for some $X_{i}^{K_{s}} \subset K_{s}^{n_{i}}$, and $X^{K_{s}}=\left(X_{1}^{K_{s}} \times X_{2}^{K_{s}}\right) \cap V\left(K_{s}\right)$. Since $\boldsymbol{\delta}\left(X_{i}\right)$ is finite, $X_{i}^{K_{s}}$ is finite for $\mathcal{U}$-almost every $s$. The assumption $\boldsymbol{\delta}(X(a) \cap$ $X(b)) \leq \beta$ for each $a, b \in X_{2}$ implies that for each $\epsilon>0$ for $\mathcal{U}$-almost every $s$ we have:

$$
\left|X^{K_{s}}(a) \cap X^{K_{s}}(b)\right| \leq B_{s},
$$

where $B_{s}:=\xi_{s}^{\beta+\epsilon}$, and $\xi=\lim _{s \rightarrow \mathcal{U}} \xi_{s}$ is the scaling constant as in $\S 2.1 .3$. Now Theorem 2.14 implies:

$$
\left|X^{K_{s}}\right| \leq B_{s} \cdot O_{V, n_{1}}\left(\left|X_{1}^{K_{s}}\right|^{\frac{1}{2}\left(1+\epsilon_{0}\right)}\left|X_{2}^{K_{s}}\right|^{1-\epsilon_{0}}+\left|X_{1}^{K_{s}}\right|+\left|X_{2}^{K_{s}}\right| \log \left|X_{1}^{K_{s}}\right|\right) .
$$


Taking logarithms and passing to the ultralimit yields the desired bound.

Finally the following claim allows us to reduce to the case when $X_{i}$ are internal sets:

Claim 2.17. For any $\beta^{\prime}>\beta$, there are internal subsets $X_{i}^{\prime} \supset X_{i}$, for $i=1,2$, such that for all $a, b \in X_{2}^{\prime}$ with $a \neq b$, we have $\delta\left(X^{\prime}(a) \cap X^{\prime}(b)\right)<\beta^{\prime}$, where $X^{\prime}:=\left(X_{1}^{\prime} \times X_{2}^{\prime}\right) \cap V$.

Proof. The variety $V$ is defined over a countable (finitely generated) subfield of $K$, which we denote by $k$. Since each $X_{i}$ is $\bigwedge$-internal, we may work in a language $\mathcal{L}$ in which each $X_{i}$ is $\bigwedge$-definable and $\boldsymbol{\delta}$ is continuous. Note that $X(y)=X_{1} \cap V(y)$ for each $y \in X_{2}$. Since $X_{1}$ is $\Lambda$-definable, in view of (2), for any $a, b \in X_{2}$ with $a \neq b$ there is a $\emptyset$-definable subset $X_{1}^{a, b} \supset X_{1}$ such that $\boldsymbol{\delta}\left(X_{1}^{a, b} \cap V(a) \cap V(b)\right)<\beta^{\prime}$. By continuity of $\boldsymbol{\delta}$ (see 2.1.6 and Remark 2.7), there is a $k$-definable subset of $Z^{a, b}$ of $K^{n_{2}} \times K^{n_{2}}$ containing $(a, b)$ such that $\delta\left(X_{1}^{a, b} \cap V\left(a^{\prime}\right) \cap V\left(b^{\prime}\right)\right)<\beta^{\prime}$ for all $\left(a^{\prime}, b^{\prime}\right) \in Z^{a, b}$. Hence $\left(X_{2}\right)^{2} \backslash \Delta$ (where $\Delta$ denotes the diagonal) is covered by the family of $k$-definable sets $Z^{a, b}$. This is a countable family, because there are only countably many $k$-definable sets. Combined with the fact that $X_{2}$ is $\Lambda$ definable, $\aleph_{1}$-compactness (see 2.1.2) now implies that there must be a $\emptyset$-definable set $X_{2}^{\prime}$ containing $X_{2}$ such that $\left(X_{2}^{\prime}\right)^{2} \backslash \Delta$ is contained in finitely many $Z^{a, b}$ 's, say $Z^{a_{1}, b_{1}}, \ldots, Z^{a_{m}, b_{m}}$. Let $X_{1}^{\prime}$ be the intersection of the corresponding $X_{1}^{a_{i}, b_{i}}$, $i=1, \ldots, m$. Then by monotonicity of coarse dimension $\boldsymbol{\delta}\left(X_{1}^{\prime} \cap V\left(a^{\prime}\right) \cap V\left(b^{\prime}\right)\right)<\beta^{\prime}$ for all $a^{\prime}, b^{\prime} \in\left(X_{2}^{\prime}\right)^{2} \backslash \Delta$. So $X_{1}^{\prime}$ and $X_{2}^{\prime}$ are as desired.

\section{Warm-UP: the Elekes-Szabó theorem}

In this section, we show how the proof of the original Elekes-Szabó theorem translates in the non-standard setup expounded in the previous section. This will help us motivate the notions introduced in the following section, where we will pass to the general case of arbitrary dimension and arity and work towards Theorems 1.4 and 1.11. We begin with the one-dimensional case, i.e. we prove Theorem 1.2. A similar result was proven by Hrushovski using similar techniques as [Hru13, Proposition 5.21]. We then proceed to recover Elekes-Szabó's second theorem, which corresponds to the case of a $2 d$-dimensional variety in $\left(\mathbb{C}^{d}\right)^{3}$, and at the same time add two things: we establish that the associated algebraic group is in fact commutative (this was noted already in [BW16]), and we also give an explicit gap in the power-saving, $\frac{1}{16}$ in fact. Although this is indeed new, we include this section mostly for the reader's convenience as a way to introduce some of the ideas in a special case. But a reader only interested in the proof of Theorems 1.4 and 1.11 may safely skip ahead to Section 5 .

\subsubsection{Abelian group configuration theorem.}

While Elekes-Szabó used their 'composition lemma' to establish the existence of the associated algebraic group, we will rely directly on the Group Configuration Theorem. This is a by now classical theorem of model theory due to Zilber and Hrushovski. We first recall its statement in the form we need and then describe a variant, due to Hrushovski, which ensures that the associated group is commutative. In this paragraph $C_{0} \leq K$ are arbitrary algebraically closed fields, and we use the notation of $\S 2.1 .7$, in particular $K^{<\infty}=\cup_{n>0} K^{n}$ and $\operatorname{acl}^{0}(A)$ is the algebraic closure of $C_{0}(A)$ in $K$.

Theorem 3.1 (Group Configuration Theorem). Suppose $a, b, c, x, y, z \in K^{<\infty}$ are such that in the following diagram 




for any three distinct points $a_{1}, a_{2}, a_{3}$,

- if $a_{1}, a_{2}, a_{3}$ lie on a common line then $a_{i} \in \operatorname{acl}^{0}\left(a_{j}, a_{k}\right)$ whenever $\{i, j, k\}=$ $\{1,2,3\}$,

- if $a_{1}, a_{2}, a_{3}$ do not lie on a common line then $a_{i} \mathfrak{L}^{0} a_{j} a_{k}$ whenever $\{i, j, k\}=$ $\{1,2,3\}$.

Then there is a connected algebraic group $(G, \cdot)$ defined over $C_{0}$, and generic elements $a^{\prime}, b^{\prime}, c^{\prime} \in G(K)$ such that each primed element is $\operatorname{acl}^{0}$-interalgebraic with the corresponding unprimed element, namely $\operatorname{acl}^{0}(x)=\operatorname{acl}^{0}\left(x^{\prime}\right)$ for each $x \in\{a, b, c\}$, and $c^{\prime}=b^{\prime} \cdot a^{\prime}$.

Remark 3.2. Here, $\operatorname{acl}^{0}\left(x^{\prime}\right)$ is to be understood via a coding of elements of $G(K)$ as tuples from $K$, as discussed in 2.1.10. But since $x^{\prime}$ is generic, we may equivalently fix a single arbitrary affine patch over $C_{0}$ and take co-ordinates there.

For a proof of this theorem, we refer the reader to [Pil96, Theorem 5.4.5, Remark 5.4.10]. Strictly speaking only a $\bigwedge$-definable (in ACF) group $G^{\prime}$ satisfying desired conclusions is obtained there, but by [Pi196, Remark 1.6.21], $G^{\prime}$ is in fact definable (in ACF). By the Van den Dries-Hrushovski-Weil theorem [Pi198, Theorem 4.12] any such group is definably isomorphic over $C_{0}$ to an algebraic group $G$ as required.

Theorem 3.3 (Abelian Group Configuration Theorem). Suppose $a, b, c, w, x, y, z \in$ $K^{<\infty}$ are such that in the following diagram

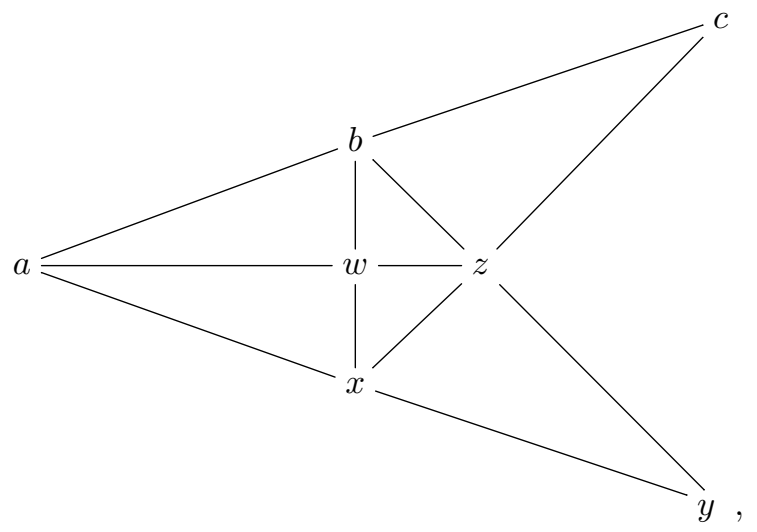

for any three distinct points $a_{1}, a_{2}, a_{3}$,

- if $a_{1}, a_{2}, a_{3}$ lie on a common line then $a_{i} \in \operatorname{acl}^{0}\left(a_{j}, a_{k}\right)$ whenever $\{i, j, k\}=$ $\{1,2,3\}$, 
- if $a_{1}, a_{2}, a_{3}$ do not lie on a common line and $\left\{a_{1}, a_{2}, a_{3}\right\} \neq\{w, c, y\}$ then $a_{i} \downarrow^{0} a_{j} a_{k}$ whenever $\{i, j, k\}=\{1,2,3\}$.

Then there is an connected commutative algebraic group $G$ defined over $C_{0}$, and generics $a^{\prime}, b^{\prime}, c^{\prime} \in G(K)$ such that each primed element is $\operatorname{acl}^{0}$-interalgebraic with the corresponding unprimed element, and $c^{\prime}=b^{\prime}+a^{\prime}$.

Note that the hypotheses of Theorem 3.1 are satisfied, so we need only show that our additional assumptions yield that the algebraic group $G$ obtained from that theorem is commutative. We refer to [BHM17, Theorem C.1] for a proof of this.

3.0.2. Elekes-Szabó - one dimensional case. In this paragraph we reprove the original Elekes-Szabó theorem, namely Theorem 1.2. We start by reformulating it in the non-standard setup of the last section; in particular we keep the notation of Section 2.1. So $K$ is an ultrapower of the complex field, $\boldsymbol{\delta}$ is the coarse dimension 2.1.3 which is continuous in a countable language $\mathcal{L}$ containing $\mathcal{L}_{\text {ring }}$ and constant symbols for each element of the countable algebraically closed field $C_{0}$ over which $V$ is defined, and $\mathrm{d}^{0}$ denotes transcendence degree over $C_{0}$.

Theorem 3.4 (Reformulation of Theorem 1.2). Let $a_{1}, a_{2}, a_{3} \in K$ and assume that for all $i \neq j, \operatorname{dim}^{0}\left(a_{i}, a_{j}\right)=\operatorname{dim}^{0}\left(a_{1}, a_{2}, a_{3}\right)=2, \boldsymbol{\delta}\left(a_{i}\right) \leq 1$ and $\boldsymbol{\delta}\left(a_{1}, a_{2}, a_{3}\right)=$ 2 . Then there exists a connected one-dimensional algebraic group $G$ over $C_{0}$ and $a_{1}^{\prime}, a_{2}^{\prime}, a_{3}^{\prime} \in G(K)$ with $\operatorname{acl}^{0}\left(a_{i}\right)=\operatorname{acl}^{0}\left(a_{i}^{\prime}\right)$ for $i=1,2,3$ and $a_{3}^{\prime}=a_{1}^{\prime}+a_{2}^{\prime}$.

Proof of Theorem 1.2 from Theorem 3.4. Assume $V \subset \mathbb{C}^{3}$ does not project to a curve on two co-ordinates and has no power-saving. Then we may find a sequence of positive integers $\left(N_{s}\right)_{s \geq 0}$ with $\lim _{s \rightarrow \infty} N_{s}=+\infty$ and finite subsets $X_{1}^{s}, X_{2}^{s}$ and $X_{3}^{s}$ in $\mathbb{C}$ with $\left|X_{i}^{s}\right| \leq N_{s}$ for each $i, s$ such that

$$
\left|X_{1}^{s} \times X_{2}^{s} \times X_{3}^{s} \cap V\right| \geq N_{s}^{2-\epsilon_{s}}
$$

for some $\epsilon_{s}>0$ with $\lim _{s \rightarrow \infty} \epsilon_{s}=0$. Passing to an ultraproduct $X_{i}=\prod_{s \rightarrow \mathcal{U}} X_{i}^{s}$ for some non-principal ultrafilter $\mathcal{U}$ over the integers, we obtain three internal sets $X_{i} \subset K$, where $K$ is the ultrapower of $\mathbb{C}$, and we define the coarse dimension $\boldsymbol{\delta}$ as in 2.1 .3 with scaling constant $\xi=\lim _{s \rightarrow \mathcal{U}} N_{s}$. Hence $\boldsymbol{\delta}\left(X_{i}\right) \leq 1$ for each $i$ and $\boldsymbol{\delta}\left(X_{1} \times X_{2} \times X_{3} \cap V\right)=2$.

Since $V$ is irreducible and does not project to a curve on two co-ordinates, the fibers of co-ordinate projections of $V$ on pairs of co-ordinates have uniformly bounded size. Consequently $\left|X_{1}^{s} \times X_{2}^{s} \times X_{3}^{s} \cap V\right|=O\left(\left|X_{i}^{s} \times X_{j}^{s}\right|\right)$ for all $s$ and all $i \neq j$. It follows that $2=\boldsymbol{\delta}\left(X_{1} \times X_{2} \times X_{3} \cap V\right) \leq \boldsymbol{\delta}\left(X_{i}\right)+\boldsymbol{\delta}\left(X_{j}\right)$, and hence that $\boldsymbol{\delta}\left(X_{i}\right)=1$ for each $i$.

The variety $V$ is defined over some finitely generated subfield of $\mathbb{C}$. Let $C_{0}$ be its algebraic closure in $\mathbb{C}$. It is a countable subfield. To be able to talk about definable sets we specify a language $\mathcal{L}$ as follows: we start with $\mathcal{L}_{\text {ring }}=(K,+, \cdot, 0,1)$ the language of rings and enlarge it by adding a constant symbol for each element of $C_{0}$ as well as a predicate for each $X_{i}, i=1,2,3$, thus in effect forcing $X_{i}$ to be definable. Finally we enlarge $\mathcal{L}$ as in $\S 2.1 .6$ so as to make $\boldsymbol{\delta}$ continuous and hence additive.

Now by Fact 2.4 we may find a triple $\left(a_{1}, a_{2}, a_{3}\right) \in X_{1} \times X_{2} \times X_{3} \cap V$ such that $\boldsymbol{\delta}\left(a_{1}, a_{2}, a_{3}\right)=\boldsymbol{\delta}\left(X_{1} \times X_{2} \times X_{3} \cap V\right)=2$. Note that $\left(a_{1}, a_{2}, a_{3}\right)$ is generic in $V$, i.e. it is not contained in any proper algebraic subvariety over the base field $C_{0}$, because $\left|X_{1}^{s} \times X_{2}^{s} \times X_{3}^{s} \cap W\right|=O_{W}\left(N_{s}\right)$ for every one-dimensional subvariety $W \subsetneq V$ over $C_{0}$, and so $\delta\left(X_{1} \times X_{2} \times X_{3} \cap W\right) \leq 1$. Consequently $\mathrm{d}^{0}\left(a_{1}, a_{2}, a_{3}\right)=\mathrm{d}^{0}\left(a_{i}, a_{j}\right)=2$ for all $i \neq j$. So we are in the situation of Theorem 3.4. Then $\operatorname{loc}^{0}\left(a_{1}^{\prime}, a_{2}^{\prime}, a_{3}^{\prime}\right)$ is the graph $\Gamma_{G}(\mathbb{C})$ of the group operation of $G$, and we conclude that $V$ has the required description via the correspondence $\operatorname{loc}^{0}\left(\left(a_{1}, a_{2}, a_{3}\right),\left(a_{1}^{\prime}, a_{2}^{\prime}, a_{3}^{\prime}\right)\right) \subset V \times \Gamma_{G}(\mathbb{C})$, which 
is defined over $C_{0}$ and projects to the correspondences given by the (irreducible) curves $\operatorname{loc}^{0}\left(a_{i}, a_{i}^{\prime}\right) \subset \mathbb{C} \times G(\mathbb{C})$.

We now pass to the proof of Theorem 3.4. We need to verify that the hypotheses of the group configuration are met. For this we crucially need the following lemma, which can be interpreted as saying that a 2-parameter family of plane curves with no power-saving must in fact be one-dimensional. This is where the Szemerédi-Trotter bound comes into play.

Lemma 3.5. Let $x_{1}, \ldots, x_{4} \in K$ be such that $\boldsymbol{\delta}\left(x_{i}\right)=1$ and $\boldsymbol{\delta}\left(x_{1}, \ldots, x_{4}\right)=$ $\mathrm{d}^{0}\left(x_{1}, \ldots, x_{4}\right)$. Assume that $\mathrm{d}^{0}\left(x_{1}, x_{2} / x_{3}, x_{4}\right)=1$. Then there is $x_{5} \in \operatorname{acl}^{0}\left(x_{3}, x_{4}\right)$ with $\boldsymbol{\delta}\left(x_{5}\right)=\mathrm{d}^{0}\left(x_{5}\right)=1$ such that $\mathrm{d}^{0}\left(x_{1}, x_{2} / x_{5}\right)=1$.

Proof. We postpone the proof of this lemma to the next subsection, where a stronger quantitative version of it will be proven as Lemma 3.11. It is also a special case of Proposition 5.14.

Proof of Theorem 3.4. First note that the assumptions imply that $\boldsymbol{\delta}\left(a_{i}\right)=1$ for each $i$. Indeed for any three distinct $i, j, k$ we have $a_{i} \in \operatorname{acl}^{0}\left(a_{j}, a_{k}\right)$. Hence by (7) we have $\boldsymbol{\delta}\left(a_{i} / a_{j}, a_{k}\right)=0$. And by additivity of $\boldsymbol{\delta}$ (see Fact 2.8) we get

$$
\boldsymbol{\delta}\left(a_{i}, a_{j}, a_{k}\right)=\boldsymbol{\delta}\left(a_{i} / a_{j}, a_{k}\right)+\boldsymbol{\delta}\left(a_{j}, a_{k}\right)=\boldsymbol{\delta}\left(a_{j}, a_{k}\right) \leq \boldsymbol{\delta}\left(a_{j}\right)+\boldsymbol{\delta}\left(a_{k}\right) .
$$

This forces $\boldsymbol{\delta}\left(a_{j}\right)$ and $\boldsymbol{\delta}\left(a_{k}\right)$ to be equal to 1 , since both are $\leq 1$.

Let $X=\operatorname{tp}\left(a_{2}, a_{3} / a_{1}\right)(K)$ be the set of realisations of the type of the pair $\left(a_{2}, a_{3}\right)$ over $a_{1}$, namely the intersection of all definable sets over $C:=\left\{a_{1}\right\}$ containing $\left(a_{2}, a_{3}\right)$. By additivity of $\boldsymbol{\delta}$ we have $\boldsymbol{\delta}(X)=\boldsymbol{\delta}\left(a_{1}, a_{2}, a_{3}\right)-\boldsymbol{\delta}\left(a_{1}\right)=2-1=1$, by assumption. According to Fact 2.4 we can find $\left(a_{4}, a_{5}\right) \in X$ such that

$$
\boldsymbol{\delta}\left(a_{4}, a_{5} / a_{1}, a_{2}, a_{3}\right)=\boldsymbol{\delta}(X)=1 .
$$

We will show that there is $a_{6} \in K$ such that $a_{1}, \ldots, a_{6}$ satisfy the hypotheses of the group configuration theorem as in the following diagram:

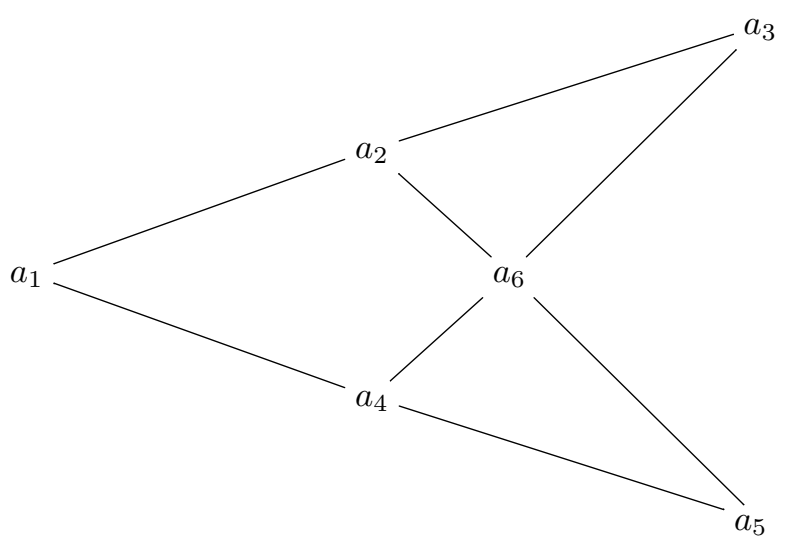

Since $\left(a_{4}, a_{5}\right)$ and $\left(a_{2}, a_{3}\right)$ have the same type over $a_{1}$, they have the same type over the empty set, and in particular they belong to the same algebraic subsets of $K^{2}$ defined over $C_{0}$. So d $\mathrm{d}^{0}\left(a_{4}, a_{5}, a_{1}\right)=2, \mathrm{~d}^{0}\left(a_{4}, a_{5}\right)=\mathrm{d}^{0}\left(a_{4}, a_{1}\right)=\mathrm{d}^{0}\left(a_{5}, a_{1}\right)=2$.

Moreover the Zariski dimension of the whole system is 3 , i.e. $\mathrm{d}^{0}\left(a_{1}, \ldots, a_{5}\right)=3$. Indeed it is at most 3 given that $a_{5} \in \operatorname{acl}^{0}\left(a_{1}, a_{4}\right)$ and $a_{3} \in \operatorname{acl}^{0}\left(a_{1}, a_{2}\right)$, but it cannot be less, for otherwise $\mathrm{d}^{0}\left(a_{4}, a_{5} / a_{1}, a_{2}, a_{3}\right)=0$ forcing $\boldsymbol{\delta}\left(a_{4}, a_{5} / a_{1}, a_{2}, a_{3}\right)=0$ by $(7)$, a contradiction to $(10)$.

$$
\text { Claim: } \quad \mathrm{d}^{0}\left(a_{3}, a_{4}\right)=2, \mathrm{~d}^{0}\left(a_{2}, a_{5}\right)=2 \text { and } \mathrm{d}^{0}\left(a_{2}, a_{5} / a_{3}, a_{4}\right)=1 .
$$


Indeed if $\mathrm{d}^{0}\left(a_{3}, a_{4}\right)<2$, then $a_{4} \in \operatorname{acl}^{0}\left(a_{3}\right)$, and thus

$$
\mathrm{d}^{0}\left(a_{1}, \ldots, a_{5}\right)=\mathrm{d}^{0}\left(a_{1}, a_{2}, a_{3}, a_{4}\right)=\mathrm{d}^{0}\left(a_{1}, a_{2}, a_{3}\right)=2,
$$

where we have used that $a_{5} \in \operatorname{acl}^{0}\left(a_{1}, a_{4}\right)$. In a similar way $\mathrm{d}^{0}\left(a_{2}, a_{5}\right)=2$. Now by additivity of $\mathrm{d}^{0}$ we finally get $\mathrm{d}^{0}\left(a_{2}, a_{5} / a_{3}, a_{4}\right)=1$, proving the claim.

Further note that by additivity and (10) we have

$$
\boldsymbol{\delta}\left(a_{2}, a_{3}, a_{4}, a_{5}\right)=\boldsymbol{\delta}\left(a_{4}, a_{5} / a_{2}, a_{3}\right)+\boldsymbol{\delta}\left(a_{2}, a_{3}\right)=1+2=3=\mathrm{d}^{0}\left(a_{2}, a_{3}, a_{4}, a_{5}\right) .
$$

So Lemma 3.5 applies and gives $a_{6} \in \operatorname{acl}^{0}\left(a_{3}, a_{4}\right)$ such that $\mathrm{d}^{0}\left(a_{2}, a_{5} / a_{6}\right)=1$ and $\mathrm{d}^{0}\left(a_{6}\right)=1$. It then follows easily by additivity of $\mathrm{d}^{0}$ that $\mathrm{d}^{0}\left(a_{6}, a_{2}\right)=\mathrm{d}^{0}\left(a_{5}, a_{6}\right)=2$ and $a_{6} \in \operatorname{acl}^{0}\left(a_{2}, a_{5}\right)$. This shows that $a_{1}, \ldots, a_{6}$ satisfy the hypotheses of the group configuration theorem. We are done.

3.0.3. Coarse general position. A significant new difficulty arises when dealing with the higher dimensional situation, i.e. when $m=\operatorname{dim} W_{i}>1$ say in Theorem 1.11. We will have to assume that the finite sets $X_{i} \subset W_{i}$ do not have too large an intersection with proper subvarieties. There are various ways to quantify this assumption, for instance Elekes-Szabó's notion of general position requires that the intersections have bounded size with a bound depending only on the complexity of the subvariety. We will adopt here the weaker assumption of coarse general position. As explained in Section 4 below, some assumption of this kind is necessary for the result to hold.

Recall from Definition 1.7 that for $\tau \in \mathbb{N}$, a finite subset $X$ of a complex algebraic variety $W$ is said to be in coarse $(C, \tau)$-general position (or $(C, \tau)$-cgp for short) with respect to $W$ if $\left|W^{\prime} \cap X\right| \leq|X|^{\frac{1}{\tau}}$ for any proper irreducible complex subvariety $W^{\prime} \subsetneq W$ of complexity at most $C \in \mathbb{N}$. In the non-standard setup of Section 2 , where we have specified a language $\mathcal{L}$ and defined the coarse dimension $\boldsymbol{\delta}$, it will be convenient to define a notion of coarse general position for tuples $\bar{a} \in K^{<\infty}$. We will say that $\bar{a} \in K^{<\infty}$ is in coarse general position or is $\operatorname{cg} \boldsymbol{p}$ for short if for every $\bar{b} \in K^{<\infty}$ such that $\bar{a}$ is not independent from $\bar{b}$, that is such that $\mathrm{d}^{0}(\bar{a} / \bar{b})<\mathrm{d}^{0}(\bar{a})$, we have:

$$
\boldsymbol{\delta}(\bar{a} / \bar{b})=0 .
$$

The two notions are closely related as follows. Suppose $W \subset \mathbb{C}^{n}$ is a variety and $X=\prod_{s \rightarrow \mathcal{U}} X_{s} \subset W(K)$ is an internal set. Assume that $X$ is definable without parameters in the countable language $\mathcal{L}$ of Section 2 for which the coarse dimension $\boldsymbol{\delta}$ is continuous (see 2.1.6).

Lemma 3.6. Suppose that $0<\boldsymbol{\delta}(X)<\infty$ and that for any $\tau \in \mathbb{N}$, there is $C \geq \tau$ such that for $\mathcal{U}$-many $s, X_{s}$ is $(C, \tau)$-cgp in $W(\mathbb{C})$. Then any tuple $\bar{a} \in K^{n}$ lying in $X$ is cgp.

Proof. Let $\bar{b} \in K^{<\infty}$ such that $\mathrm{d}^{0}(\bar{a} / \bar{b})<\mathrm{d}^{0}(\bar{a})$. Then setting $W^{\prime}=\operatorname{loc}^{0}\left(\bar{a} / \operatorname{acl}^{0}(\bar{b})\right)$ we get an absolutely irreducible subvariety of $W$, which is proper, since by (8) one has $\operatorname{dim}\left(W^{\prime}\right)=\mathrm{d}^{0}(\bar{a} / \bar{b})<\operatorname{dim} W$, and contains $\bar{a}$. Let $c$ be the complexity of $W^{\prime}$. Then for every $\tau>c, \mathcal{U}$-many $X_{s}$ are $(c, \tau)$-cgp in $W(\mathbb{C})$, and this implies that $\boldsymbol{\delta}\left(\bar{a} / \operatorname{acl}^{0}(\bar{b})\right) \leq \boldsymbol{\delta}\left(X \cap W^{\prime}(K)\right)=0$. Hence $\boldsymbol{\delta}(\bar{a} / \bar{b})=0$ by $(7)$.

Remark 3.7. The property of being cgp for a tuple $\bar{a} \in K^{m}$ depends only on the type $\operatorname{tp}(\bar{a})$ of $\bar{a}$. Indeed, suppose $\bar{a}^{\prime} \in K^{m}$ has the same type, and $\bar{b} \in K^{n}$ is such that $\bar{a}^{\prime} £^{0} \bar{b}$. Then there is $\bar{b}^{\prime} \in K^{n}$ such that $\operatorname{tp}\left(\bar{a}, \bar{b}^{\prime}\right)=\operatorname{tp}\left(\bar{a}^{\prime}, \bar{b}\right)$, by $\aleph_{1}$-compactness of $K$. So $\bar{a} \swarrow^{0} \bar{b}^{\prime}$, and so $\boldsymbol{\delta}\left(\bar{a} / \bar{b}^{\prime}\right)=0$. But then by invariance of $\boldsymbol{\delta}$ (see 2.8ii.), we have $\boldsymbol{\delta}\left(\bar{a}^{\prime} / \bar{b}\right)=\boldsymbol{\delta}(\bar{a} / \bar{b})=0$. 
3.0.4. Higher dimensional case: Elekes-Szabó with a gap and commutativity. We now move on to the second theorem proved by Elekes-Szabó in [ES12], which is the extension of Theorem 1.2 to higher dimensional varieties. We give a proof following the strategy used above in the one-dimensional case. As a payoff we will also get an explicit bound, $\frac{1}{16}$, on the power-saving and we will establish that the group involved must be commutative. This feature (rather the nilpotency) had been hinted at already by Elekes-Szabó (see their Example 28 in [ES12]), but was first established by H. Wang and the second named author [BW16] via a different argument using the classification of approximate groups from [BGT11].

As before we consider three irreducible complex varieties $W_{1}, W_{2}, W_{3}$ of dimension $d$. We say that a subvariety $V \subset \prod_{i} W_{i}$ admits a power-saving $\eta>0$ if there exists $\tau \in \mathbb{N}$ such that

$$
\left|V \cap X_{1} \times X_{2} \times X_{3}\right| \leq O_{V, \tau}\left(N^{\operatorname{dim}(V)-\eta}\right) .
$$

for every $N \in \mathbb{N}$ and all finite subsets $X_{i} \subset W_{i}$ with $\left|X_{i}\right| \leq N^{d}$ and each $X_{i}$ in coarse $\tau$-general position in $W_{i}$.

Theorem 3.8. Suppose $V \subset W_{1} \times W_{2} \times W_{3}$ are irreducible complex varieties, and $\operatorname{dim}\left(W_{i}\right)=d$ and $\operatorname{dim}\left(\pi_{i j}(V)\right)=2 d=\operatorname{dim}(V)$ for all $i \neq j \in\{1,2,3\}$. Then either $V$ admits a power-saving $\frac{1}{16}$, or $V$ is in co-ordinatewise correspondence with the graph $\Gamma_{+} \subset G^{3}$ of the group operation of a commutative algebraic group $G$.

Remark 3.9. Note that we obtain a power saving which is independent of $d$. In fact the method gives a power-saving $\eta$ for any $\eta<\frac{d}{16 d-1}$, which is slightly better. For $d=1$, a power-saving of $\frac{1}{6}$ was obtained by Wang [Wan14] and independently by Raz, Sharir, and de Zeeuw [RSDZ16]. The method given below also gives $\frac{1}{6}$ when $d=1$, see Remark 3.14.

The remainder of this section is devoted to the proof of Theorem 3.8. As in the one-dimensional case, we first reformulate the result in the framework of coarse dimension using the notation of Section 2.

Theorem 3.10 (Reformulation of Theorem 3.8). Suppose $\bar{a}_{1}, \bar{a}_{2}, \bar{a}_{3} \in K^{d}$ with $\mathrm{d}^{0}\left(\bar{a}_{i}, \bar{a}_{j}\right)=$ $\mathrm{d}^{0}\left(\bar{a}_{1}, \bar{a}_{2}, \bar{a}_{3}\right)=2 d, \boldsymbol{\delta}\left(\bar{a}_{i}\right) \leq d$ and $\boldsymbol{\delta}\left(\bar{a}_{1}, \bar{a}_{2}, \bar{a}_{3}\right) \in\left[2 d-\frac{1}{16}, 2 d\right]$. Assume that each $\bar{a}_{i}$ is cgp in the sense of 3.0.3. Then there is an d-dimensional commutative algebraic group $G$ over $C_{0}$, and $\bar{a}_{1}^{\prime}, \bar{a}_{2}^{\prime}, \bar{a}_{3}^{\prime} \in G(K)$ such that $\mathrm{d}^{0}\left(\bar{a}_{i}^{\prime}\right)=d, \operatorname{acl}^{0}\left(\bar{a}_{i}\right)=\operatorname{acl}^{0}\left(\bar{a}_{i}^{\prime}\right)$ and $\bar{a}_{1}^{\prime}+\bar{a}_{2}^{\prime}=\bar{a}_{3}^{\prime}$.

Reduction of Theorem 3.8 to Theorem 3.10. This is essentially the same argument as in the one-dimensional case, so we shall be brief. Let $\eta=\frac{1}{16}$. Arguing by contradiction and carefully negating quantifiers we obtain an increasing sequence of integers $\left(N_{s}\right)_{s>0}$ and a sequence of finite sets $X_{i}^{s} \subset W_{i}(\mathbb{C})$ in coarse $s$-general position with $\left|X_{i}^{s}\right| \leq N_{s}^{d}$ but $\left|X_{1}^{s} \times X_{2}^{s} \times X_{3}^{s} \cap V\right| \geq N_{s}^{2 d-\eta}$. Passing to an ultralimit we obtain three internal sets $X_{i} \subset W_{i}(K)$, which we add as predicates to our language (thus turning them into definable sets). Clearly $\boldsymbol{\delta}\left(X_{i}\right) \leq d$ and $\boldsymbol{\delta}\left(X_{1} \times\right.$ $\left.X_{2} \times X_{3} \cap V\right) \geq 2 d-\eta$. By Fact 2.4 we find three tuples $\bar{a}_{i} \in X_{i}$ such that $\boldsymbol{\delta}\left(\bar{a}_{1}, \bar{a}_{2}, \bar{a}_{3}\right)=\boldsymbol{\delta}\left(X_{1} \times X_{2} \times X_{3} \cap V\right)$. By Lemma 3.6 each $\bar{a}_{i}$ is cgp. Moreover $\left(\bar{a}_{1}, \bar{a}_{2}, \bar{a}_{3}\right)$ is generic in $V$, for otherwise it would be contained in a subvariety $W \subsetneq V$ over $C_{0}$ forcing $\boldsymbol{\delta}\left(\bar{a}_{1}, \bar{a}_{2}, \bar{a}_{3}\right) \leq \boldsymbol{\delta}\left(X_{1} \times X_{2} \times X_{3} \cap W\right) \leq \operatorname{dim} W \leq 2 d-1$ (see Lemma 7.1). Therefore $\mathrm{d}^{0}\left(\bar{a}_{i}, \bar{a}_{j}\right)=\mathrm{d}^{0}\left(\bar{a}_{1}, \bar{a}_{2}, \bar{a}_{3}\right)=2 d$ and the assumptions of Theorem 3.10 are met.

Analogously to the one-dimensional case, the Szemerédi-Trotter bounds of Lemma 2.15 will be used to prove the following crucial step, which shows that the 2parameter family of varieties $\operatorname{loc}^{0}(\bar{a} / \bar{b})$ is in fact a 1 -parameter family. 
Lemma 3.11. Let $\eta \in\left[0, \frac{d}{8 d-1}\right)$. Let $\bar{x}_{1}, \ldots, \bar{x}_{4} \in K^{d}$, and set $\bar{a}=\left(\bar{x}_{1}, \bar{x}_{2}\right)$ and $\bar{b}=\left(\bar{x}_{3}, \bar{x}_{4}\right)$. Assume each $\bar{x}_{i}$ is cgp and $\boldsymbol{\delta}\left(\bar{x}_{i}\right) \leq \mathrm{d}^{0}\left(\bar{x}_{i}\right)=d$. Assume further $\boldsymbol{\delta}(\bar{a}, \bar{b}) \geq \mathrm{d}^{0}(\bar{a}, \bar{b})-\eta$ and $\mathrm{d}^{0}(\bar{a})=\mathrm{d}^{0}(\bar{b})=2 d$ and $\mathrm{d}^{0}(\bar{a} / \bar{b})=d$. Then there is $\bar{x}_{5} \in \operatorname{acl}^{0}(\bar{b})<\infty \cap \operatorname{acl}^{0}(\bar{a})^{<\infty}$ with $\mathrm{d}^{0}\left(\bar{x}_{5}\right)=\mathrm{d}^{0}\left(\bar{a} / \bar{x}_{5}\right)=d$.

Proof. First note that $\boldsymbol{\delta}(\bar{b}) \geq \mathrm{d}^{0}(\bar{b})-\eta$. To see this observe that by cgp either $\boldsymbol{\delta}\left(\bar{x}_{2} / \bar{b} \bar{x}_{1}\right)=0$ or $\boldsymbol{\delta}\left(\bar{x}_{1} / \bar{b}\right)=0$. Indeed otherwise $\mathrm{d}^{0}\left(\bar{x}_{2} / \bar{b}_{1}\right)=\mathrm{d}^{0}\left(\bar{x}_{2}\right)=d$ and $\mathrm{d}^{0}\left(\bar{x}_{1} / \bar{b}\right)=\mathrm{d}^{0}\left(\bar{x}_{1}\right)=d$, contradicting $\mathrm{d}^{0}\left(\bar{x}_{1}, \bar{x}_{2} / \bar{b}\right)=d$. So we have $\boldsymbol{\delta}(\bar{a} / \bar{b})=$ $\boldsymbol{\delta}\left(\bar{x}_{2} / \bar{b} \bar{x}_{1}\right)+\boldsymbol{\delta}\left(\bar{x}_{1} / \bar{b}\right) \leq d=\mathrm{d}^{0}(\bar{a} / \bar{b})$. And so $\boldsymbol{\delta}(\bar{b})=\boldsymbol{\delta}(\bar{a}, \bar{b})-\boldsymbol{\delta}(\bar{a} / \bar{b}) \geq 3 d-\eta-d=$ $\mathrm{d}^{0}(\bar{b})-\eta$.

Let $\bar{d} \in K^{<\infty}$ be a canonical base for $\bar{a}$ over $\bar{b}$ (see $\S 2.1 .12$ ). By definition $\bar{d} \in \operatorname{acl}^{0}(\bar{b})^{<\infty}$. We will show that this $\bar{x}_{5}:=\bar{d}$ satisfies the desired conclusion. By definition we have $\mathrm{d}^{0}(\bar{a} / \bar{d})=\mathrm{d}^{0}(\bar{a} / \bar{b})=d$. Hence $\mathrm{d}^{0}(\bar{d})=\mathrm{d}^{0}(\bar{a}, \bar{d})-\mathrm{d}^{0}(\bar{a} / \bar{d}) \geq$ $\mathrm{d}^{0}(\bar{a})-d=d$.

It only remains to show the upper bound $\mathrm{d}^{0}(\bar{d}) \leq d$. Indeed this will also imply that $\bar{d} \in \operatorname{acl}^{0}(\bar{a})^{<\infty}$, because then $\mathrm{d}^{0}(\bar{d})=d$ and $\mathrm{d}^{0}(\bar{a} \bar{d})=\mathrm{d}^{0}(\bar{a} / \bar{d})+\mathrm{d}^{0}(\bar{d})=2 d$, so $\mathrm{d}^{0}(\bar{a} \bar{d})=\mathrm{d}^{0}(\bar{a})$ and thus $\mathrm{d}^{0}(\bar{d} / \bar{a})=0$.

So suppose that $\mathrm{d}^{0}(\bar{d})>d$. Then $\mathrm{d}^{0}(\bar{b} / \bar{d})=\mathrm{d}^{0}(\bar{b})-\mathrm{d}^{0}(\bar{d})<d$. Writing

$$
\mathrm{d}^{0}(\bar{b} / \bar{d})=\mathrm{d}^{0}\left(\bar{x}_{3} / \bar{d}\right)+\mathrm{d}^{0}\left(\bar{x}_{4} / \bar{x}_{3}, \bar{d}\right)
$$

each term is $<d$. Since each $\bar{x}_{i}$ is cgp, this forces $\delta(\bar{b} / \bar{d})=0$. Consequently $\boldsymbol{\delta}(\bar{d})=\boldsymbol{\delta}(\overline{b d})$. But $\boldsymbol{\delta}(\bar{d} / \bar{b})=0$ by $(7)$. So $\boldsymbol{\delta}(\bar{d})=\boldsymbol{\delta}(\bar{b})$.

However $\boldsymbol{\delta}(\bar{b}) \geq \mathrm{d}^{0}(\bar{b})-\eta=2 d-\eta$. Using the Szemerédi-Trotter bound of Lemma 2.15 we will obtain an upper bound on $\boldsymbol{\delta}(\bar{d})$ contradicting this lower bound.

By the primitive element theorem, the field extension $C_{0}(\bar{d})$ is generated over $C_{0}$ by a tuple of length $\mathrm{d}^{0}(\bar{d})+1$. So we may assume $\bar{d} \in K^{\mathrm{d}^{0}(\bar{d})+1}$. Assume first that $\mathrm{d}^{0}(\bar{d})<2 d$, so $\max \left(2 d, \mathrm{~d}^{0}(\bar{d})+1\right)=2 d$; we handle the case $\mathrm{d}^{0}(\bar{d})=2 d$ separately below.

Now let $V=\operatorname{loc}^{0}(\bar{a} \bar{d}), X:=\left(X_{1} \times X_{2}\right) \cap V, X_{1}=\operatorname{tp}(\bar{a})(K)$ and $X_{2}=\operatorname{tp}(\bar{d})(K)$. Note that $X_{1} \subset K^{2 d}$ and $X_{2} \subset K^{\mathrm{d}^{0}(\bar{d})+1}$. First we check that $\boldsymbol{\delta}\left(X\left(\bar{d}_{1}\right) \cap X\left(\bar{d}_{2}\right)\right)=0$ when $\bar{d}_{1} \neq \bar{d}_{2}$ belong to $X_{2}$, so that $\beta=0$ in Lemma 2.15 .

Recall that $X\left(\bar{d}_{1}\right):=\left\{\bar{a}^{\prime} \in X_{1}: \bar{a}^{\prime} \bar{d}_{1} \in V\right\}$. By Fact 2.4 we may find $\bar{a}^{\prime}=$ $\bar{a}_{1}^{\prime} \bar{a}_{2}^{\prime} \in X\left(\bar{d}_{1}\right) \cap X\left(\bar{d}_{2}\right)$ such that $\boldsymbol{\delta}\left(\bar{a}^{\prime} / \bar{d}_{1} \bar{d}_{2}\right)=\boldsymbol{\delta}\left(X\left(\bar{d}_{1}\right) \cap X\left(\bar{d}_{2}\right)\right)$. Then $\bar{a}^{\prime} \bar{d}_{i} \in V$ for $i=1,2$. We are thus in the setting of Lemma 2.12. Since $\bar{d}_{1} \neq \bar{d}_{2}$ we conclude that $\mathrm{d}^{0}\left(\bar{a}^{\prime} / \bar{d}_{1} \bar{d}_{2}\right)<d$. Hence $\mathrm{d}^{0}\left(\bar{a}_{i}^{\prime} / \bar{d}_{1} \bar{d}_{2}\right)<d$, and since both $\bar{a}_{i}^{\prime}$ are cgp (because cgp is type invariant, see Remark 3.7), we conclude that $\boldsymbol{\delta}\left(\bar{a}_{i}^{\prime} / \bar{d}_{1} \bar{d}_{2}\right)=0$ for each $i$. Thus by additivity $\boldsymbol{\delta}\left(X\left(\bar{d}_{1}\right) \cap X\left(\bar{d}_{2}\right)\right)=\boldsymbol{\delta}\left(\bar{a}^{\prime} / \bar{d}_{1} \bar{d}_{2}\right)=0$ as claimed.

The Szemerédi-Trotter bound of Lemma 2.15 then gives the bound

$$
\boldsymbol{\delta}(X) \leq \max \left(\frac{1}{2} \boldsymbol{\delta}\left(X_{1}\right)+\boldsymbol{\delta}\left(X_{2}\right)-\epsilon_{0}\left(\boldsymbol{\delta}\left(X_{2}\right)-\frac{1}{2} \boldsymbol{\delta}\left(X_{1}\right)\right)^{+}, \boldsymbol{\delta}\left(X_{1}\right), \boldsymbol{\delta}\left(X_{2}\right)\right) ;
$$

using the incidence bound of $\left[\mathrm{FPS}^{+}\right.$17, Theorem 1.2] mentioned after Theorem 2.14, we obtain this bound for all $\epsilon_{0} \in\left[0, \frac{1}{8 d-1}\right)$. If the maximum on the right hand side is $\boldsymbol{\delta}\left(X_{1}\right)$, then $\boldsymbol{\delta}\left(X_{2}\right) \leq \frac{1}{2} \boldsymbol{\delta}\left(X_{1}\right) \leq d$, contradicting our lower bound $\boldsymbol{\delta}\left(X_{2}\right)=\boldsymbol{\delta}(\bar{d}) \geq$ $2 d-\eta$ above. If the maximum is $\boldsymbol{\delta}\left(X_{2}\right)$, then $\boldsymbol{\delta}(X)=\boldsymbol{\delta}(\bar{a} \bar{d}) \leq \boldsymbol{\delta}(\bar{d})$, so $\boldsymbol{\delta}(\bar{a} / \bar{d})=0$ and hence $\boldsymbol{\delta}(\bar{a} / \bar{b})=0$ by $(7)$. But $\boldsymbol{\delta}(\bar{a} / \bar{b})=\boldsymbol{\delta}(\bar{a} \bar{b})-\boldsymbol{\delta}(\bar{b}) \geq d-\eta>0$.

So we must conclude that

$$
\boldsymbol{\delta}(\bar{a} / \bar{b})+\boldsymbol{\delta}(\bar{d}) \leq \boldsymbol{\delta}(X) \leq \frac{1}{2} \boldsymbol{\delta}(\bar{a})+\boldsymbol{\delta}(\bar{d})-\epsilon_{0}\left(\boldsymbol{\delta}(\bar{d})-\frac{1}{2} \boldsymbol{\delta}(\bar{a})\right) .
$$

In other words, since $\boldsymbol{\delta}(\bar{d})=\boldsymbol{\delta}(\bar{b})$ : 


$$
\frac{1}{\epsilon_{0}} \boldsymbol{\delta}(\bar{a} \bar{b}) \leq \frac{1}{2} \boldsymbol{\delta}(\bar{a})\left(\frac{1}{\epsilon_{0}}+1\right)+\boldsymbol{\delta}(\bar{b})\left(\frac{1}{\epsilon_{0}}-1\right) .
$$
that

Finally since $\boldsymbol{\delta}(\bar{a}), \boldsymbol{\delta}(\bar{b}) \leq 2 d$ and $\boldsymbol{\delta}(\bar{a} \bar{b}) \geq 3 d-\eta$ by assumption, we conclude

$$
\eta \geq \frac{d}{8 d-1}
$$

a contradiction to our assumption.

We assumed above that $\mathrm{d}^{0}(\bar{d})<2 d$, and so we conclude from this contradiction that $\mathrm{d}^{0}(\bar{d})=d$ or $\mathrm{d}^{0}(\bar{d})=2 d$. it remains to rule out the latter case. If we were willing to weaken our bound to $\frac{d}{4(2 d+1)-1}$, the argument above would suffice. To obtain the sharper bound, we use an additional argument inspired by [RSDZ16, Theorem 4.3].

So assume $d^{0}(\bar{d})=2 d$, i.e. $\operatorname{acl}^{0}(\bar{d})=\operatorname{acl}^{0}(\bar{b})$.

Let $W:=\operatorname{loc}^{0}(\bar{a} \bar{b})$ and $V:=\operatorname{loc}^{0}(\bar{a} \bar{d})$. Write $V_{\bar{y}}$ for $\{\bar{x}:(\bar{x}, \bar{y}) \in V\}$, and similarly for $W_{\bar{y}}$. So $V_{\bar{d}}=\operatorname{loc}^{0}(\bar{a} / \bar{d})$ is the irreducible component of $W_{\bar{b}}=\operatorname{loc}^{0}(\bar{a} / \bar{b})$ containing $\bar{a}$. Now $\operatorname{tp}^{0}(\bar{d} / \bar{b})(K)$ is finite, so say $\operatorname{tp}^{0}(\bar{d} / \bar{b})(K)=\left\{\bar{d}_{1}, \ldots, \bar{d}_{k}\right\}$. Then $V_{\bar{d}_{i}}$ are precisely the irreducible components of $W_{\bar{b}}$, and $W_{\bar{b}}=\bigcup_{i} V_{\bar{d}_{i}}$. Indeed, any $V_{\bar{d}_{i}}$ is an automorphic image over $C_{0}(\bar{b})$ of $V_{\bar{d}}$ and so is a component, and conversely $\bigcup_{i} V_{\bar{d}_{i}}$ is automorphism-invariant over $C_{0}(\bar{b})$ and hence is defined over $C_{0}(\bar{b})$, so $W_{\bar{b}} \subset \bigcup_{i} V_{\bar{d}_{i}}$.

Claim 3.12. There are only finitely many $\bar{b}^{\prime} \in \operatorname{tp}^{0}(\bar{b})(K)$ with $\operatorname{dim}\left(W_{\bar{b}} \cap W_{\bar{b}^{\prime}}\right)=d$.

Proof. Fix field automorphisms $\tau_{i j} \in \operatorname{Aut}\left(K / C_{0}\right)$ such that $\tau_{i j}\left(\bar{d}_{i}\right)=\bar{d}_{j}$. Suppose $\bar{b}^{\prime} \in \operatorname{tp}^{0}(\bar{b})(K)$ and $\operatorname{dim}\left(W_{\bar{b}} \cap W_{\bar{b}^{\prime}}\right)=d$. Then $W_{\bar{b}}$ and $W_{\bar{b}^{\prime}}$ share a component, so say $V_{\bar{d}_{j}} \subset W_{\bar{b}^{\prime}}$. Now let $\sigma \in \operatorname{Aut}\left(K / C_{0}\right)$ be such that $\bar{b}^{\prime}=\sigma(\bar{b})$. Since the $\sigma\left(V_{\bar{d}_{i}}\right)$ are the irreducible components of $W_{\bar{b}^{\prime}}$, for some $i$ we have $\sigma\left(V_{\bar{d}_{i}}\right)=V_{\bar{d}_{j}}$.

Let $\sigma^{\prime}:=\tau_{i j}^{-1} \sigma$. Then $\sigma^{\prime}\left(V_{\bar{d}_{i}}\right)=\tau_{i j}^{-1} V_{\bar{d}_{j}}=V_{\tau_{i j}^{-1} \bar{d}_{j}}=V_{\bar{d}_{i}}$, so by the definition of canonical base, we have $\sigma^{\prime}\left(\bar{d}_{i}\right)=\bar{d}_{i}$. Then since $\bar{b} \in \operatorname{acl}^{0}\left(\bar{d}_{i}\right)$, there are only finitely many possibilities for $\sigma^{\prime}(\bar{b})$, and so there are only finitely many possibilities for $\bar{b}^{\prime}=\sigma(\bar{b})=\tau_{i j}\left(\sigma^{\prime}(\bar{b})\right)$.

Now let $G$ be the graph with vertex set $\operatorname{tp}(\bar{b})(K)$ and with an edge between $\bar{b}^{\prime}$ and $\bar{b}^{\prime \prime}$ if and only if $\operatorname{dim}\left(W_{\bar{b}^{\prime}} \cap W_{\bar{b}^{\prime \prime}}\right)=d$. By Claim 3.12, G has constant finite degree.

Claim 3.13. If $G=(A, E)$ is a graph where the vertex set $A$ is $\bigwedge$-internal and the edge relation $E$ is internal, and if $G$ has finite maximal degree $k$, then there is a $\bigwedge$-internal anticlique $A^{\prime}$ with $\boldsymbol{\delta}\left(A^{\prime}\right)=\boldsymbol{\delta}(A)$.

Proof. If $A$ is internal, then $G$ is the ultraproduct of finite graphs $G_{i}=\left(A_{i}, E_{i}\right)$ of maximal degree $k$. Then $G_{i}$ has chromatic number at most $k+1$, and so has an anticlique of size at least $\frac{\left|A_{i}\right|}{k+1}$. The ultraproduct of such anticliques is then an internal anticlique $A^{\prime} \subset A$ as required.

In general, our $\bigwedge$-internal $A$ is, by $\aleph_{1}$-compactness of the ultraproduct, contained in an internal $A_{0}$ such that $\left(A_{0}, E\right)$ has maximal degree at most $k$, because the property of having maximal degree at most $k$ can be expressed as the inconsistency of a partial $(k+1)$-type. So then the same holds for all internal $A_{1}$ with $A \subset A_{1} \subset$ $A_{0}$, and hence the claim follows from the internal case. 
Now let $X_{2} \subset \operatorname{tp}(\bar{b})(K)$ be an anticlique as in Claim 3.13 for the graph defined above, and $X_{1}:=\operatorname{tp}(\bar{a})(K)$ and $X:=\left(X_{1} \times X_{2}\right) \cap W$. If $\bar{a}^{\prime} \in X\left(\bar{b}_{1}\right) \cap X\left(\bar{b}_{2}\right)$ then $\mathrm{d}^{0}\left(\bar{a}^{\prime} / \bar{b}_{1} \bar{b}_{2}\right) \leq \operatorname{dim}\left(W_{\bar{b}_{1}} \cap W_{\bar{b}_{2}}\right)<d$ since $X_{2}$ is an anticlique, and so $\boldsymbol{\delta}\left(\bar{a}^{\prime}\right)=0$ by cgp. So we contradict the Szemerédi-Trotter bound exactly as in the case $d<\mathrm{d}^{0}(\bar{d})<2 d$ above.

This contradiction shows that $\mathrm{d}^{0}(\bar{d})=d$ and ends the proof.

Proof of Theorem 3.10. Here again the strategy is the same as in the 1-dimensional case, so we shall be brief. Let $\eta=\frac{1}{16}$.

As before set $X=\operatorname{tp}\left(\bar{a}_{2}, \bar{a}_{3} / \bar{a}_{1}\right)(K)$ and note that $\boldsymbol{\delta}\left(\bar{a}_{1}, \bar{a}_{2}, \bar{a}_{3}\right)=\boldsymbol{\delta}(X)+\boldsymbol{\delta}\left(\bar{a}_{1}\right)$ by additivity of $\boldsymbol{\delta}$. It follows that $\boldsymbol{\delta}(X) \geq d-\eta$.

By Fact 2.4 we may find $\left(\bar{a}_{4}, \bar{a}_{5}\right) \in X$ with $\boldsymbol{\delta}\left(a_{4}, a_{5} / a_{1}, a_{2}, a_{3}\right)=\boldsymbol{\delta}(X)$. Note that $\bar{a}_{4}, \bar{a}_{5}$ are both $c g p$ (see Remark 3.7). We will show that there are $\bar{a}_{6}$ and $\bar{a}_{7}$ in $K^{d}$ such that $\left(\bar{a}_{1}, \ldots, \bar{a}_{7}\right)$ satisfy the hypotheses of the abelian group configuration theorem as in the following diagram:

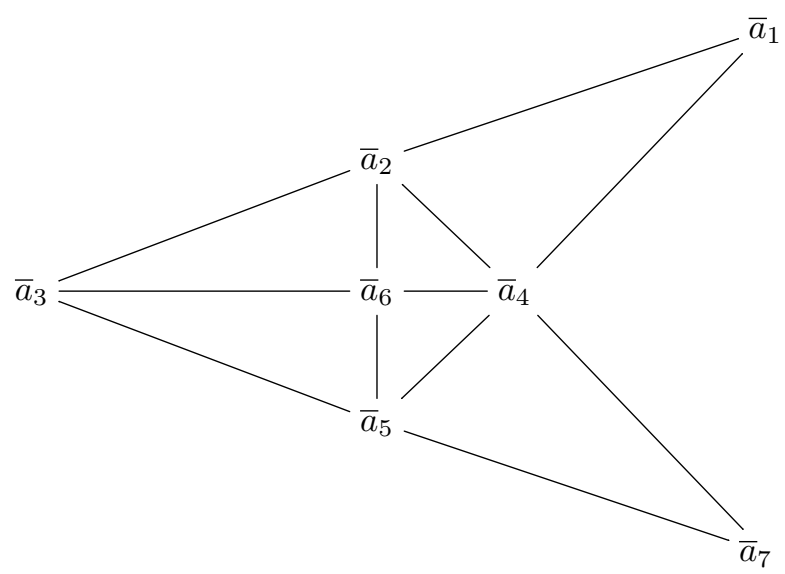

As earlier we have that $\mathrm{d}^{0}\left(\bar{a}_{4}, \bar{a}_{5}, \bar{a}_{1}\right)=2 d, \mathrm{~d}^{0}\left(\bar{a}_{4}, \bar{a}_{5}\right)=\mathrm{d}^{0}\left(\bar{a}_{4}, \bar{a}_{1}\right)=\mathrm{d}^{0}\left(\bar{a}_{5}, \bar{a}_{1}\right)=$ $2 d$. And we also have $\mathrm{d}^{0}\left(\bar{a}_{1}, \ldots, \bar{a}_{5}\right)=3 d$. Indeed otherwise $\mathrm{d}^{0}\left(\bar{a}_{4}, \bar{a}_{5} / \bar{a}_{1}, \bar{a}_{2}, \bar{a}_{3}\right)<$ $d$, which implies that $\mathrm{d}^{0}\left(\bar{a}_{i} / \bar{a}_{1}, \bar{a}_{2}, \bar{a}_{3}\right)<d$ for $i=4,5$ and thus $\boldsymbol{\delta}\left(\bar{a}_{i} / \bar{a}_{1}, \bar{a}_{2}, \bar{a}_{3}\right)=0$ since $\bar{a}_{4}, \bar{a}_{5}$ are cgp. But this contradicts $\boldsymbol{\delta}\left(\bar{a}_{4}, \bar{a}_{5} / \bar{a}_{1}, \bar{a}_{2}, \bar{a}_{3}\right)=\boldsymbol{\delta}(X)>0$.

Again as in the 1-dimensional case, using only the additivity of $\mathrm{d}^{0}$ we conclude that $\mathrm{d}^{0}\left(\bar{a}_{3}, \bar{a}_{4}\right)=\mathrm{d}^{0}\left(\bar{a}_{2}, \bar{a}_{5}\right)=2 d$ and $\mathrm{d}^{0}\left(\bar{a}_{2}, \bar{a}_{5} / \bar{a}_{3}, \bar{a}_{4}\right)=d$, and also that $\mathrm{d}^{0}\left(\bar{a}_{2}, \bar{a}_{4}\right)=\mathrm{d}^{0}\left(\bar{a}_{3}, \bar{a}_{5}\right)=2 d$ and $\mathrm{d}^{0}\left(\bar{a}_{3}, \bar{a}_{5} / \bar{a}_{2}, \bar{a}_{4}\right)=d$.

Moreover $\boldsymbol{\delta}\left(\bar{a}_{2}, \bar{a}_{3}\right)=\boldsymbol{\delta}\left(\bar{a}_{1}, \bar{a}_{2}, \bar{a}_{3}\right)$ by additivity since $\bar{a}_{1}$ is cgp. Similarly by additivity

$$
\boldsymbol{\delta}\left(\bar{a}_{2}, \bar{a}_{3}, \bar{a}_{4}, \bar{a}_{5}\right)=\boldsymbol{\delta}\left(\bar{a}_{1}, \ldots, \bar{a}_{5}\right)=\boldsymbol{\delta}(X)+\boldsymbol{\delta}\left(\bar{a}_{1}, \bar{a}_{2}, \bar{a}_{3}\right),
$$

hence this is $\geq 3 d-2 \eta$. Since $2 \eta \leq \frac{1}{8}<\frac{d}{8 d-1}$, we are thus in a position to apply Lemma 3.11 to both $\bar{a}_{2}, \bar{a}_{5}, \bar{a}_{3}, \bar{a}_{4}$ and to $\bar{a}_{2}, \bar{a}_{4}, \bar{a}_{3}, \bar{a}_{5}$. This yields $\bar{a}_{6}$ and $\bar{a}_{7}$ as desired and concludes the proof of the theorem.

Remark 3.14 (Quality of the power-saving). The quality of the power-saving depends crucially on the quality of $\epsilon_{0}$ in the Szemeredi-Trotter type bound of Lemma 2.15. We immediately lose a factor 2 because this bound is usually proven for real algebraic varities, while we consider complex varieties and somewhat carelessly view them as real varieties of twice the dimension. It is plausible that the bound $\epsilon_{0}=\frac{1}{2 n-1}$ holds in Theorem 2.14. In fact this is known when $n=1$ (see [SSZ18] or [RSDZ16, Thm 4.3]), and consequently Lemma 3.11 holds for all $\eta \in\left[0, \frac{1}{3}\right)$ when 
$d=1$ and thus yields a power-saving $\eta$ for any $\eta<\frac{1}{6}$ in the 1-dimensional ElekesSzabó theorem. This recovers the bound obtained in [Wan14] and [RSDZ16]. The latter work however gave more precise information on the multiplicative constant and the dependence on the degree of the variety $V$, which is an aspect we do not investigate in our paper (it would require working with the Hrushovski-Wagner fine pseudo-finite dimension while we restrict attention to the coarse dimension $\boldsymbol{\delta}$ ).

The following corollary indicates the robustness of the commutativity of the group in Theorem 3.8.

Corollary 3.15. Suppose $(G ; \cdot)$ is a connected complex algebraic group. Suppose the graph $\Gamma$ of multiplication admits no power-saving. Then $G$ is commutative.

Proof. By Theorem 3.8, $\Gamma$ is in co-ordinatewise correspondence with the graph $\Gamma_{+} \subset G^{\prime 3}$ of the group operation of a commutative connected algebraic group $G^{\prime}$. So this is an immediate consequence of Fact 2.13.

Remark 3.16. Another proof of this corollary was noted in [BW16]. It can be derived as a consequence of the Balog-Szemeredi-Gowers-Tao theorem combined with [BGT11, Theorem 2.5], one of the main results of [BGT11] which was proven there for linear algebraic groups, but can be extended to all algebraic groups.

In the following sections we will handle the general case of a cartesian product of an arbitrary number, say $n$, of subvarieties. As in the reformulations of ElekesSzabó's statements expounded above it is easy to see that a subvariety without power-saving leads to a tuple $\left(\bar{a}_{1}, \ldots, \bar{a}_{n}\right)$ such that each $\bar{a}_{i}$ is $c g p$, belongs to $K^{d}$ and has $\boldsymbol{\delta}\left(\bar{a}_{i}\right) \leq d$, such that

$$
\boldsymbol{\delta}\left(\bar{a}_{1}, \ldots, \bar{a}_{n}\right)=\mathrm{d}^{0}\left(\bar{a}_{1}, \ldots, \bar{a}_{n}\right) .
$$

In Sections 5 and 6 we will forget for a moment the original combinatorial problem and focus entirely on the study of these tuples. Then in Section 7 we will return to combinatorics and give a proof of Theorem 1.11 .

\section{NeCESSITy OF GENERAL POSITION}

We give an example showing that Theorem 3.8 fails dramatically if we weaken too far the coarse general position assumption in the definition of power-saving. Indeed varieties which are not even in correspondence with a group operation can then have no power-saving even when the finite sets are assumed to be say in weak general position, namely assuming $\boldsymbol{\delta}$ always drops when there is an algebraic dependence.

Define an operation $*: \mathbb{C}^{2} \times \mathbb{C}^{2} \rightarrow \mathbb{C}^{2}$ by

$$
\left(a_{1}, b_{1}\right) *\left(a_{2}, b_{2}\right)=\left(a_{1}+a_{2}+b_{1}^{2} b_{2}^{2}, b_{1}+b_{2}\right),
$$

and let $V:=\Gamma_{*}$ be its graph $\left\{(x, y, x * y) \mid x, y \in \mathbb{C}^{2}\right\}$.

Set $X_{N}:=\left\{0, \ldots, N^{4}-1\right\} \times\{0, \ldots, N-1\} \subset \mathbb{C}^{2}$. Then $\left|X_{N}^{3} \cap \Gamma_{*}\right| \geq \Omega\left(\left|X_{N}\right|^{2}\right)$; indeed, if $a_{i}<\frac{1}{3} N^{4}$ and $b_{i}<\frac{1}{2} N$ for $i=1,2$, then $\left(a_{1}, b_{1}\right) *\left(a_{2}, b_{2}\right) \in X_{N}$.

Now $\left|X_{N} \cap(\{0\} \times \mathbb{C})\right|=N=\left|X_{N}\right|^{\frac{1}{5}}$, so $X_{N}$ is not 6-cgp. Nevertheless $X_{N}$ is still in weak general position, namely $\left|X_{N} \cap W\right|=O_{W}\left(N^{4}\right)$ for every algebraic curve $W \leq \mathbb{C}^{2}$ and $N^{4} \leq\left|X_{N}\right|^{1-\frac{1}{5}}$. But if we were to remove the cgp assumption in the definition of power-saving, then $\left(X_{N}\right)_{N}$ witnesses that $\Gamma_{*}$ would admit no power-saving.

However, one can show that $\Gamma_{*}$ is not in co-ordinatewise correspondence with the graph of the group operation of any complex algebraic group $(G ; \cdot)$. We sketch a proof of this. 
Suppose such a group $(G ; \cdot)$ and a correspondence exist, defined over a finitely generated field $A \leq \mathbb{C}$. Then if we take independent generics $x_{1}, x_{2}, y_{1}, y_{2} \in \mathbb{C}^{2}$ over $A$ and set $z_{i j}:=x_{i} * y_{j}$, then $z_{22}$ lies in the algebraic closure $\operatorname{acl}\left(z_{11}, z_{12}, z_{21}\right)$ of $A\left(z_{11}, z_{12}, z_{21}\right)$. This follows from the equation in the algebraic group $G$

$$
x_{2}^{\prime} \cdot y_{2}^{\prime}=\left(x_{2}^{\prime} \cdot y_{1}^{\prime}\right)\left(x_{1}^{\prime} \cdot y_{1}^{\prime}\right)^{-1}\left(x_{1}^{\prime} \cdot y_{2}^{\prime}\right)
$$

cf. [HZ96, 6.2] and [Tao15, Theorem 41] where a converse to this is proven in the 1-dimensional case.

But if one takes $z_{11}, z_{12}, z_{21}, x_{2}$ independent generics and calculates in order $y_{1}, x_{1}, y_{2}, z_{22}$ using the definition of $*$, then $x_{1}, x_{2}, y_{1}, y_{2}$ are also independent generics, and writing $z_{11}=\left(z_{11}^{\prime}, z_{11}^{\prime \prime}\right)$ and so on, one obtains

$$
\begin{aligned}
z_{22}^{\prime} & =z_{21}^{\prime}+z_{12}^{\prime}-z_{11}^{\prime}-x_{2}^{\prime \prime 2}\left(z_{21}^{\prime \prime}-x_{2}^{\prime \prime}\right)^{2}+\left(z_{11}^{\prime \prime}-z_{21}^{\prime \prime}+x_{2}^{\prime \prime}\right)^{2}\left(z_{21}^{\prime \prime}-x_{2}^{\prime \prime}\right)^{2} \\
& -\left(z_{11}^{\prime \prime}-z_{21}^{\prime \prime}+x_{2}^{\prime \prime}\right)^{2}\left(z_{12}^{\prime \prime}-z_{11}^{\prime \prime}+z_{21}^{\prime \prime}-x_{2}^{\prime \prime}\right)^{2}, \\
z_{22}^{\prime \prime} & =z_{21}^{\prime \prime}+z_{12}^{\prime \prime}-z_{11}^{\prime \prime},
\end{aligned}
$$

and e.g. $x_{2}^{\prime \prime} z_{11}^{\prime \prime} z_{21}^{\prime \prime} z_{12}^{\prime \prime}$ has a non-zero coefficient, and so $z_{22}$ is not independent from $x_{2}$; but $z_{11}, z_{12}, z_{21}$ is independent from $x_{2}$ by assumption, so $z_{22} \notin \operatorname{acl}\left(z_{11}, z_{12}, z_{21}\right)$.

\section{Projective Geometries ARISING From VARIEties Without POWER-SAVing}

As hinted in Section 3, the proof of our main results will rely on the study of $c g p$ tuples from $K^{m}$ whose algebraic dimension $\mathrm{d}^{0}$ coincides with their pseudo-finite coarse dimension $\boldsymbol{\delta}$. In this section we study the geometry underlying these tuples, and prove Theorem 5.9 below, which establishes that the associated geometry is modular and hence satisfies the Veblen axiom making it, via the Veblen-Young co-ordinatisation theorem, a sum of projective geometries over division rings.

5.1. Geometries and modularity. We begin with recalling some classical terminology and basic results regarding abstract projective geometry (see [Art57, Cam92]) and the general notions of pregeometries, geometries and modularity.

Definition. A closure structure is a set $P$ with a map $\mathrm{cl}: \mathcal{P}(P) \rightarrow \mathcal{P}(P)$ such that for $A, B \subset P$ we have $A \subset \operatorname{cl}(A), A \subset B \Rightarrow \operatorname{cl}(A) \subset \operatorname{cl}(B)$, and $\operatorname{cl}(\operatorname{cl}(A))=$ $\operatorname{cl}(A)$. A subset of $P$ is closed if it is in the image of cl. A closure structure $(P, \mathrm{cl})$ is a pregeometry if the following two properties also hold:

- Exchange: $b \in \operatorname{cl}(A \cup\{c\}) \backslash \operatorname{cl}(A) \Rightarrow c \in \operatorname{cl}(A \cup\{b\})$;

- Finite character: $\operatorname{cl}(A)=\bigcup_{A_{0} \subset A, A_{0} \text { finite }} \operatorname{cl}\left(A_{0}\right)$.

Finite pregeometries are also known as matroids.

Let $(P, \mathrm{cl})$ be a pregeometry. For $A, B \subset P$, a basis for $A$ over $B$ is a subset $A^{\prime} \subset A$ of minimal size such that $\operatorname{cl}\left(A^{\prime} \cup B\right)=\operatorname{cl}(A \cup B)$. Any two bases have the same cardinality, which is denoted by $\operatorname{dim}(A / B)$ and called the dimension of $A$ over $B$. When $B$ is empty, this is the dimension of $A$, which we denote as usual by $\operatorname{dim}(A)$.

Subsets $A, B \subset P$ are independent over a subset $C \subset P$, written $A \downarrow_{C} B$, if $\operatorname{dim}\left(A^{\prime} / B \cup C\right)=\operatorname{dim}\left(A^{\prime} / C\right)$ for any finite $A^{\prime} \subset A$.

A pregeometry $(P, \mathrm{cl})$ is a geometry if $\operatorname{cl}(\emptyset)=\emptyset$ and $\operatorname{cl}(\{a\})=\{a\}$ for $a \in P$. Every pregeometry gives rise to a geometry by projectivisation: the projectivisation of a closure structure $(P, \mathrm{cl})$ is the closure structure $\mathbb{P}(P, \mathrm{cl})$ with points $\{\operatorname{cl}(\{a\}): a \notin \operatorname{cl}(\emptyset)\}$ and the induced closure. If $(P, \mathrm{cl})$ is a pregeometry, then $\mathbb{P}(P, \mathrm{cl})$ is the associated geometry.

A geometry $(P, \mathrm{cl})$ is said to be modular if for distinct $a_{1}, a_{2} \in P$ and $B \subset P$, and if $a_{2} \in \operatorname{cl}\left(\left\{a_{1}\right\} \cup B\right) \backslash \operatorname{cl}(B)$, then there exists $d \in \operatorname{cl}(B)$ such that $d \in \operatorname{cl}\left(\left\{a_{1}, a_{2}\right\}\right)$. 
A pregeometry is modular if its associated geometry is modular. Equivalently ([Mar02, Lemma 8.1.13]), for any closed sets $A, B$,

$$
\operatorname{dim}(A \cup B)=\operatorname{dim}(A)+\operatorname{dim}(B)-\operatorname{dim}(A \cap B) .
$$

Points $a_{1}, a_{2}$ of a geometry $(P, \mathrm{cl})$ are non-orthogonal if there exists $B \subset P$ such that $a_{2} \in \operatorname{cl}\left(\left\{a_{1}\right\} \cup B\right) \backslash \operatorname{cl}(B)$.

A subgeometry of a geometry $(P, \mathrm{cl})$ is the restriction $\left(Y, \mathrm{cl}\left\lceil_{Y}\right)\right.$ to a subset $Y \subset P$, where $\left.\operatorname{cl}\right|_{Y}(A):=\operatorname{cl}(A) \cap Y$.

The sum of geometries $\left(P_{i}, \mathrm{cl}_{i}\right)$ is the non-interacting geometry on the disjoint union, namely the geometry $\left(\dot{\bigcup}_{i} P_{i}, \mathrm{cl}\right)$ where $\operatorname{cl}\left(\dot{\bigcup}_{i} A_{i}\right):=\dot{\bigcup}_{i} \operatorname{cl}_{i}\left(A_{i}\right)$.

The proofs of all claims made in the above definitions are straightforward and classical. We refer the reader to [TZ12, Appendix C.1] for them and further details.

Example 5.1 (Projective spaces over division rings). If $V$ is a vector space over a division ring $F$, then $V$ equipped with $F$-linear span forms a pregeometry $\left(V,\langle\cdot\rangle_{F}\right)$ of $\operatorname{dimension} \operatorname{dim}(V)$, and the associated geometry $\mathbb{P}(V):=\mathbb{P}\left(V,\langle\cdot\rangle_{F}\right)$ is the projective space of $V$; it also has dimension $\operatorname{dim}(V)$, and it is a modular geometry.

Example 5.2 (Algebraic closure). An algebraically closed field $K$ equipped with field-theoretic algebraic closure over the prime field forms a pregeometry $(K$, acl $)$. The dimension is the transcendence degree over the prime field. If $\operatorname{dim}(K) \geq 3$ then the associated geometry is not modular, as can be seen by considering a generic solution to $b=c_{1} a+c_{2}$, see [TZ12, Appendix C.1].

Example 5.3 (Algebraic closure on tuples). If $C_{0} \leq K$ are algebraically closed fields, the set of all tuples $K^{<\infty}$ equipped with the algebraic closure acl ${ }^{0}$ over $C_{0}$ forms a closure structure, where the closure of a subset $A \subset K^{<\infty}$ is $\operatorname{acl}^{0}(A)^{<\infty}$ as defined in (6). But it is in general not a pregeometry.

In the sequel we will only consider closure operators of the types described in the above examples.

Let $(P, \mathrm{cl})$ be a modular geometry. Then $a, b \in P$ are non-orthogonal if and only if there exists $c \in P \backslash\{a\}$ such that $a \in \operatorname{cl}(\{b, c\})$. In other words, $a=b$ or $|\operatorname{cl}(\{a, b\})|>2$. It is easy to see from modularity that this is an equivalence relation.

Example 5.4 (Abstract projective space). An abstract projective space is a pair $(P, L)$ of sets, where $P$ is the set of points and $L$ the set of lines, a unique line passes through every two distinct points, every line has at least three points and the Veblen axiom holds: given four distinct points $a, b, c, d$, if the lines $a b$ and $d c$ intersect, then so do $a d$ and $b c$. Any such abstract projective space gives rise to a modular geometry on $P$ in which the closure of a subset is the union of all lines passing through two points in the subset. Conversely any modular geometry in which every pair of points is non-orthogonal gives rise to an abstract projective space with the same set of points and with the set of lines being the set of closures of pairs of distinct points.

We now recall the classical Veblen-Young co-ordinatisation theorem of projective geometry, which characterises modular geometries.

Fact 5.5. If $(P, \mathrm{cl})$ is a modular geometry, and every two points $a, b \in P$ are nonorthogonal, and $\operatorname{dim}(P) \geq 4$, then $P$ is isomorphic to a projective space $\mathbb{P}(V)$, where $V$ is a vector space over a division ring.

More generally, if $(P, \mathrm{cl})$ is a modular geometry, then non-orthogonality is an equivalence relation, and $(P, \mathrm{cl})$ is the sum of the subgeometries on its non-orthogonality classes, each of which either has dimension $\leq 2$, or is a projective space over a division ring, or is a non-Desarguesian projective plane. 
Proof. This is a consequence of the classical Veblen-Young co-ordinatisation theorem for projective geometries. Veblen's axiom is a direct consequence of modularity. We refer to [Cam92, Theorem 3.6] for a statement which directly implies the stated result and for an overview of the proof, and to [Art57, Chapter II] for a detailed proof of the co-ordinatisation theorem for Desarguesian projective planes.

In our applications the geometries will be modular and infinite dimensional. So by the above they will be sums of projective geometries over division rings.

5.2. Coarse general position, coherence and modularity. We recall the notion of coarse general position for tuples introduced in §3.0.3. We keep the notation and setup of Section 2.

Definition 5.6. A tuple $\bar{c} \in K^{<\infty}$ is said to be in coarse general position (or is cgp) if for any $B \subset K^{<\infty}$, if $\bar{c} \not^{0} B$ then $\boldsymbol{\delta}(\bar{c} / B)=0$.

Recall that $K^{<\infty}$ is the set of all tuples of elements from $K$ and $c \chi^{0} B$ means that $\mathrm{d}^{0}(\bar{c} / B)<\mathrm{d}^{0}(\bar{c})$, where $\mathrm{d}^{0}(\bar{c} / B)$ denotes as earlier the transcendence degree of the tuple $\bar{c}$ over the field $C_{0}(B)$ field generated by all co-ordinates of elements from $B$, where $C_{0} \leq K$ is the base field defined in 2.1.7. The coarse dimension $\delta$ was defined in (3).

Definition 5.7. A subset $P \subset K^{<\infty}$ is said to be cgp-coherent if every $\bar{a} \in P$ is cgp and $\boldsymbol{\delta}\left(\bar{a}_{1}, \ldots, \bar{a}_{n}\right)=\mathrm{d}^{0}\left(\bar{a}_{1}, \ldots, \bar{a}_{n}\right)$ for all choices of $\bar{a}_{1}, \ldots, \bar{a}_{n} \in P$.

In this paper, we abbreviate 'cgp-coherent' to just 'coherent'

We will also say that a tuple of tuples from $K^{<\infty}$ is coherent when the set of its elements is coherent.

Remark 5.8. The term "coherent" is borrowed from [Hru13, Section 5], where it is used in a parallel context to refer to the same idea that a pseudo-finite dimension notion is in accord with transcendence degree.

We are now ready to state the main result of this section.

Theorem 5.9. Suppose $P \subset K^{<\infty}$ is coherent. Then $\left(P ; \operatorname{acl}^{0} \uparrow_{P}\right)$ is a pregeometry. Moreover $P$ extends to a coherent $P^{\prime} \subset K^{<\infty}$ such that the geometry $\mathcal{G}_{P^{\prime}}:=\mathbb{P}\left(P^{\prime} ; \operatorname{acl}^{0} \uparrow_{P^{\prime}}\right)$ is a sum of 1 -dimensional geometries and infinite dimensional projective geometries over division rings.

Here the closure operator is simply the restriction to $P^{\prime}$ of the algebraic closure $\operatorname{acl}^{0}$ as in Example 5.3, namely if $A \subset P^{\prime}, \operatorname{acl}^{0}\left\lceil P^{\prime}(A)\right.$ is the set of tuples in $P^{\prime}$ whose co-ordinates are algebraic over the subfield of $K$ generated by $C_{0}$ and the set of all co-ordinates of all tuples from $A$.

The proof of Theorem 5.9 will proceed in two steps. First we will show that if $P \subset$ $K^{<\infty}$ is coherent, then its coherent algebraic closure $\operatorname{ccl}(P):=\left\{\bar{x} \in \operatorname{acl}^{0}(P)^{<\infty}\right.$ : $\bar{x}$ is cgp and $\left.\boldsymbol{\delta}(\bar{x})=\mathrm{d}^{0}(\bar{x})\right\}$ is also coherent. And second we will prove that if $P=\operatorname{ccl}(P)$ is coherent, then $\left(P ; \operatorname{acl}^{0} \uparrow_{P}\right)$ is a modular pregeometry. The latter step will use the incidence bounds à la Szemerédi-Trotter recalled in Section 2.2. Theorem 5.9 will then follow by applying the Veblen-Young theorem recalled in Fact 5.5 above to the projectivisation of $\left(P ; \operatorname{acl}^{0} \uparrow_{P}\right)$.

The rest of this section is devoted to the proof of Theorem 5.9.

\subsubsection{Properties of coherent sets.}

Proposition 5.10. If $P \subset K^{\infty}$ is coherent then $\left(P ; \operatorname{acl}^{0} \uparrow_{P}\right)$ is a pregeometry.

Proof. We verify exchange, the other properties being immediate. Suppose $\bar{b} \in$ $\operatorname{acl}^{0}(A \cup\{\bar{c}\}) \backslash \operatorname{acl}^{0}(A)$ for $A \subset P$ and $\bar{b}, \bar{c} \in P$. So $\bar{b} \mathbb{L}_{A}^{0} \bar{c}$. By symmetry of $\downarrow^{0}$ (see $\S 2.1 .7)$, we get $\bar{c} \chi_{A}^{0} \bar{b}$. Now the next lemma forces $\bar{c} \in \operatorname{acl}^{0}(A \cup\{\bar{b}\})$. 
Lemma 5.11. Suppose $P \subset K^{<\infty}$ is coherent. Let $\bar{c} \in P$ and $A, B \subset P$. Then either $\bar{c} \downarrow_{A}^{0} B$, or $\bar{c} \in \operatorname{acl}^{0}(A \cup B)$.

Proof. Suppose $\bar{c} \chi_{A}^{0} B$. Then in particular $\bar{c} \mathfrak{X}^{0}(B \cup A)$, and so $\bar{c} \mathfrak{X}^{0} \bar{b} \bar{a}$ for some tuples $\bar{b} \in B^{<\infty}$ and $\bar{a} \in A^{<\infty}$. Since $\bar{c}$ is cgp this implies $\boldsymbol{\delta}(\bar{c} / \bar{b} \bar{a})=0$. Since $P$ is coherent, $\mathrm{d}^{0}(\bar{c} \bar{b} \bar{a})=\boldsymbol{\delta}(\bar{c} \bar{b} \bar{a})$ and $\mathrm{d}^{0}(\bar{b} \bar{a})=\boldsymbol{\delta}(\bar{b} \bar{a})$ so by additivity of $\mathrm{d}^{0}$ and $\boldsymbol{\delta}$ we get $\mathrm{d}^{0}(\bar{c} / \bar{b} \bar{a})=0$. Hence $\bar{c} \in \operatorname{acl}^{0}(A \cup B)$.

The next lemma will be used to form coherent sets.

Lemma 5.12. Let $\bar{a}_{1}, \ldots, \bar{a}_{n} \in K^{<\infty}$. Assume that each $\bar{a}_{i}$ is cgp and $\boldsymbol{\delta}\left(\bar{a}_{i}\right) \leq$ $\mathrm{d}^{0}\left(\bar{a}_{i}\right)$. Then for every $C \subset K^{<\infty}$ we have:

$$
\boldsymbol{\delta}\left(\bar{a}_{1}, \ldots, \bar{a}_{n} / C\right) \leq \mathrm{d}^{0}\left(\bar{a}_{1}, \ldots, \bar{a}_{n} / C\right) .
$$

Moreover $\boldsymbol{\delta}\left(\bar{a}_{1}, \ldots, \bar{a}_{n}\right)=\mathrm{d}^{0}\left(\bar{a}_{1}, \ldots, \bar{a}_{n}\right)$ if and only if $\left\{\bar{a}_{1}, \ldots, \bar{a}_{n}\right\}$ is coherent.

Proof. The proof is by induction on $n$. Suppose first $n=1$. We have a cgp $\bar{a}_{1} \in K^{<\infty}$ such that $\boldsymbol{\delta}\left(\bar{a}_{1}\right) \leq \mathrm{d}^{0}\left(\bar{a}_{1}\right)$ and we need to show that $\boldsymbol{\delta}\left(\bar{a}_{1} / C\right) \leq \mathrm{d}^{0}\left(\bar{a}_{1} / C\right)$. If $\bar{a}_{1} \mathfrak{L}^{0} C$, then $\mathrm{d}^{0}\left(\bar{a}_{1} / C\right)=\mathrm{d}^{0}\left(\bar{a}_{1}\right)$, so the desired inequality follows immediately. On the other hand, if $\bar{a}_{1} \mathfrak{X}^{0} C$, then by $\operatorname{cgp} \boldsymbol{\delta}\left(\bar{a}_{1} / C\right)=0$, so the desired inequality is then obvious.

Suppose (11) holds for $n-1$ tuples and any $C$. Let $\bar{x}=\bar{a}_{1} \ldots \bar{a}_{n-1}$.

$\boldsymbol{\delta}\left(\overline{x a}_{n} / C\right)=\boldsymbol{\delta}\left(\bar{x} / C \cup\left\{\bar{a}_{n}\right\}\right)+\boldsymbol{\delta}\left(\bar{a}_{n} / C\right) \leq \mathrm{d}^{0}\left(\bar{x} / C \cup\left\{\bar{a}_{n}\right\}\right)+\mathrm{d}^{0}\left(\bar{a}_{n} / C\right)=\mathrm{d}^{0}\left(\overline{x a}_{n} / C\right)$,

where we applied the induction hypothesis and the case $n=1$.

Finally we turn to the last claim of the lemma. Suppose $\delta\left(\bar{a}_{1} \ldots \bar{a}_{n}\right)=\mathrm{d}^{0}\left(\bar{a}_{1} \ldots \bar{a}_{n}\right)$. We need to show that $\boldsymbol{\delta}(\bar{x})=\mathrm{d}^{0}(\bar{x})$ for all concatenated tuples $\bar{x}$ made of subtuples from $\left\{\bar{a}_{1}, \ldots, \bar{a}_{n}\right\}$. Note that for every tuple of $\bar{a}_{i}$ 's the quantities $\boldsymbol{\delta}$ and $\mathrm{d}^{0}$ depend only on the subset of $\bar{a}_{i}$ 's appearing in the tuple (see Fact 2.5), so up to relabelling co-ordinates we may assume that $\bar{x}=\bar{a}_{1} \ldots \bar{a}_{i}$ for $i \in[1, n]$. Let $\bar{y}:=\bar{a}_{i+1} \ldots \bar{a}_{n}$. Then by assumption $\boldsymbol{\delta}(\overline{x y})=\mathrm{d}^{0}(\overline{x y})$. By (11) we have $\boldsymbol{\delta}(\bar{y} / \bar{x}) \leq \mathrm{d}^{0}(\bar{y} / \bar{x})$ and $\boldsymbol{\delta}(\bar{x}) \leq \mathrm{d}^{0}(\bar{x})$. Hence by additivity we conclude that the last two inequalities are equalities. This ends the proof.

Finally we record one last observation, which will be useful in the next paragraph.

Lemma 5.13. If $P \subset K^{<\infty}$ is coherent and $\bar{x} \in \operatorname{acl}^{0}(P)^{<\infty}$. Then $\boldsymbol{\delta}(\bar{x}) \geq \mathrm{d}^{0}(\bar{x})$.

Proof. Pick $\bar{a}_{1}, \ldots, \bar{a}_{n} \in P$ such that $\bar{x} \in \operatorname{acl}^{0}\left(\left\{\bar{a}_{1}, \ldots, \bar{a}_{n}\right\}\right)^{<\infty}$ and concatenate the $\bar{a}_{i}$ 's in $\bar{a}:=\bar{a}_{1} \ldots \bar{a}_{n}$. Then $\mathrm{d}^{0}(\bar{a})=\mathrm{d}^{0}(\overline{a x})$. By additivity $\mathrm{d}^{0}(\overline{a x})=\mathrm{d}^{0}(\bar{x})+\mathrm{d}^{0}(\bar{a} / \bar{x})$ and $\boldsymbol{\delta}(\overline{a x})=\boldsymbol{\delta}(\bar{x})+\boldsymbol{\delta}(\bar{a} / \bar{x})$. By coherence of $P$ we have $\boldsymbol{\delta}(\bar{a})=\mathrm{d}^{0}(\bar{a})$. But $\boldsymbol{\delta}(\bar{a} / \bar{x}) \leq \mathrm{d}^{0}(\bar{a} / \bar{x})$ by Lemma 5.12 . So

$$
\boldsymbol{\delta}(\bar{x}) \geq \boldsymbol{\delta}(\overline{a x})-\mathrm{d}^{0}(\bar{a} / \bar{x}) \geq \boldsymbol{\delta}(\bar{a})-\mathrm{d}^{0}(\bar{a} / \bar{x})=\mathrm{d}^{0}(\bar{a})-\mathrm{d}^{0}(\bar{a} / \bar{x})=\mathrm{d}^{0}(\bar{x}) .
$$

5.2.2. The Veblen axiom and incidence bounds. In this paragraph we exploit the Szemerédi-Trotter-type bounds described in Subsection 2.2 in order to show that the pregeometry $\left(P ; \operatorname{acl}^{0} \uparrow_{P}\right)$ satisfies the Veblen axiom of projective geometry.

Proposition 5.14. Assume $P \subset K^{<\infty}$ is coherent, let $\bar{a}_{1}, \bar{a}_{2} \in P$ and $B \subset P$. Assume that $\bar{a}_{1}, \bar{a}_{2} \notin \operatorname{acl}^{0}(B)^{<\infty}$, but $\bar{a}_{2} \in \operatorname{acl}^{0}\left(\left\{\bar{a}_{1}\right\} \cup B\right)^{<\infty} \backslash \operatorname{acl}^{0}\left(\bar{a}_{1}\right)^{<\infty}$. Then there is $\bar{d} \in \operatorname{acl}^{0}(B)^{<\infty}$ such that $\bar{d} \in \operatorname{acl}^{0}\left(\bar{a}_{1}, \bar{a}_{2}\right)^{<\infty}$ and $\boldsymbol{\delta}(\bar{d})=\mathrm{d}^{0}(\bar{d})=\mathrm{d}^{0}\left(\bar{a}_{1}\right)=$ $\mathrm{d}^{0}\left(\bar{a}_{2}\right)$. 
Proof. For brevity set $\bar{a}:=\bar{a}_{1} \bar{a}_{2}$. Without loss of generality we may assume $B$ is finite and $B=\left\{\bar{a}_{3}, \ldots, \bar{a}_{n}\right\}$. We set $\bar{b}=\left(\bar{a}_{3}, \ldots, \bar{a}_{n}\right)$. First we check that $\bar{a}_{1} \in \operatorname{acl}^{0}\left(\bar{a}_{2}, \bar{b}\right)<\infty, \bar{a}_{i} \downarrow^{0} \bar{b}$ and $\bar{a}_{1} \mathfrak{L}^{0} \bar{a}_{2}$, and that if $k:=\mathrm{d}^{0}\left(\bar{a}_{1}\right)$, then $\mathrm{d}^{0}\left(\bar{a}_{2}\right)=$ $k$ and $\operatorname{dim}(\bar{a})=2 k$. The first property follows from the exchange property of pregeometries and from Proposition 5.10. Lemma 5.11 tells us that $\bar{a}_{i} \bigsqcup^{0} \bar{b}$, since $\bar{a}_{i} \notin \operatorname{acl}^{0}(\bar{b})<\infty$. For the same reason $\bar{a}_{1} \downarrow^{0} \bar{a}_{2}$. Then $\mathrm{d}^{0}(\bar{a} \bar{b})=\mathrm{d}^{0}\left(\bar{a}_{1} \bar{b}\right)=\mathrm{d}^{0}\left(\bar{a}_{2} \bar{b}\right)$ is equal to both $\mathrm{d}^{0}\left(\bar{a}_{1}\right)+\mathrm{d}^{0}(\bar{b})$ and $\mathrm{d}^{0}\left(\bar{a}_{2}\right)+\mathrm{d}^{0}(\bar{b})$. Hence $\mathrm{d}^{0}\left(\bar{a}_{2}\right)=k$ and $\mathrm{d}^{0}(\bar{a})=2 k$. This also shows that $\mathrm{d}^{0}(\bar{a} / \bar{b})=k$.

Let $\bar{d} \in K^{<\infty}$ be a canonical base for $\bar{a}$ over $\bar{b}$ (see $\S 2.1 .12$ ). By definition $\bar{d} \in$ $\operatorname{acl}^{0}(\bar{b})^{<\infty}$. We will show that this $\bar{d}$ satisfies the desired conclusion. By definition we have $\mathrm{d}^{0}(\bar{a} / \bar{d})=\mathrm{d}^{0}(\bar{a} / \bar{b})=k$. Hence $\mathrm{d}^{0}(\bar{d})=\mathrm{d}^{0}(\bar{a} \bar{d})-\mathrm{d}^{0}(\bar{a} / \bar{d}) \geq \mathrm{d}^{0}(\bar{a})-k=k$. By Lemma $5.13 \boldsymbol{\delta}(\bar{d}) \geq \mathrm{d}^{0}(\bar{d})$. Hence we are left to show the upper bound $\boldsymbol{\delta}(\bar{d}) \leq k$.

To this end let $V$ be the locus of the tuple $\bar{a} \bar{d}$, i.e. $V=\operatorname{loc}^{0}(\bar{a}, \bar{d})$, let $X_{1} \subset$ $K^{<\infty}$ be the type of $\bar{a}$, i.e. $X_{1}=\operatorname{tp}(\bar{a})(K)$, let $X_{2}=\operatorname{tp}(\bar{d})(K)$ and finally let $X=\left(X_{1} \times X_{2}\right) \cap V$. We wish to apply the Szemerédi-Trotter bound of Lemma 2.15 to this data.

For this we first show that $\boldsymbol{\delta}\left(X\left(\bar{d}_{1}\right) \cap X\left(\bar{d}_{2}\right)\right)=0$ for all $\bar{d}_{1}, \bar{d}_{2} \in X_{2}$ with $\bar{d}_{1} \neq \bar{d}_{2}$, so that $\beta=0$ in this lemma. Recall that $X\left(\bar{d}_{1}\right):=\left\{\bar{a}^{\prime} \in X_{1}: \bar{a}^{\prime} \bar{d}_{1} \in V\right\}$. By Fact 2.4 we may find $\bar{a}^{\prime}=\bar{a}_{1}^{\prime} \bar{a}_{2}^{\prime} \in X\left(\bar{d}_{1}\right) \cap X\left(\bar{d}_{2}\right)$ such that $\boldsymbol{\delta}\left(\bar{a}^{\prime} / \bar{d}_{1} \bar{d}_{2}\right)=\boldsymbol{\delta}\left(X\left(\bar{d}_{1}\right) \cap\right.$ $\left.X\left(\bar{d}_{2}\right)\right)$. Then $\bar{a}^{\prime} \bar{d}_{i} \in V$ for $i=1,2$. We are thus in the setting of Lemma 2.12. Since $\bar{d}_{1} \neq \bar{d}_{2}$ we conclude that $\mathrm{d}^{0}\left(\bar{a}^{\prime} / \bar{d}_{1} \bar{d}_{2}\right)<k$. Hence $\mathrm{d}^{0}\left(\bar{a}_{i}^{\prime} / \bar{d}_{1} \bar{d}_{2}\right)<k$, and since both $\bar{a}_{i}^{\prime}$ are cgp (because cgp is type invariant, see Remark 3.7), we conclude that $\boldsymbol{\delta}\left(\bar{a}_{i}^{\prime} / \bar{d}_{1} \bar{d}_{2}\right)=0$ for each $i$. Thus by additivity $\boldsymbol{\delta}\left(X\left(\bar{d}_{1}\right) \cap X\left(\bar{d}_{2}\right)\right)=\boldsymbol{\delta}\left(\bar{a}^{\prime} / \bar{d}_{1} \bar{d}_{2}\right)=0$ as claimed.

Next note that $\boldsymbol{\delta}\left(X_{2}\right)=\boldsymbol{\delta}(\bar{d}), \boldsymbol{\delta}\left(X_{1}\right)=\boldsymbol{\delta}(\bar{a})=\mathrm{d}^{0}(\bar{a})=2 k$ by coherence, and $\boldsymbol{\delta}(X) \geq \boldsymbol{\delta}(\bar{a} \bar{d})=\boldsymbol{\delta}(\bar{a} / \bar{d})+\boldsymbol{\delta}(\bar{d})$. But $\boldsymbol{\delta}(\bar{a} / \bar{d}) \geq \boldsymbol{\delta}\left(\bar{a} / \operatorname{acl}^{0}(\bar{b})^{<\infty}\right)=\boldsymbol{\delta}(\bar{a} / \bar{b})$ by $(7)$. By coherence $\boldsymbol{\delta}(\bar{a} / \bar{b})=\mathrm{d}^{0}(\bar{a} / \bar{b})=k$. We conclude

$$
\boldsymbol{\delta}(X) \geq k+\boldsymbol{\delta}(\bar{d})=\frac{1}{2} \boldsymbol{\delta}\left(X_{1}\right)+\boldsymbol{\delta}\left(X_{2}\right) .
$$

Now comparing this to the Szemerédi-Trotter bound of Lemma 2.15 we obtain $\boldsymbol{\delta}\left(X_{2}\right) \leq \frac{1}{2} \boldsymbol{\delta}\left(X_{1}\right)$. In other words $\boldsymbol{\delta}(\bar{d}) \leq k$. This ends the proof.

5.2.3. Proof of Theorem 5.9. Here we show Theorem 5.9. Lemma 5.14 will help us find a modular geometry explaining algebraic dependence on a coherent set. This is the engine behind our main results. The idea comes from [Hru13, Subsection 5.17], and the context is essentially that of [Hru13, Remark 5.26].

Definition 5.15. For $P \subset K^{<\infty}$, define the coherent algebraic closure by

$$
\operatorname{ccl}(P):=\left\{\bar{a} \in \operatorname{acl}^{0}(P)^{<\infty}: \bar{a} \text { is cgp and } \boldsymbol{\delta}(\bar{a})=\mathrm{d}^{0}(\bar{a})\right\} .
$$

Lemma 5.16. If $P$ is coherent, then so is $\operatorname{ccl}(P)$.

Proof. We need to show that $\boldsymbol{\delta}\left(\bar{a}_{1} \ldots \bar{a}_{n}\right)=\mathrm{d}^{0}\left(\bar{a}_{1} \ldots \bar{a}_{n}\right)$ for any $\bar{a}_{i}$ 's from $\operatorname{ccl}(P)$. We proceed by induction on $n$. This holds when $n=1$ by the definition of $\operatorname{ccl}(P)$. Set $\bar{x}:=\bar{a}_{1} \ldots \bar{a}_{n-1}$. By induction hypothesis and Lemma $5.12\left\{\bar{a}_{1}, \ldots, \bar{a}_{n-1}\right\}$ is coherent and $\boldsymbol{\delta}\left(\bar{x} / \bar{a}_{n}\right) \leq \mathrm{d}^{0}\left(\bar{x} / \bar{a}_{n}\right)$. So by additivity

$$
\boldsymbol{\delta}\left(\overline{x a}_{n}\right)=\boldsymbol{\delta}\left(\bar{x} / \bar{a}_{n}\right)+\boldsymbol{\delta}\left(\bar{a}_{n}\right) \leq \mathrm{d}^{0}\left(\bar{x} / \bar{a}_{n}\right)+\mathrm{d}^{0}\left(\bar{a}_{n}\right)=\mathrm{d}^{0}\left(\overline{x a}_{n}\right) .
$$

But Lemma 5.13 implies that $\boldsymbol{\delta}\left(\overline{x a}_{n}\right) \geq \mathrm{d}^{0}\left(\overline{x a}_{n}\right)$. This ends the proof.

Clearly $\operatorname{ccl}(\operatorname{ccl}(P))=\operatorname{ccl}(P)$. We say $P \subset K^{<\infty}$ is coherently algebraically closed if $P$ is coherent and $P=\operatorname{ccl}(P)$. 
Proposition 5.17. Suppose $P \subset K^{<\infty}$ is coherently algebraically closed. Then the pregeometry $\left(P ; \operatorname{acl}^{0} \uparrow_{P}\right)$ is modular.

Proof. We must show that if $B \subset P$ and $\bar{a}_{1}, \bar{a}_{2} \in P \backslash \operatorname{acl}^{0}(B)^{<\infty}$ are such that $\bar{a}_{1} \in$ $\operatorname{acl}^{0}\left(B \cup\left\{\bar{a}_{2}\right\}\right)^{<\infty}$, then $\bar{a}_{1} \in \operatorname{acl}^{0}\left(\left\{\bar{d}, \bar{a}_{2}\right\}\right)^{<\infty}$ for some $\bar{d} \in P \cap \operatorname{acl}^{0}(B)^{<\infty}$. We may assume without loss of generality that $B$ is finite, say $B=\left\{\bar{a}_{3}, \ldots, \bar{a}_{n}\right\}$. This is the situation of Proposition 5.14 from which we conclude that there is an integer $k$ such that $k=\mathrm{d}^{0}\left(\bar{a}_{1} \bar{a}_{2} / \bar{d}\right)=\mathrm{d}^{0}\left(\bar{a}_{2}\right)=\mathrm{d}^{0}\left(\bar{a}_{1}\right)=\mathrm{d}^{0}(\bar{d})$ for some $\bar{d} \in \operatorname{acl}^{0}\left(\left\{\bar{a}_{1}, \bar{a}_{2}\right\}\right)<\infty$.

We are left to show that $\bar{d} \in P$, and since we already know that $\boldsymbol{\delta}(\bar{d})=\mathrm{d}^{0}(\bar{d})$ and $\bar{d} \in \operatorname{acl}^{0}(P)$, we are only left to check that $\bar{d}$ is cgp.

To this end assume that $\bar{d}$ is not $\operatorname{acl}^{0}$ independent from $E$, for some $E \subset K^{<\infty}$. We need to show that $\boldsymbol{\delta}(\bar{d} / E)=0$. By Fact 2.4 we may pick $\bar{a}_{1}^{\prime}, \bar{a}_{2}^{\prime} \in K^{<\infty}$ such that $\operatorname{tp}\left(\bar{a}_{1}^{\prime}, \bar{a}_{2}^{\prime} / \bar{d}\right)=\operatorname{tp}\left(\bar{a}_{1}, \bar{a}_{2} / \bar{d}\right)$ and $\boldsymbol{\delta}\left(\bar{a}_{1}^{\prime}, \bar{a}_{2}^{\prime} / E \bar{d}\right)=\boldsymbol{\delta}\left(\bar{a}_{1}^{\prime}, \bar{a}_{2}^{\prime} / \bar{d}\right)$. For brevity write $\bar{a}:=\bar{a}_{1} \bar{a}_{2}$ and $\bar{a}^{\prime}=\bar{a}_{1}^{\prime} \bar{a}_{2}^{\prime}$. By additivity of $\boldsymbol{\delta}$ we may write:

$$
\boldsymbol{\delta}(\bar{d} / E)=\boldsymbol{\delta}\left(\bar{d} / \bar{a}^{\prime} E\right)+\boldsymbol{\delta}\left(\bar{a}^{\prime} / E\right)-\boldsymbol{\delta}\left(\bar{a}^{\prime} / \bar{d} E\right) .
$$

Let us examine the three terms on the right hand side.

The first term $\delta\left(\bar{d} / \bar{a}^{\prime} E\right)$ is zero since $\bar{d} \in \operatorname{acl}^{0}\left(\bar{a}^{\prime}\right)^{<\infty}$, because $\bar{d} \in \operatorname{acl}^{0}(\bar{a})^{<\infty}$.

The second term is equal to $\boldsymbol{\delta}\left(\bar{a}_{1}^{\prime} / \bar{a}_{2}^{\prime} E\right)+\boldsymbol{\delta}\left(\bar{a}_{2}^{\prime} / E\right)$. We claim that this is at most $k$. Note that $\bar{a}_{1}^{\prime}, \bar{a}_{2}^{\prime}$ are cgp because $\bar{a}_{1}^{\prime}, \bar{a}_{2}^{\prime}$ are cgp (see Remark 3.7). By (11) it is enough to show that one of these terms is zero. Hence by the cgp we only need to check that either $\bar{a}_{1}^{\prime} \chi^{0} \bar{a}_{2}^{\prime} E$ or $\bar{a}_{2}^{\prime} \mathfrak{\ell}^{0} E$. In other words that $\bar{a}_{1}^{\prime} \bar{a}_{2}^{\prime} \mathfrak{L}^{0} E$. This is indeed the case for otherwise $\bar{d}$ would be independent from $E$, because $\bar{d} \in \operatorname{acl}^{0}\left(\bar{a}^{\prime}\right)<\infty$.

Finally let us turn to the third term. Since $\bar{d} \in \operatorname{acl}^{0}(B)^{<\infty}$ by Fact 7 we have

$$
\boldsymbol{\delta}\left(\bar{a}^{\prime} / \bar{d}\right)=\boldsymbol{\delta}(\bar{a} / \bar{d}) \geq \boldsymbol{\delta}\left(\bar{a} / \operatorname{acl}^{0}(B)\right)=\boldsymbol{\delta}(\bar{a} / B) .
$$

On the other hand since $\bar{a}_{1}, \bar{a}_{2}, B$ lie in $P$ and $P$ is coherent we have $\boldsymbol{\delta}(\bar{a} / B)=$ $\mathrm{d}^{0}(\bar{a} / B)$. By Proposition 5.14 this is $k$. Hence $\boldsymbol{\delta}\left(\bar{a}^{\prime} / \bar{d} E\right)=\boldsymbol{\delta}\left(\bar{a}^{\prime} / \bar{d}\right) \geq k$. This concludes the proof.

Proof of Theorem 5.9. By Proposition $5.10\left(P ; \mathrm{acl}^{0} \uparrow_{P}\right)$ is a pregeometry. By Lemma 5.16 , enlarging $P$ to $\operatorname{ccl}(P)$ if necessary, we may assume that $P$ is coherently algebraically closed. By Proposition 5.17 the associated geometry $\mathcal{G}_{P}$ is modular. Hence the non-orthogonality relation is an equivalence relation.

Up to enlarging $P$ further if necessary, we can assume that each non-orthogonality class in $\mathcal{G}_{P}$ of dimension $>1$ has infinite dimension. This follows from the Lemma 5.18 below applied iteratively countably many times. In each finite dimensional non-orthogonality class we pick a point $a$ and increase its dimension without altering the other classes, until all classes are infinite dimensional. Now we conclude by the Veblen-Young Theorem as recalled in Fact 5.5.

Lemma 5.18 (dimension increase). If $P$ is coherently algebraically closed and $a, b, c \in$ $P$ are distinct in $\mathcal{G}_{P}$ and collinear in the sense that $c \in \operatorname{acl}^{0}(a, b)$, then there is $a^{\prime} \in K^{<\infty}$ non-orthogonal to a such that $P^{\prime}:=\operatorname{ccl}\left(P \cup\left\{a^{\prime}\right\}\right)$ is coherent, $a^{\prime} \bigsqcup^{0} P$, and every $x \in P^{\prime}$ is either in $P$ or non-orthogonal to $a$.

Proof. Note first if $a, b \in P$ are non-orthogonal, then $\mathrm{d}^{0}(a)=\mathrm{d}^{0}(b)$. This is part of the conclusion of Proposition 5.14, or also follows easily from Lemma 5.11.

Now by Fact 2.4 we can pick $a^{\prime}, c^{\prime} \in K^{<\infty}$ with $\operatorname{tp}\left(a^{\prime} c^{\prime} / b\right)=\operatorname{tp}(a c / b)$ and $\boldsymbol{\delta}\left(a^{\prime} c^{\prime} / P\right)=\boldsymbol{\delta}(a c / b)$. Since $P$ is coherent $\boldsymbol{\delta}(a / b)=\mathrm{d}^{0}(a / b)=\mathrm{d}^{0}(a)=\boldsymbol{\delta}(a)$, while since $a, a^{\prime}$ have the same type $\boldsymbol{\delta}(a)=\boldsymbol{\delta}\left(a^{\prime}\right)$ coincides with $\mathrm{d}^{0}\left(a^{\prime}\right)=\mathrm{d}^{0}(a)$. Also $a^{\prime}$ and $c^{\prime}$ are $c g p$ (see Remark 3.7). Since $\operatorname{tp}\left(a^{\prime} c^{\prime} / b\right)=\operatorname{tp}(a c / b)$ we have $c^{\prime} \in \operatorname{acl}^{0}\left(P \cup\left\{a^{\prime}\right\}\right)$ and hence $\boldsymbol{\delta}\left(c^{\prime} / P a^{\prime}\right)=0$. Similarly, $\boldsymbol{\delta}(c / a b)=0$. By additivity 
we conclude $\boldsymbol{\delta}\left(a^{\prime} / P\right)=\boldsymbol{\delta}\left(a^{\prime} c^{\prime} / P\right)=\boldsymbol{\delta}(a c / b)=\boldsymbol{\delta}(a / b)=\mathrm{d}^{0}(a)$. Hence also $a^{\prime} \bigsqcup^{0} P$ since $a^{\prime}$ is cgp. We conclude $\mathrm{d}^{0}\left(a^{\prime} / P\right)=\boldsymbol{\delta}\left(a^{\prime} / P\right)$. It follows from Lemma 5.12 and additivity that $P \cup\left\{a^{\prime}\right\}$ is also coherent.

By Lemma 5.16 $P^{\prime}=\operatorname{ccl}\left(P \cup\left\{a^{\prime}\right\}\right)$ is also coherent. Moreover $b, a^{\prime}, c^{\prime}$ are collinear so $a^{\prime}$ is non-orthogonal to $a, b$ and $c$. Finally if $x \in P^{\prime}$, then $x \in \operatorname{acl}^{0}\left(P \cup\left\{a^{\prime}\right\}\right)$, so if $x \notin P \cup \operatorname{acl}^{0}\left(a^{\prime}\right)$ by modularity there is $y \in P$ such that $x, a^{\prime}, y$ are collinear, hence $a^{\prime}$ and $x$ are non-orthogonal.

Remark 5.19. The results of this section go through with the same proofs when $A C F_{0}$ is replaced by an arbitrary finite U-rank theory in which Lemma 2.15 holds.

\section{VARIETIES WITH COHERENT GENERICS}

We now show in Proposition 6.1 below that the locus of a coherent tuple is a special variety. This will follow from Theorem 5.9 and a characterisation of the projective geometries which can arise from $\mathrm{acl}^{0}$. We shall give such a characterisation in Appendix A, generalising a result of Evans-Hrushovski.

Proposition 6.1. Suppose $a_{1}, \ldots, a_{n} \in K^{<\infty}$ are such that $\bar{a}=\left(a_{1}, \ldots, a_{n}\right)$ is coherent. Then $\operatorname{loc}^{0}(\bar{a})$ is a special subvariety of $\prod_{i} \operatorname{loc}^{0}\left(a_{i}\right)$.

Remark 6.2. Technically, we defined "special" only for complex varieties, but $\operatorname{loc}^{0}(\bar{a})$ is a variety over $C_{0}$ and $C_{0}$ need not come with an embedding into $\mathbb{C}$. In our main applications in Section 7 below, $C_{0}$ will come with such an embedding; more generally, we may take an arbitrary such embedding, or just define "special" for varieties over an algebraically closed field $C_{0}$ by exact analogy to the definition for varieties over $\mathbb{C}$ in the introduction.

Proof. In this proof we make use of some of the definitions from Appendix A, applied to the pair of algebraically closed fields $C_{0} \leq K$. In particular, given $x \in K^{<\infty} \backslash \operatorname{acl}^{0}(\emptyset)$ we set $\widetilde{x}:=\operatorname{acl}^{0}(x)$, and we let $\mathcal{G}_{K}$ be the projectivisation of the closure structure $\left(K^{<\infty}, \mathrm{acl}^{0}\right)$ defined in Example 5.3 above, namely $\mathcal{G}_{K}:=$ $\mathbb{P}\left(K^{<\infty} ; \operatorname{acl}^{0}\right)=\left\{\widetilde{x}: x \in K^{<\infty} \backslash \operatorname{acl}^{0}(\emptyset)\right\}$.

We may assume no $a_{i}$ lies in $\operatorname{acl}^{0}(\emptyset)$. Indeed, if say $a_{1} \in \operatorname{acl}^{0}(\emptyset)$, then $\operatorname{loc}^{0}(\bar{a})=$ $\left\{a_{1}\right\} \times \operatorname{loc}^{0}\left(a_{2}, \ldots, a_{n}\right)$, and $\left\{a_{1}\right\}$ is special (with the trivial group, which is a special subgroup of itself), and so it suffices to show that $\operatorname{loc}^{0}\left(a_{2}, \ldots, a_{n}\right)$ is special.

By Theorem 5.9, $\left\{a_{1}, \ldots, a_{n}\right\}$ extends to a coherent set $P$ such that $\mathcal{G}_{P}=\{\widetilde{p}$ : $p \in P\} \subset \mathcal{G}_{K}$ splits as a sum of 1-dimensional and infinite dimensional projective geometries over division rings. This induces a corresponding splitting of $\bar{a}$ into subtuples of $a_{i}$ 's, and the locus of $\bar{a}$ is the product of their loci. So it suffices to show that each such locus is special. So we may assume $\widetilde{a}_{1}, \ldots, \widetilde{a}_{n}$ are all contained in a single summand.

We conclude by showing that $\operatorname{loc}^{0}(\bar{a})$ is in co-ordinatewise correspondence with a special subgroup, by finding a commutative algebraic group $G$ over $C_{0}$ and generics $h_{i} \in G(K)$ with $\widetilde{a}_{i}=\widetilde{h}_{i}$, such that $\operatorname{loc}^{0}(\bar{h})$ is a special subgroup of $G^{n}$. By Remark 2.11, this will suffice.

If $\widetilde{a_{i}}=\widetilde{a_{j}}$ for $i \neq j$, we can take $h_{j}:=h_{i}$. So assume there are no such interalgebraicities. Let $\mathcal{G}_{\bar{a}}:=\left\{\widetilde{a}_{1}, \ldots, \widetilde{a}_{n}\right\} \subset \mathcal{G}_{P} \subset \mathcal{G}_{K}$.

If $\operatorname{dim}\left(\mathcal{G}_{\bar{a}}\right)=1$ (the "trivial" case), then $\bar{a}=a_{1}$ and we may take $G:=\mathbb{G}_{a}^{\mathrm{d}^{0}\left(a_{1}\right)}$ and a point $h_{1} \in G(K)=K^{\mathrm{d}^{0}\left(a_{1}\right)}$ with $\widetilde{a_{1}}=\widetilde{h_{1}}$.

Else, $\mathcal{G}_{\bar{a}}$ embeds in a projective geometry over a division ring, where moreover by Lemma 5.11 the latter geometry is fully embedded in $\mathcal{G}_{K}$ in the sense of Definition A.2. So by Proposition A.4, there is an abelian algebraic group $G$ over $C_{0}$ with 
$\operatorname{dim}(G)=\mathrm{d}^{0}\left(a_{i}\right)$, and a division subring $F$ of $\operatorname{End}_{C_{0}}^{0}(G)$, and $\bar{h}=\left(h_{1}, \ldots, h_{n}\right) \in$ $G(K)^{n}$ with $\widetilde{h_{i}}=\widetilde{a_{i}}$, such that (in particular) $\operatorname{dim}_{F}\left(\left\langle\bar{h} / G\left(C_{0}\right)\right\rangle_{F}\right)=\operatorname{dim}\left(\mathcal{G}_{\bar{a}}\right)$.

Hence $A \cdot\left(\bar{h} / G\left(C_{0}\right)\right)=0$ for some $A \in \operatorname{Mat}_{n}(F)$ of rank $n-\operatorname{dim}\left(\mathcal{G}_{\bar{a}}\right)$. By clearing denominators, we may assume that $A$ has entries from $\operatorname{End}_{C_{0}}(G) \cap F$.

Let $\bar{c}:=A \cdot \bar{h} \in G\left(C_{0}\right)^{n}$. Since $C_{0}$ is algebraically closed, $A \cdot \bar{x}=\bar{c}$ has a solution $\bar{h}_{0} \in G\left(C_{0}\right)^{n}$. Replacing $\bar{h}$ by $\bar{h}-\bar{h}_{0}$, which does not affect $\widetilde{h}_{i}$, we may assume $\bar{h} \in \operatorname{ker}(A)$. Write $\operatorname{ker}(A)^{o}$ for the connected component of $\operatorname{ker}(A)$. By further replacing $\bar{h}$ by $e \cdot \bar{h}$ where $e \in \mathbb{N}$ is the exponent of the finite group $\operatorname{ker}(A) / \operatorname{ker}(A)^{\circ}$, we may assume $\bar{h} \in \operatorname{ker}(A)^{\circ}$. Now it is not hard to see, e.g. by considering Gaussian elimination, that $\operatorname{dim}(\operatorname{ker}(A))=\operatorname{dim}(G)(n-\operatorname{rank}(A))$. So $\operatorname{dim}\left(\operatorname{ker}(A)^{\circ}\right)=$ $\operatorname{dim}(G)(n-\operatorname{rank}(A))=\mathrm{d}^{0}(\bar{a})=\mathrm{d}^{0}(\bar{h})$. Therefore $\operatorname{loc}^{0}(\bar{h})=\operatorname{ker}(A)^{\circ}$ is a special subgroup of $G^{n}$ as required.

\section{Asymptotic consequences}

In this section we first unpeel the ultraproduct construction to show how coherent tuples correspond to varieties without powersaving. Then, combining this with Proposition 6.1 above and some further argument, we prove the combinatorial theorems stated in the introduction.

Let $W_{i}, i=1, \ldots, n$, be irreducible complex algebraic varieties each of dimension $d$, and let $V \subset \prod_{i} W_{i}$ be an irreducible complex closed subvariety. We first recall the following simple observation, already mentioned in the introduction.

Lemma 7.1 (the trivial bound). Let $\tau>d$. There is $C \in \mathbb{N}$ depending only on $\tau$ and $V$ such that if $X_{i} \subset W_{i}$ is in $(C, \tau)$-coarse general position in $W_{i}$ (see Def. 1.7) and if $\left|X_{i}\right| \leq N^{d}$, then $\left|V \cap \prod_{i \leq n} X_{i}\right| \leq O_{V}\left(N^{\operatorname{dim}(V)}\right)$. Furthermore, if $V$ admits no power-saving then $\operatorname{dim}(V)$ is an integral multiple of $d$.

Proof. We prove this by induction on $n$ and $\operatorname{dim}(V)$, with $C$ and the multiplicative constant in $O_{V}$ depending only on the complexity of $V$ and the $W_{i}$ 's. For $n=1$ it is clear. For $n>1$, consider the projection $\pi: V \rightarrow \prod_{i<n} W_{i}$. Let $Y$ be the Zariski closure of the image $\pi(V)$. Then $Y$ is irreducible since $V$ is. By [BGT11, Lemma 3.7], there is a proper closed subvariety $Z \subsetneq Y$ such that for $y \in \pi(V) \backslash Z$ the fibre $\pi^{-1}(y)$ has dimension $D:=\operatorname{dim}(V)-\operatorname{dim}(Y)$ and both $Y$ and $Z$ as well as the fibers $\pi^{-1}(y)$ have complexity bounded by a constant depending only on the complexity of $V$ and the $W_{i}$ 's. We may assume that $\tau$ is larger than this constant.

Now $V^{\prime}:=\pi^{-1}(Z)$ is a proper closed subvariety of $V$, so by the inductive hypothesis applied to its irreducible components, $\left|V^{\prime} \cap \prod_{i \leq n} X_{i}\right| \leq O_{V}\left(N^{\operatorname{dim}(V)-1}\right)$.

If $D=0$, the fibres over $\pi(V) \backslash Z$ have size uniformly bounded by some $c \in \mathbb{N}$, and so

$$
\left|\left(V \backslash V^{\prime}\right) \cap \prod_{i \leq n} X_{i}\right| \leq c\left|Y \cap \prod_{i<n} X_{i}\right|
$$

and we conclude by the inductive hypothesis and $\operatorname{dim}(V)=\operatorname{dim}(Y)$.

If $D=d$, we conclude by the trivial estimate

$$
\left|\left(V \backslash V^{\prime}\right) \cap \prod_{i \leq n} X_{i}\right| \leq N^{d}\left|Y \cap \prod_{i<n} X_{i}\right|
$$

and $\operatorname{dim}(V)=d+\operatorname{dim}(Y)$.

If $0<D<d$, by $\tau$-coarse general position of $X_{n}$, and the inductive hypothesis

$$
\left|\left(V \backslash V^{\prime}\right) \cap \prod_{i \leq n} X_{i}\right| \leq O\left(N^{\frac{d}{\tau}}\left|Y \cap \prod_{i<n} X_{i}\right|\right) \leq O\left(N^{\frac{d}{\tau}+\operatorname{dim}(V)-D}\right) ;
$$

so we see that for $\tau>d$ the desired bound holds, and moreover that $V$ admits a power-saving. Finally if the projection of $V$ to $W_{i}$ for some $i$ is not dominant, then 
has no power saving, then so does $Y$ and $V$ has dominant projections on all $W_{i}$ 's with $i<n$.

Let $C_{0} \subset \mathbb{C}$ be a countable algebraically closed field over which $V$ and the $W_{i}$ 's are defined. Consider as in $\S 2.1$ a sequence $K_{s}$ of $\mathcal{L}$-structures on the complex field $\mathbb{C}$ and a scaling constant $\xi \in{ }^{*} \mathbb{R}$ so as to form the coarse pseudo-finite dimension $\boldsymbol{\delta}$ defined on subsets of tuples with co-ordinates in $K:=\prod_{s \rightarrow \mathcal{U}} K_{s}$ for some nonprincipal ultrafilter $\mathcal{U}$. Here as before $\mathcal{L}$ is a countable language expanding the language of rings on $(K,+, \cdot)$ and containing a constant symbol for each $c \in C_{0}$, and closed under cardinality quantifiers so as to make $\boldsymbol{\delta}$ invariant and continuous (cf. 2.1 .6$)$.

For an irreducible algebraic variety $W$ defined over $C_{0}$, we will say that an internal set $X \subset W(K)$ is $\boldsymbol{c g p}$ in $W$ if $0<\boldsymbol{\delta}(X)<\infty$ and for any proper closed subvariety $W^{\prime} \subsetneq W$ over $K$, we have $\delta\left(X \cap W^{\prime}(K)\right)=0$.

Lemma 7.2. Suppose $X \subset W(K)$ is an internal set which is cgp in $W$. Then any $a \in X$ is cgp.

Proof. Suppose $B \subset K^{<\infty}$ and $a \mathfrak{L}^{0} B$. Then $W^{\prime}:=\operatorname{locus}^{W}(a / B)$ is a proper subvariety of $W$, and so $\delta(a / B) \leq \boldsymbol{\delta}\left(X \cap W^{\prime}\right)=0$.

We also introduce one last piece of terminology:

Definition 7.3. We will say that an element $a \in W(K)$ is $\boldsymbol{d} \boldsymbol{c g} \boldsymbol{p} \boldsymbol{i n} W$ (for definably in coarse general position) if it is contained in a subset $X \subset W(K)$, which is definable without parameters in $\mathcal{L}$ and is cgp in $W$.

It is immediate from Lemma 7.2 that every dcgp tuple $\bar{a}$ is cgp. Recall that $W_{1}, \ldots, W_{n}$ are irreducible complex algebraic varieties of dimension $d$.

Lemma 7.4. Let $V \subset \prod_{i} W_{i}$ be an irreducible complex closed subvariety. The following are equivalent:

(1) The subvariety $V$ admits no power-saving,

(2) (existence of a coherent generic) for some language $\mathcal{L}$ as above there are some $\bar{a}_{i} \in W_{i}(K)$ such that $\bar{a}=\left(\bar{a}_{1}, \ldots, \bar{a}_{n}\right) \in V(K)$ is coherent and generic in $V$ with $\bar{a}_{i}$ degp in $W_{i}$ for each $i$.

Proof. If $V$ admits no power-saving, then for any $s \in \mathbb{N}$, setting $\tau:=1+s$ and $\epsilon:=\frac{1}{\tau}$, there exists $N_{s} \geq s$ and $\left|X_{i, s}\right| \leq N_{s}^{d}$ such that $\left|V \cap \prod_{i} X_{i, s}\right|>N_{s}^{\operatorname{dim} V-\epsilon}$ and $\left|X_{i, s}^{\tau} \cap W_{i}^{\prime}\right| \leq\left|X_{i, s}\right|^{\epsilon}$ for any $W_{i}^{\prime}$ a proper closed subvariety of $W_{i}$ of complexity $\leq \tau$.

After enlarging $\mathcal{L}$ if necessary, we may assume that $\prod_{s \rightarrow \mathcal{U}} X_{i, s}=: X_{i}$ are definable without parameters in $\mathcal{L}$. Set the scaling constant $\xi:=\lim _{s \rightarrow \mathcal{U}} N_{s}$. Then by the above estimates and Lemma $7.1, \boldsymbol{\delta}\left(V \cap \prod_{i} X_{i}\right)=\operatorname{dim} V$. So by Fact 2.4, say $\bar{a}=\left(\bar{a}_{1}, \ldots, \bar{a}_{n}\right) \in V \cap \prod_{i=1}^{n} X_{i}$ with $\boldsymbol{\delta}(\bar{a})=\operatorname{dim} V$. By construction each $X_{i}$ is cgp in $W_{i}$, so $\bar{a}_{i}$ is dcgp and hence cgp (Lemma 7.2). In particular $\delta\left(\bar{a}_{i}\right) \leq \mathrm{d}^{0}\left(\bar{a}_{i}\right)$, for $\boldsymbol{\delta}\left(\bar{a}_{i}\right) \leq d$ since $\bar{a}_{i} \in X_{i}$ and either $\mathrm{d}^{0}\left(\bar{a}_{i}\right)=d$ or $\bar{a}_{i}$ is contained in a proper subvariety of $W_{i}$ defined over $C_{0}$, which forces $\boldsymbol{\delta}\left(\bar{a}_{i}\right)=0$, since $X_{i}$ is cgp in $W_{i}$. Also $\bar{a}$ is generic in $V$, i.e. $\mathrm{d}^{0}(\bar{a})=\operatorname{dim} V$, for otherwise $\bar{a} \in V^{\prime}$ for some proper irreducible subvariety $V^{\prime}$ of $V$ defined over $C_{0}$ and hence by the trivial bound of Lemma 7.1 we would have $\boldsymbol{\delta}(\bar{a}) \leq \boldsymbol{\delta}\left(V^{\prime} \cap \prod_{i=1}^{n} X_{i}\right) \leq \operatorname{dim} V-1$. It then follows from Lemma 5.12 that $\bar{a}$ is coherent.

Suppose conversely that, for some $K_{s}$ and $\xi \in{ }^{*} \mathbb{R}$, we have a tuple $\bar{a} \in V(K)$ which is coherent generic and for each $i$ we have $\bar{a}_{i} \in X_{i}$, an $\mathcal{L}$-definable without parameters and cgp subset of $W_{i}(K)$. To say that $\bar{a}$ is coherent means that $\left\{\bar{a}_{1}, \ldots, \bar{a}_{n}\right\}$ is a coherent set. In particular $\bar{a}_{i}$ is cgp and $\boldsymbol{\delta}\left(\bar{a}_{i}\right)=\mathrm{d}^{0}\left(\bar{a}_{i}\right)$. Since $\bar{a}$ is 
generic in $V$, its projection $\bar{a}_{i}$ is generic in the co-ordinate projection $\pi_{i}(V) \subset W_{i}$. We may assume that this projection is dominant, for otherwise by cgp we would have $\boldsymbol{\delta}\left(\bar{a}_{i}\right)=0$ and hence $\mathrm{d}^{0}\left(\bar{a}_{i}\right)=0$, which would mean that the projection $\pi_{i}(V)$ is a point, and we may replace $V$ with the fibre of this projection and omit $W_{i}$. So we have $\boldsymbol{\delta}\left(\bar{a}_{i}\right)=\mathrm{d}^{0}\left(\bar{a}_{i}\right)=d$.

Now let $\epsilon>0$ and $\tau \in \mathbb{N}$. Pick $Y_{i} \subset W_{i}(K)$ definable over $\emptyset$ with $\bar{a}_{i} \in Y_{i}$ and $d \leq \boldsymbol{\delta}\left(Y_{i}\right)<d+\epsilon$. Replacing $Y_{i}$ by $Y_{i} \cap X_{i}$ we may assume $Y_{i}$ is cgp in $W_{i}$. Then $\boldsymbol{\delta}\left(\bar{V} \cap \prod_{i} Y_{i}\right) \geq \boldsymbol{\delta}(\bar{a})=\operatorname{dim} V$. Let $Y_{i, s}:=Y_{i}^{K_{s}}$ be the interpretation in $K_{s}$. Then for $\mathcal{U}$-many $s$, for all $i \in\{1, \ldots, n\}, Y_{i, s}$ is $\tau$-cgp in $W_{i}, 1 / \epsilon<\left|Y_{i, s}\right|<\infty$, and $\left|V \cap \prod_{i} Y_{i, s}\right| \geq\left|Y_{i, s}\right|^{\frac{\operatorname{dim} V}{d+2 \epsilon}}$. Hence $V$ admits no power-saving.

7.1. Sharpness. In this subsection, we show the converse to Proposition 6.1 and prove that every special subvariety has no power saving. For this we will need to construct certain well chosen cartesian products of finite sets, which are adapted to the special subvariety.

The construction we are about to describe consists in building certain long multidimensional arithmetic progressions on few algebraically independent elements. The difficulty is that in order to belong to a given special subgroup, these progressions will need to satisfy some almost invariance under the division $\operatorname{ring} F$ of $E n d^{0}(G)$ used to define the special subgroup. For this purpose it will be convenient to introduce the notion of constrainedly filtered ring, as follows.

Definition 7.5. A constrained filtration of a ring $\mathcal{O}$ is a chain $\mathcal{O}_{n} \subset \mathcal{O}$ of finite subsets, $n \in \mathbb{N}$, such that

(CF0) $\bigcup_{n \in \mathbb{N}} \mathcal{O}_{n}=\mathcal{O}$, and $\forall n \in \mathbb{N}$. $\mathcal{O}_{n} \subset \mathcal{O}_{n+1}$;

(CF1) $\exists k \in \mathbb{N} . \forall n \in \mathbb{N}$. $\mathcal{O}_{n}+\mathcal{O}_{n} \subset \mathcal{O}_{n+k}$;

(CF2) $\forall a \in \mathcal{O} . \exists k \in \mathbb{N} . \forall n \in \mathbb{N} . a \mathcal{O}_{n} \subset \mathcal{O}_{n+k}$;

(CF3) $\forall \epsilon>0$. $\frac{\left|\mathcal{O}_{n+1}\right|}{\left|\mathcal{O}_{n}\right|} \leq O_{\epsilon}\left(\left|\mathcal{O}_{n}\right|^{\epsilon}\right)$.

If a constrained filtration exists, we say $\mathcal{O}$ is constrainedly filtered.

Example 7.6. $\mathbb{Z}$ is constrainedly filtered, since $\left(\left[-2^{n}, 2^{n}\right]\right)_{n}$ is a constrained filtration.

Constrained filtrations are somewhat similar to Bourgain systems.

Lemma 7.7. Suppose $\mathcal{O}$ is a constrainedly filtered ring.

(i) The polynomial ring $\mathcal{O}[X]$ is constrainedly filtered.

(ii) If $\mathcal{O}$ is an integral domain and $a \in \mathcal{O}$, then the subring $\mathcal{O}\left[a^{-1}\right]$ of the fraction field of $\mathcal{O}$ is constrainedly filtered.

(iii) If $\mathcal{O}^{\prime} \supset \mathcal{O}$ is an extension ring in which $\mathcal{O}$ is central and which is free and finitely generated as a $\mathcal{O}$-module, then $\mathcal{O}^{\prime}$ is constrainedly filtered.

Proof. Say $\left(\mathcal{O}_{n}\right)_{n}$ is a constrained filtration of $\mathcal{O}$.

(i) Let $\mathcal{O}_{n}^{\prime}:=\sum_{i<n} \mathcal{O}_{n} X^{i}$. Then (CF0)-(CF2) are easily verified. For (CF3), note that $\left|\mathcal{O}_{n}^{\prime}\right|=\left|\mathcal{O}_{n}\right|^{n}$, and so for $\epsilon>0$, for $n>>0,\left|\mathcal{O}_{n+1}^{\prime}\right| /\left|\mathcal{O}_{n}^{\prime}\right|=$ $\left|\mathcal{O}_{n}\right|\left(\left|\mathcal{O}_{n+1}\right| /\left|\mathcal{O}_{n}\right|\right)^{n+1} \leq O_{\epsilon}\left(\left|\mathcal{O}_{n}\right|^{1+(1+n) \epsilon}\right) \leq O_{\epsilon}\left(\left|\mathcal{O}_{n}^{\prime}\right|^{\frac{1}{n}+\frac{1+n}{n} \epsilon}\right) \leq O_{\epsilon}\left(\left|\mathcal{O}_{n}^{\prime}\right|^{2 \epsilon}\right)$.

(ii) Say $k \in \mathbb{N}$ is such that for all $n \in \mathbb{N}$ we have $a \mathcal{O}_{n} \subset \mathcal{O}_{n+k}$ and $\mathcal{O}_{n}+$ $\mathcal{O}_{n} \subset \mathcal{O}_{n+k}$. Let $\mathcal{O}_{n}^{\prime}:=a^{-n} \mathcal{O}_{2 k n}$. Then $\mathcal{O}_{n}^{\prime}+\mathcal{O}_{n}^{\prime}=a^{-n}\left(\mathcal{O}_{2 k n}+\mathcal{O}_{2 k n}\right) \subset$ $a^{-n}\left(\mathcal{O}_{2 k n+k}\right)=a^{-(n+1)}\left(a \mathcal{O}_{2 k n+k}\right) \subset a^{-(n+1)}\left(\mathcal{O}_{2 k n+k+k}\right)=\mathcal{O}_{n+1}^{\prime}$, so (CF1) holds.

$(\mathrm{CF} 0)$ and $(\mathrm{CF} 2)$ are immediate, and (CF3) holds since $\left|\mathcal{O}_{n}^{\prime}\right|=\left|\mathcal{O}_{2 k n}\right|$.

(iii) Say $\mathcal{O}^{\prime}=\bigoplus_{i=1}^{d} a_{i} \mathcal{O}$. Then let $\mathcal{O}_{n}^{\prime}:=\bigoplus_{i=1}^{d} a_{i} \mathcal{O}_{n}$. 
Then (CF0), (CF1), and (CF3) are clear. For (CF2), let $c_{i j}^{t} \in \mathcal{O}$ be such that $a_{i} a_{j}=\sum_{t} c_{i j}^{t} a_{t}$; then given $\beta=\sum a_{i} b_{i} \in \mathcal{O}^{\prime}$ with $b_{i} \in \mathcal{O}$, let $k:=\max _{i, j, t}\left(k_{b_{i}}+k_{c_{i j}^{t}}\right)$ where $\alpha \mathcal{O}_{n} \subset \mathcal{O}_{n+k_{\alpha}}(\forall n)$, and say $\mathcal{O}_{n}+\mathcal{O}_{n} \subset$ $\mathcal{O}_{n+l}(\forall n)$. Then $\beta \mathcal{O}_{n}^{\prime}=\sum_{j} \beta a_{j} \mathcal{O}_{n}=\sum_{i, j} a_{i} b_{i} a_{j} \mathcal{O}_{n}=\sum_{i, j} b_{i} a_{i} a_{j} \mathcal{O}_{n}=$ $\sum_{i, j, t} b_{i} c_{i j}^{t} a_{t} \mathcal{O}_{n} \subset \sum_{i, j, t} a_{t} \mathcal{O}_{n+k} \subset \mathcal{O}_{n+k+d^{2} l}^{\prime}$

Lemma 7.8. Suppose $D$ is a finite-dimensional algebra over a characteristic 0 field $L$, and $\mathcal{O} \subset D$ is a finitely generated subring. Then there exists a constrainedly filtered subring $\mathcal{O}^{\prime} \subset D$ extending $\mathcal{O}$.

Proof. Let $\left(e_{k}\right)_{1 \leq k \leq d}$ be an $L$-basis of $D$ and $\left(f_{j}\right)_{j}$ generators of $\mathcal{O}$. Without loss of generality we may change $L$ into the subfield generated by the co-ordinates $f_{j}^{k}$ of the $f_{j}$ 's and the co-ordinates $c_{i j}^{k}$ of the products $e_{i} e_{j}$ 's. So we may assume that $L$ is finitely generated. Let $\bar{z}=\left(z_{1}, \ldots, z_{n}\right)$ be a transcendence basis for $L$ over $\mathbb{Q}$. Then $[L: \mathbb{Q}(\bar{z})]$ is finite, so $D$ is again finite-dimensional over $\mathbb{Q}(\bar{z})$, and without loss of generality, we may assume that $L=\mathbb{Q}(\bar{z})$. There is a polynomial $g \in \mathbb{Z}[\bar{z}]$ such that all $f_{j}^{k}$ and $c_{i j}^{k}$ belong to $\mathbb{Z}\left[\bar{z}, \frac{1}{g}\right]$. By Example 7.6, $\mathbb{Z}$ is constrainedly filtered. Then by Lemma $7.7(\mathrm{i})$, so is $\mathbb{Z}[\bar{z}]$, and by item $(i i)$ so is $R:=\mathbb{Z}\left[\bar{z}, \frac{1}{g}\right]$, and by item (iii) so is $\mathcal{O}^{\prime}:=\sum_{k=1}^{d} R e_{k} \supset \mathcal{O}$.

Fact 7.9. A division subring of a matrix algebra over a division ring has finite dimension over its centre.

Proof. This is a special case of the Kaplansky-Amitsur theorem [Jac75, p17], which shows that any primitive algebra satisfying a proper polynomial identity is finite dimensional over its centre. Indeed, any division ring is a primitive algebra, and any matrix algebra $M_{n}(\Delta)$ over a division ring $\Delta$ satisfies a polynomial identity (e.g. by the Amitsur-Levitzki theorem [Jac75, p21]).

In particular, combining this fact with the previous lemma, we see that if $F$ is a division subring of a matrix algebra, then every finite subset of $F$ is contained in a constrainedly filtered subring of $F$. We will use this observation in the next proposition. Although this is sufficient for our purposes, we do not know it to be the optimal result along these lines - in fact, for all we know, it could be that every finitely generated subring of $M_{n}(\mathbb{C})$ is constrainedly filtered.

Proposition 7.10. Suppose $V \subset \prod_{i} W_{i}$ is special. Then, for appropriate choices of $C_{0} \leq \mathbb{C}$ and structures $K_{s}$ with universe $\mathbb{C}$ and scaling constant $\xi \in{ }^{*} \mathbb{R}$, the variety $V$ has a coherent generic $\bar{a} \in V(K)$ such that each $a_{i}$ is dcgp in $W_{i}$.

Proof. The conclusion is preserved by taking products and under correspondences, so we may assume $W_{i}=G$ where $G$ is a $d$-dimensional commutative connected algebraic group defined over a countable algebraically closed subfield $C_{0} \subset \mathbb{C}$, and $V=H \leq G^{n}$ is a special subgroup. By Remark 1.9 and permuting co-ordinates, we may assume that the Lie subalgebra $\operatorname{Lie}(H) \leq \operatorname{Lie}(G)^{n}$ is the graph of an $F$ linear map $\theta=\left(\theta_{1}, \ldots, \theta_{m}\right): \operatorname{Lie}(G)^{m} \rightarrow \operatorname{Lie}(G)^{n-m}$ with $\theta_{i}(\bar{x})=\sum_{j=1}^{d} \alpha_{i j} x_{j}$, where $\alpha_{i j} \in F$ and $F$ is a division subring of $\operatorname{End}^{0}(G):=\operatorname{End}(G) \otimes_{\mathbb{Z}} \mathbb{Q}$. We may assume that $F$ is generated by the $\alpha_{i j}$. Extending $C_{0}$ if necessary, we may assume $F \subset \operatorname{End}_{C_{0}}^{0}(G)$, i.e. that every element of $F$ is a scalar multiple of an algebraic endomorphism of $G$ which is defined over $C_{0}$.

Now $F$ acts faithfully by $\mathbb{C}$-linear maps on $\operatorname{Lie}(G) \cong \mathbb{C}^{d}$, so by Fact $7.9, F$ has finite dimension over its centre. So by Lemma 7.8, there is a constrainedly filtered subring $\mathcal{O} \subset F$ such that $\alpha_{i j} \in \mathcal{O}(\forall i, j)$. 
Say $\left(\mathcal{O}_{n}\right)_{n}$ is a constrained filtration for $\mathcal{O}$. Let $\exp _{G}: \operatorname{Lie}(G) \rightarrow G(\mathbb{C})$ be the Lie exponential map, which is a surjective $\operatorname{End}(G)$-homomorphism.

For every positive integer $s$, let $\bar{\gamma}_{s}=\left(\gamma_{s, i}\right)_{i=1, \ldots, s} \in G(\mathbb{C})^{s}$ be algebraically generic over $C_{0}$, i.e. $\operatorname{dim}^{0}\left(\bar{\gamma}_{s}\right)=s d$, and let $\bar{\gamma}_{s}^{\prime} \in \operatorname{Lie}(G)^{s}$ be arbitrary such that $\exp _{G}\left(\gamma_{s, i}^{\prime}\right)=\gamma_{s, i}$. Note then that $\bar{\gamma}_{s}^{\prime}$ is $F$-linearly independent modulo $\operatorname{ker}\left(\exp _{G}\right)$.

For $k \in \mathbb{N}_{\geq 0}$ we set $X_{k, s}:=\sum_{i=1}^{s} \mathcal{O}_{s-k} \gamma_{s, i}^{\prime} \subset \operatorname{Lie}(G)$ if $s \geq k$ and $X_{k, s}:=\emptyset$ if $s<k$. Let $X_{k}:=\prod_{s \rightarrow \mathcal{U}} X_{k, s} \subset \operatorname{Lie}(G)(K):=(\operatorname{Lie}(G))^{\mathcal{U}}$. Set the scaling constant $\xi:=\left|X_{0}\right|^{\frac{1}{d}}$, so that $\delta\left(X_{0}\right)=d$.

Claim 7.11. Let $Z:=\bigcap_{k \geq 0} X_{k} \subset \operatorname{Lie}(G)(K)$. Then $Z$ is an $\mathcal{O}$-submodule with $\delta(Z)=d$.

Proof. By (CF1), there is $k_{0}$ such that for all $k$ and $s$ we have $X_{k, s}+X_{k, s} \subset X_{k-k_{0}, s}$. It follows that $Z+Z \subset Z$. Similarly, (CF2) implies $a Z \subset Z$ for all $a \in \mathcal{O}$. Finally, by (CF3), for any $k, s$ and $\epsilon \in \mathbb{R}_{>0}$, we have $\frac{\left|X_{k, s}\right|}{\left|X_{k+1, s}\right|}=\frac{\left|\mathcal{O}_{s-k}\right|^{s}}{\left|\mathcal{O}_{s-k-1}\right|^{s}} \leq O_{\epsilon}\left(\left|\mathcal{O}_{s-k-1}\right|^{s \epsilon}\right)=$ $O_{\epsilon}\left(\left|X_{k+1, s}\right|^{\epsilon}\right)$. Hence $\boldsymbol{\delta}\left(X_{k}\right) \leq(1+\epsilon) \boldsymbol{\delta}\left(X_{k+1}\right)$ for any $\epsilon>0$, so $\boldsymbol{\delta}\left(X_{k}\right) \leq \boldsymbol{\delta}\left(X_{k+1}\right)$. Clearly $\boldsymbol{\delta}\left(X_{k+1}\right) \leq \boldsymbol{\delta}\left(X_{k}\right)$, so $\boldsymbol{\delta}\left(X_{k+1}\right)=\boldsymbol{\delta}\left(X_{k}\right)$. So by induction, $\boldsymbol{\delta}\left(X_{k}\right)=d$ for all $k$, so $\boldsymbol{\delta}(Z)=\inf _{k} \boldsymbol{\delta}\left(X_{k}\right)=d$.

Now since $Z$ is an $\mathcal{O}$-submodule, the co-ordinate projection to $\operatorname{Lie}(G)^{m}$ induces a bijection from $\operatorname{Lie}(H) \cap Z^{n}$ to $Z^{m}$, so $\boldsymbol{\delta}\left(\operatorname{Lie}(H) \cap Z^{n}\right)=m d$. Moreover $\exp _{G}$ is injective on each $X_{k, s}$ and hence on each $X_{k}$ and on $Z$.

Claim 7.12. $\exp _{G}\left(X_{0}\right)$ is cgp in $G$.

Proof. Suppose $W \subsetneq G$ is a proper closed subvariety over $K$. Say $C_{0}(\bar{b}) \subset K$ is a finitely generated extension of $C_{0}$ such that $W$ is over $C_{0}(\bar{b})$. Then $W=J_{\bar{b}}$ for some $J_{\bar{x}}$ a constructible family defined over $C_{0}$ of proper closed subvarieties of $G$. If $\bar{b}=\lim _{s \rightarrow \mathcal{U}} \bar{b}_{s}$ and $W_{s}:=J_{\bar{b}_{s}}$ then $W(K)=\prod_{s \rightarrow \mathcal{U}} W_{s}(\mathbb{C})$.

It holds for $\mathcal{U}$-many $s$ that $\operatorname{dim}^{0}\left(\bar{b}_{s}\right) \leq \operatorname{dim}^{0}(\bar{b})=: k$. We claim that for such $s$, we have $\operatorname{rk}_{F}\left(\exp _{G}^{-1}\left(W_{s}(\mathbb{C})\right) \cap X_{0, s}\right) \leq k$.

Indeed, suppose $\bar{g}^{\prime}=\left(g_{i}\right)_{i=0}^{k}$ is $F$-linearly independent with $g_{i}^{\prime} \in \exp _{G}^{-1}\left(W_{s}(\mathbb{C})\right) \cap$ $X_{0, s}$. By $F$-linear algebra, some $k+1$-subtuple $\bar{\gamma}^{\prime \prime}$ of the generators $\bar{\gamma}_{s}^{\prime}$ of $X_{0, s}$ is in the $F$-linear span of $\bar{g}^{\prime}$. Let $g_{i}:=\exp _{G}\left(g_{i}^{\prime}\right)$. Then $\operatorname{dim}^{0}(\bar{g}) \geq \operatorname{dim}^{0}\left(\exp _{G}\left(\bar{\gamma}^{\prime \prime}\right)\right)=$ $(k+1) d$, so $\operatorname{dim}^{0}(\bar{g})=(k+1) d$.

Then $\operatorname{dim}^{0}\left(\bar{g} / \bar{b}_{s}\right) \leq \operatorname{dim}\left(W_{s}^{k+1}\right) \leq(k+1)(d-1)=\operatorname{dim}^{0}(\bar{g})-(k+1)<\operatorname{dim}^{0}(\bar{g})-$

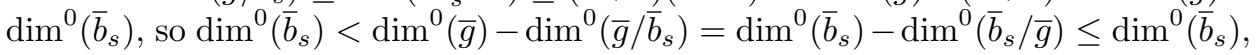
which is a contradiction.

So $\left|W_{s}(\mathbb{C}) \cap \exp _{G}\left(X_{0, s}\right)\right|=\left|\exp _{G}^{-1}\left(W_{s}(\mathbb{C})\right) \cap X_{0, s}\right| \leq\left|\mathcal{O}_{s}\right|^{k}=\left(\left|\mathcal{O}_{s}\right|^{s}\right)^{\frac{k}{s}}=$ $\left|X_{0, s}\right|^{\frac{k}{s}}$. So for any $\epsilon \in \mathbb{R}_{>0}$, considering sufficiently large $s$, we deduce $\boldsymbol{\delta}(W(K) \cap$ $\left.\exp _{G}\left(X_{0}\right)\right) \leq \epsilon \boldsymbol{\delta}\left(X_{0}\right)$. Hence $\boldsymbol{\delta}\left(W(K) \cap \exp _{G}\left(X_{0}\right)\right)=0$, as required.

By Fact 2.4 we can pick $\bar{a} \in H \cap \exp _{G}(Z)^{n}$ with $\boldsymbol{\delta}(\bar{a})=\boldsymbol{\delta}\left(H \cap \exp _{G}(Z)^{n}\right)$. By injectivity of $\exp _{G}$ on $Z$ this is $\geq \boldsymbol{\delta}\left(\operatorname{Lie}(H) \cap Z^{n}\right)=m d$. Note that $\boldsymbol{\delta}\left(a_{i}\right) \leq \boldsymbol{\delta}(Z)=d$ and that, by the above Claim $a_{i} \in \exp _{G}\left(X_{0}\right)$ is $d c g p$ in $G$ (see Def. 7.3) and hence cgp (see Lemma 7.2). So by Lemma $5.12 \bar{a}$ is coherent.

Remark 7.13. The only essential role played by Lie theory in the above proof is to establish that $F$ embeds in a matrix algebra; for the rest of the proof, $\exp _{G}$ : $\operatorname{Lie}(G) \rightarrow G$ is used only to pick out choices of systems of division points of elements of $G$, and this can instead be done directly by replacing $\exp _{G}$ with $\rho: \widehat{G} \rightarrow G$ where $\widehat{G}$ is the "profinite cover" $\widehat{G}:=\lim ([n]: G \rightarrow G)$ consisting of "division systems" $\left(x_{n}\right)_{n}$ satisfying $[n] x_{n m}=x_{m}$, and $\rho$ is the first co-ordinate map of the inverse limit, $\rho\left(\left(x_{n}\right)_{n}\right):=x_{1}$. Then $\operatorname{End}^{0}(G)$ acts on $\widehat{G}$ by $\frac{\eta}{m}\left(x_{n}\right)_{n}:=\left(\eta x_{n m}\right)_{n}$. 
Remark 7.14. In the case that $G$ is a semiabelian variety, we can do slightly better and obtain approximate $\mathcal{O}$-modules which are in general position in the sense of Elekes-Szabó rather than merely in coarse general position. More precisely, say an internal subset $X \subset G(K)$ is in general position if it has finite intersection with any proper closed subvariety $W \subsetneq G$ over $K$. Then proceed as in the proof of Proposition 7.10 but taking $\bar{\gamma}_{s}:=\gamma \in G(\mathbb{C})$ to be a singleton which is in no proper algebraic subgroup of $G$. Let $\Gamma:=\mathcal{O} \gamma \leq G(\mathbb{C})$ be the $\mathcal{O}$-submodule generated by $\gamma$, which is a finitely generated subgroup of $G(\mathbb{C})$. As shown in [Sca04, Theorem 4.7], as a consequence of the truth of the Mordell-Lang conjecture, if $V_{b}$ is a constructible family of subvarieties of $G$ then there is a uniform bound on the sizes of finite intersections $V_{b} \cap \Gamma$. Hence $\exp _{G}\left(X_{k}\right)$ is in general position in $G$.

However, this approach clearly fails for $G=\mathbb{G}_{a}^{2}$, by considering intersections with linear subvarieties. Pach [Pac03, Theorem 2] gives an example of an internal subset $X \subset K^{2}$ with $\boldsymbol{\delta}(X)=1=\boldsymbol{\delta}(X+X)$ and where the intersection with any linear subvariety has size at most 2 ; however, quadratic subvarieties witness that this $X$ is not in general position. We do not know whether it is possible to find such an $X$ which is in general position. This prompts the following question.

Question 7.15. Is there a sequence of finite sets $A_{n} \subset \mathbb{C}^{2}$ such that $\left|A_{n}\right| \rightarrow+\infty$, $\left|A_{n}+A_{n}\right| \leq\left|A_{n}\right|^{1+1 / n}$ and with the property that, for each degree $d$,

$$
\sup _{n, \mathcal{C}, \operatorname{deg} \mathcal{C} \leq d}\left|A_{n} \cap \mathcal{C}\right|
$$

is finite, where $\mathcal{C}$ runs through algebraic curves $\mathcal{C} \subset \mathbb{C}^{2}$.

7.2. Proofs of the main results. We first observe that Theorem 1.4 is a special case of Theorem 1.11, as follows immediately from the following lemma.

Lemma 7.16. Let $G$ be a 1-dimensional connected complex algebraic group, let $n \geq 1$, and let $H \leq G^{n}$ be a connected algebraic subgroup. Then $H$ is a special subgroup of $G^{n}$.

Proof. First, suppose $G$ is the additive group $(\mathbb{C} ;+)$. Then $F:=\operatorname{End}^{0}(G)=\mathbb{C}$ is a division ring, and $H$ is a vector subgroup, i.e. $H=\operatorname{ker}(A)$ for some $A \in \operatorname{Mat}_{n}(\mathbb{C})$, as required.

Otherwise, $G$ is the multiplicative group or an elliptic curve. In either case, $F:=\operatorname{End}^{0}(G)$ is again a division ring, being either $\mathbb{Q}$ or a quadratic imaginary field extension of $\mathbb{Q}$. We refer to [BHP, Lemma 4.1(i)] for the fact that $H \leq G^{n}$ is the connected component of $\operatorname{ker}(A)$ for some $A \in \operatorname{Mat}_{n}(\operatorname{End}(G))$.

Proof of Theorem 1.11. Combine Lemma 7.4 with Proposition 6.1 and Proposition 7.10 .

We end this section with a proof of Corollary 1.15 from the Introduction. It is a special case of the following more precise result:

Corollary 7.17. Suppose $\left(G_{1} ; \cdot{ }_{1}\right),\left(G_{2} ; \cdot \cdot_{2}\right)$ are non-isogenous connected complex algebraic groups of the same dimension, and $\Gamma \subset G_{1} \times G_{2}$ is a generically finite algebraic correspondence. Then there are $\tau, \epsilon, c>0$ such that if $A_{i} \subset G_{i}$ are finite sets such that $\Gamma \cap\left(A_{1} \times A_{2}\right)$ is the graph of a bijection between $A_{1}$ and $A_{2}$, and if $A_{i} \subset G_{i}$ and $A_{i} \cdot{ }_{i} A_{i} \subset G_{i}$ are $\tau$-cgp for $i=1,2$, then

$$
\max \left(\left|A_{1} \cdot A_{1}\right|,\left|A_{2} \cdot{ }_{2} A_{2}\right|\right) \geq c\left|A_{1}\right|^{1+\epsilon} .
$$

Remark 7.18. The cgp condition holds trivially for any $A$ when $\operatorname{dim}\left(G_{i}\right)=1$.

Proof. Let $V=\left\{\left(x_{1}, y_{1}, x_{1} \cdot y_{1}, x_{2}, y_{2}, x_{2} \cdot y_{2}\right):\left(x_{1}, x_{2}\right),\left(y_{1}, y_{2}\right) \in \Gamma\right\}$. Suppose $V$ is special. Then $V$ is in co-ordinatewise correspondence with a special subgroup 
$H \leq G^{6}$, say. As in Remark 1.13, the projection of $H$ to the first three co-ordinates is in co-ordinatewise correspondence with the graph $\{(x, y, x+y)\}$ of the group operation of $G$. Hence the graph of the group operation of $G_{1}$ is in co-ordinatewise correspondence with that of $G$. By Fact 2.13, $G_{1}$ is commutative and isogenous to $G$. Similarly, considering the projection to the last three co-ordinates, $G_{2}$ is commutative and isogenous to $G$. Since isogeny is an equivalence relation, this contradicts the assumption that $G_{1}$ and $G_{2}$ are not isogenous.

So by Theorem 1.11, $V$ admits a power-saving, say by $\epsilon^{\prime}$. So for sufficiently large $\tau$, if $A_{i}$ are as in the statement, then setting $X:=\left\{\left(a_{1}, b_{1}, a_{1} \cdot{ }_{1} b_{1}, a_{2}, b_{2}, a_{2} \cdot 2\right.\right.$ $\left.\left.b_{2}\right): a_{i}, b_{i} \in A_{i} ;\left(a_{1}, a_{2}\right),\left(b_{1}, b_{2}\right) \in \Gamma\right\} \subset V$, we have $\left|A_{1}\right|^{2}=|X| \leq O\left(\max \left(\mid A_{1} \cdot 1\right.\right.$ $\left.\left.A_{1}|,| A_{2} \cdot A_{2} \mid\right)^{2-\epsilon^{\prime}}\right)$. So $\epsilon:=\frac{\epsilon^{\prime}}{2-\epsilon^{\prime}}$ is as required.

Question 7.19. Consider the following weakening of coarse general position. Say $a \in K^{<\infty}$ with $\boldsymbol{\delta}(a)=\mathrm{d}^{0}(a)$ is in Larsen-Pink general position if for any $B \subset K^{<\infty}$, we have $\boldsymbol{\delta}(a / B) \leq \mathrm{d}^{0}(a / B)$. This hypothesis suffices to give the trivial upper bound of Lemma 7.1, and it is not satisfied by the counterexample of Section 4, nor by similar constructions based on nilpotent groups.

Does Theorem 1.11 go through unchanged if coarse general position is relaxed to Larsen-Pink general position?

The techniques of this paper are insufficient to prove this generalisation, as the corresponding weakened notion of coherence does not directly yield a pregeometry.

Since type-definable subgroups of simple algebraic groups do satisfy this LarsenPink condition (see [Hru13, 2.15]), a positive answer should suffice to recover the characteristic 0 case of the main theorem of [BGT11], Theorem 5.5.

\section{Coherence in SubGroups}

In this section we observe a strengthening of our results in the special case of a $\bigwedge$-definable pseudo-finite subgroup and derive Theorem 1.17 from the introduction. We then briefly discuss connections to Diophantine problems and Manin-Mumford.

Theorem 8.1. We keep the notational setup of Section 2. Let $G$ be a commutative algebraic group over $C_{0}$ and $\Gamma \leq G(K)$ be a $\bigwedge$-definable (over $\emptyset$ ) subgroup of $G(K)$ contained in a cgp definable (over $\emptyset$ ) subset of $G$ (see Definition 7.3). Assume $\boldsymbol{\delta}(\Gamma)=\operatorname{dim}(G)$. Then the locus locus ${ }^{G^{n}}\left(\bar{\gamma} / C_{0}\right)$ of any coherent tuple $\bar{\gamma} \in \Gamma^{n}$ is a coset of an algebraic subgroup.

Remark 8.2. The commutative case is the only relevant case: by Corollary 3.15, if $G$ is a connected algebraic group with such a subgroup $\Gamma$ then $G$ is commutative.

Proof. By Lemma 7.2 any $\alpha \in \Gamma$ is cgp. In particular if $\boldsymbol{\delta}(\alpha)>0$, then $\alpha$ is generic in $G$ and $\boldsymbol{\delta}(\alpha) \leq \boldsymbol{\delta}(\Gamma)=\operatorname{dim}(G)=\mathrm{d}^{0}(\alpha)$. So for all $\alpha \in \Gamma$ we have $\boldsymbol{\delta}(\alpha) \leq \mathrm{d}^{0}(\alpha)$.

By Fact 2.4, we may find $\bar{\alpha} \in \Gamma^{n}$ with $\boldsymbol{\delta}(\bar{\alpha} / \bar{\gamma})=\boldsymbol{\delta}\left(\Gamma^{n}\right)=n \operatorname{dim}(\bar{G})$. Then $\boldsymbol{\delta}(\bar{\gamma}, \bar{\alpha}, \bar{\gamma}+\bar{\alpha})=\boldsymbol{\delta}(\bar{\gamma}, \bar{\alpha})=\boldsymbol{\delta}(\bar{\gamma})+n \operatorname{dim}(G)=\mathrm{d}^{0}(\bar{\gamma})+n \operatorname{dim}(G) \geq \mathrm{d}^{0}(\bar{\gamma}, \bar{\alpha})=$ $\mathrm{d}^{0}(\bar{\gamma}, \bar{\alpha}, \bar{\gamma}+\bar{\alpha})$, and $\gamma_{i}, \alpha_{i}, \gamma_{i}+\alpha_{i} \in \Gamma$, so $(\bar{\gamma}, \bar{\alpha}, \bar{\gamma}+\bar{\alpha})$ is coherent by Lemma 5.12 .

Since the product of cosets is a coset, we may assume that locus ${ }^{G^{n}}\left(\bar{\gamma} / C_{0}\right)$ is not a product of loci of subtuples. Then as in Proposition 6.1, there is a commutative algebraic group $G^{\prime}$ over $C_{0}$ and a tuple $\left(\bar{\gamma}^{\prime}, \bar{\alpha}^{\prime}, \bar{\psi}^{\prime}\right)$ which is generic in a connected subgroup $H \leq G^{\prime 3 n}$ and which is co-ordinatewise $\operatorname{acl}^{0}$-interalgebraic with $(\bar{\gamma}, \bar{\alpha}, \bar{\gamma}+$ $\bar{\alpha})$. Furthermore, for each $i$ we have $\theta_{i}^{1} \gamma_{i}^{\prime}+\theta_{i}^{2} \alpha_{i}^{\prime}+\theta_{i}^{3} \psi_{i}^{\prime} \in G^{\prime}\left(C_{0}\right)$ for some $\theta_{i}^{j} \in$ End $_{C_{0}}\left(G^{\prime}\right)$ invertible in $\operatorname{End}_{C_{0}}^{0}\left(G^{\prime}\right)$, and so we may assume without loss that $\psi_{i}^{\prime}=$ $\gamma_{i}^{\prime}+\alpha_{i}^{\prime}$.

Then by Fact 2.13, there are $m \in \mathbb{N}$ and isogenies $\eta_{i}: G^{\prime} \rightarrow G$ and $k_{i} \in$ $G\left(C_{0}\right)$ such that $\eta_{i}\left(\gamma_{i}^{\prime}\right)=m \gamma_{i}+k_{i}$ for $i=1, \ldots, n$. Hence locus ${ }^{G^{n}}\left(m \bar{\gamma} / C_{0}\right)=$ $\left(\prod_{i} \eta_{i}\right)\left(\operatorname{locus}^{G^{n}}\left(\bar{\gamma}^{\prime}\right)\right)-\bar{k}=\left(\prod_{i} \eta_{i}\right)\left(\pi_{1}(H)\right)-\bar{k}$ where $\bar{k}=\left(k_{1}, \ldots, k_{n}\right)$ and $\pi_{1}$ : 
$(\bar{x}, \bar{y}, \bar{z}) \mapsto \bar{x}$. So locus ${ }^{G^{n}}\left(m \bar{\gamma} / C_{0}\right)$ is a coset of an algebraic subgroup of $G^{n}$, and hence so is locus $G^{n}\left(\bar{\gamma} / C_{0}\right)$, as required.

Proof of Theorem 1.1\%. First, note that there exists a function $f: \mathbb{N} \rightarrow \mathbb{N}$ such that any translate of a subvariety of $G^{n}$ of complexity $\leq \tau$ has complexity $\leq f(\tau)$. This follows from [BGT11, Lemma 3.4], and can also be seen as a consequence of the fact that the family of translates of subvarieties in a constructible family is constructible. Increasing $f$ if necessary, we may assume also that $f$ is strictly increasing and $2^{-\tau}+\frac{1}{f(\tau)} \leq \frac{1}{\tau}$ for any $\tau \in \mathbb{N}$.

By [TV06, Proposition 2.26], there are $C_{1}, C_{2}>0$ such that if $|A+A| \leq|A|^{1+\epsilon}$ then $H:=A-A$ is an $|A|^{C_{1} \epsilon}$-approximate subgroup and $|H| \leq|A|^{1+C_{2} \epsilon}$.

It therefore suffices to prove the following revised statement: there are $N, \tau, \epsilon, \eta>$ 0 depending only on $G$ and the complexity of $V$ such that if $H \subset G$ is a $\tau$-cgp $|H|^{\epsilon}$ approximate subgroup and $|H| \geq N$, then $\left|H^{n} \cap V\right|<|H|^{\frac{\operatorname{dim}(V)}{\operatorname{dim}(G)}-\eta}$. Indeed, given $\tau^{\prime} \in \mathbb{N}$, if $(N, \tau, \epsilon, \eta)$ are as required in the revised statement for $V$ of complexity at most $f\left(\tau^{\prime}\right)$, then $\left(N, \tau, \epsilon^{\prime}:=\frac{\epsilon}{C}{ }_{1}, \eta^{\prime}:=\frac{\operatorname{dim}(V)}{\operatorname{dim}(G)}-\left(\frac{\operatorname{dim}(V)}{\operatorname{dim}(G)}-\eta\right)\left(1+C_{2} \epsilon^{\prime}\right)\right)$ are as required in the original statement for $V$ of complexity at most $\tau^{\prime}$, after increasing $C_{1}$ if necessary to ensure $\eta^{\prime}>0$. Indeed, given $V$ of complexity at most $\tau^{\prime}$, suppose there is $A \subset G$ with $|A| \geq N$ and $\left|A^{n} \cap V\right| \geq|A|^{\frac{\operatorname{dim}(V)}{\operatorname{dim}(G)}-\eta^{\prime}}$ and $|A+A| \leq|A|^{1+\epsilon^{\prime}}$, and with $H:=A-A$ being $\tau$-cgp. Then $H$ is an $|A|^{C_{1} \epsilon^{\prime}}=|A|^{\epsilon}$-approximate subgroup, and $|H| \geq|A| \geq N$. Let $x \in A$, and let $V^{\prime}:=V-(x, x, \ldots, x)$, which has complexity at most $f\left(\tau^{\prime}\right)$. Then $\left|H^{n} \cap V^{\prime}\right| \geq\left|A^{n} \cap V\right| \geq|A|^{\frac{\operatorname{dim}(V)}{\operatorname{dim}(G)}-\eta^{\prime}} \geq$ $|H|^{\left(\frac{\operatorname{dim}(V)}{\operatorname{dim}(G)}-\eta^{\prime}\right)\left(1+C_{2} \epsilon^{\prime}\right)^{-1}} \geq|H|^{\frac{\operatorname{dim}(V)}{\operatorname{dim}(G)}-\eta}$. This contradicts the revised statement.

Now suppose the revised statement fails. Then there is a family $\left(V_{b}\right)_{b}$ of bounded complexity subvarieties of $G^{n}$, such that for each $s \in \mathbb{N}$ there is an $f(s)$-cgp subset $H_{s} \subset G$ such that $H_{s}$ is an $\left|H_{s}\right|^{4^{-s}}$-approximate subgroup, and a parameter $b_{s}$, such that $\left|H_{s}\right| \geq s$ and $\left|H_{s}^{n} \cap V_{b_{s}}\right| \geq\left|H_{s}\right|^{\frac{\operatorname{dim}\left(V_{b_{s}}\right)}{\operatorname{dim}(G)}-\frac{1}{s}}$ and $V_{b_{s}}$ is not a coset.

Let $\mathcal{U}$ be a non-principal ultrafilter on $\mathbb{N}$, let $\Gamma_{i}:=\prod_{s \rightarrow \mathcal{U}} \sum_{j=1}^{2^{s-i}} H_{s}$ for $i \in \mathbb{N}$, and let $\Gamma:=\bigcap_{i>0} \Gamma_{i}$. Set the language $\mathcal{L}$ to be such that each $\Gamma_{i}$ is definable. Then $\Gamma$ is a $\bigwedge$-definable subgroup, since $\Gamma_{i+1}+\Gamma_{i+1} \subset \Gamma_{i}$. Now $\boldsymbol{\delta}\left(\Gamma_{i}\right)=\boldsymbol{\delta}\left(\prod_{s \rightarrow \mathcal{U}} H_{s}\right)$ for any $i$, because $\left|\sum_{j=1}^{2^{s-i}} H_{s}\right| \leq\left|H_{s}\right|^{1+2^{s-i} 4^{-s}} \leq\left|H_{s}\right|^{1+2^{-s}}$. So $\boldsymbol{\delta}(\Gamma)=\boldsymbol{\delta}\left(\prod_{s \rightarrow \mathcal{U}} H_{s}\right)$, so setting our scaling parameter $\xi$ appropriately, we may ensure $\boldsymbol{\delta}(\Gamma)=\operatorname{dim}(G)$.

We claim that $\Gamma_{0}$ is cgp in $G$. Indeed, $\sum_{j=1}^{2^{s}} H_{s}$ is contained in the union of $\left|H_{s}\right|^{2^{s} 4^{-s}}=\left|H_{s}\right|^{2^{-s}}$ translates of $H_{s}$, so if $W \subsetneq G$ has complexity $\leq s$ then, using the lower bound we assumed on $f$, we have $\left|W \cap \sum_{j=1}^{2^{s}} H_{s}\right| \leq\left|H_{s}\right|^{2^{-s}}\left|H_{s}\right|^{\frac{1}{f(s)}} \leq$ $\left|H_{s}\right|^{\frac{1}{s}}$. So $\sum_{j=1}^{2^{s}} H_{s}$ is $s$-cgp in $G$.

Let $b:=\lim _{s \rightarrow \mathcal{U}} b_{s}$. Then $\boldsymbol{\delta}\left(\Gamma^{n} \cap V_{b}\right)=\operatorname{dim}\left(V_{b}\right)$. Set $C_{0}$ such that $G$ and $V_{b}$ are over $C_{0}$. By Fact 2.4 we can pick $\bar{\gamma}=\left(\gamma_{1}, \ldots, \gamma_{n}\right) \in \Gamma^{n} \cap V_{b}$ with $\boldsymbol{\delta}(\bar{\gamma})=$ $\boldsymbol{\delta}\left(\Gamma^{n} \cap V_{b}\right)$. Since $\Gamma_{0}$ is cgp in $G$, Lemma 7.2 implies that all $\gamma_{i}$ 's are cgp and $\boldsymbol{\delta}\left(\gamma_{i}\right) \leq \mathrm{d}^{0}\left(\gamma_{i}\right)$. Then $\bar{\gamma}$ is coherent by Lemma 5.12. So by Theorem 8.1 we have that $V_{b}=\operatorname{locus}^{G^{n}}\left(\bar{\gamma} / C_{0}\right)$ is a coset. But then so is $V_{b_{m}}$ for $\mathcal{U}$-many $m$, contradicting our assumption.

Example 8.3 (Connections with Manin-Mumford and Mordell-Lang). Let $G$ be a complex elliptic curve. Write $G[\infty]:=\bigcup_{r \in \mathbb{N}} G[r]$ for the torsion subgroup. Suppose $V \subset G^{n}$ is an irreducible closed complex subvariety such that $V(\mathbb{C}) \cap G[\infty]$ is Zariski dense in $V$.

We know, by the Manin-Mumford conjecture proven by Raynaud, that $V$ is a coset of an algebraic subgroup. Some co-ordinate projection to $G^{\operatorname{dim}(V)}$ yields an 
isogeny, so $V=H+\alpha$ where $H=\eta\left(G^{\operatorname{dim}(V)}\right)$ is a subgroup and $\eta$ is an isogeny, and $\alpha \in G[\infty]^{n}$. Setting $c:=|\operatorname{ker}(\eta)|^{-1}$ it follows that for $r \geq N:=\operatorname{ord}(\alpha)$ we have $\left|V(\mathbb{C}) \cap G[r !]^{n}\right| \geq c \cdot\left|G^{\operatorname{dim}(V)}[r !]\right|$, and so for $r \in \mathbb{N}$ we have the lower bound $\left|V(\mathbb{C}) \cap G[r !]^{n}\right| \geq \Omega(|G[r !]| \operatorname{dim}(V))$.

Suppose conversely that we only know this consequence of Manin-Mumford on the asymptotics of the number of torsion points in $V$, or even just that for every $\epsilon>0$, for arbitrarily large $r \in \mathbb{N}$ we have $\left|V(\mathbb{C}) \cap G[r !]^{n}\right| \geq|G[r !]| \operatorname{dim}(V)-\epsilon$. Then it follows that $V$ is a coset of an algebraic subgroup. Indeed, $G[r !]$ is a subgroup and is trivially $\tau$-cgp for any $\tau$ since $\operatorname{dim}(G)=1$, so this is an immediate consequence of Theorem 1.17.

We can generalise this argument by replacing $G[\infty]$ with a finite rank subgroup, as in the Mordell-Lang conjecture. Indeed, let $\Gamma \leq G(\mathbb{C})$ be a finite rank $\operatorname{End}(G)$ submodule. Say $\Gamma$ is contained in the divisible hull of the subgroup generated by $\gamma_{1}, \ldots, \gamma_{k}$. Let $\Gamma_{r}:=\left\{x \in \Gamma:(r !) x \in \sum_{i}[-r, \ldots, r] \gamma_{i}\right\}$. Then $\Gamma_{r}$ is finite and $\left|\Gamma_{r}+\Gamma_{r}\right| \leq 2^{k}\left|\Gamma_{r}\right|$, so as above we obtain that if $V \subset G^{n}$ is an irreducible closed subvariety, then $V$ is a coset of a subgroup if and only if for all $\epsilon>0$, for arbitrarily large $r \in \mathbb{N}$, we have $\left|V(\mathbb{C}) \cap \Gamma_{r}^{n}\right| \geq\left|\Gamma_{r}\right|^{\operatorname{dim}(V)-\epsilon}$.

\section{Appendix A. Projective geometries fully embedded in Algebraic GEOMETRY}

[EH91] characterises the projective subgeometries of the geometry of algebraic closure in an algebraically closed field $K$ over an algebraically closed subfield $C_{0}$. The points of such a geometry are $C_{0}$-interalgebraicity classes of elements of $K$.

In this essentially self-contained appendix, we consider the more general situation of a projective geometry induced from field-theoretic algebraic dependence whose points are $C_{0}$-interalgebraicity classes of finite tuples from $K$ (or, equivalently, of $K$-rational points of arbitrary varieties over $K$ ).

The arguments are generalisations of those used in [EH91]. We use Hrushovski's abelian group configuration theorem to find an abelian algebraic group, then apply a version of the fundamental theorem of projective geometry to identify the co-ordinatising skew field of the geometry as a skew field of quasi-isogenies of the group. Identifying the isogenies involved requires a little more care in the higherdimensional case, as there may be non-trivial endomorphisms which are not isogenies, and these cannot appear in the co-ordinatising skew field.

We allow ourselves to simplify some of the algebra by restricting ourselves to the characteristic 0 case, whereas [EH91] works in arbitrary characteristic.

Let $K$ be an algebraically closed field of characteristic 0 . Let $C_{0} \leq K$ be an algebraically closed subfield, and let $\mathrm{cl}: \mathcal{P}\left(K^{<\infty}\right) \rightarrow \mathcal{P}\left(K^{<\infty}\right)$ be field-theoretic algebraic closure over $C_{0}$ as defined in Example 5.3. In other words, for a subset $B \subset K^{<\infty}:=\bigcup_{n \geq 1} K^{n}$ we let $\operatorname{cl}(B)$ be the set of tuples from the field-theoretic algebraic closure $\bar{C}_{0}(B)^{\text {alg }}$ of the subfield $C_{0}(B) \leq K$ generated by $C_{0}$ and the co-ordinates of all tuples from $B$. This closure operator ${ }^{1}$ was denoted $\operatorname{acl}^{0}(B)<\infty$ in Section 5. So $a \in \operatorname{cl}(B)$ if and only if $a$ has finite orbit under $\operatorname{Aut}\left(K / C_{0}(B)\right)$, if and only if $a \in\left(C_{0}(B)^{\mathrm{alg}}\right)<\omega$.

If $V$ is an algebraic variety over $C_{0}$ and $a \in V(K)$ is a $K$-rational point, we may consider $a$ as a tuple in $K^{<\infty}$ as explained in $\S 2.1 .10$.

Let $\mathcal{G}_{K}:=\mathbb{P}\left(K^{<\infty} ; \mathrm{cl}\right)$ be the projectivisation of the closure structure $\left(K^{<\infty} ; \mathrm{cl}\right)$, as defined in $\S 5.1$; i.e. $\mathcal{G}_{K}=\left\{\operatorname{cl}(\{a\}): a \in K^{<\infty} \backslash \operatorname{cl}(\emptyset)\right\}$ with the closure induced

\footnotetext{
${ }^{1}$ In model theory this is usually denoted by acl ${ }^{\text {eq }}$ and is defined on subsets of $K^{\text {eq }}$, which we identify here with $K^{<\omega}$ via elimination of imaginaries.
} 
from (and still denoted by) cl. For $x \in K^{<\infty}$ and $C \subset K^{<\infty}$, define $\widetilde{x}:=\operatorname{cl}(\{x\})$ and $\widetilde{C}:=\{\widetilde{c}: c \in C\}$.

As already noted $\mathcal{G}_{K}$ is not a geometry in general (it does not satisfy the exchange property), but here we are interested in geometries that embed in $\mathcal{G}_{K}$. We say that a geometry $P$ is connected if any two points $a, b$ are non-orthogonal, i.e. if there exists $C \subset P$ such that $a \in \operatorname{cl}(b, C) \backslash \operatorname{cl}(C)$.

Lemma A.1. Let $P \subset \mathcal{G}_{K}$ and suppose that the restriction $(P, \mathrm{cl})$ of $\mathrm{cl}$ to $P$ forms a connected geometry (embedded in $\mathcal{G}_{K}$ ). Then the following are equivalent:

(i) for any $\widetilde{x} \in P$ and $\widetilde{C} \subset P, \widetilde{x} \in \operatorname{cl}(\widetilde{C}) \Leftrightarrow x \mathbb{L}_{C_{0}} C$

(ii) There exists $k \in \mathbb{N}$ such that for any finite subset $\widetilde{A} \subset P$,

$$
k \cdot \operatorname{dim}_{\mathrm{cl}}(\widetilde{A})=\mathrm{d}^{0}(A),
$$

where recall $\mathrm{d}^{0}(A):=\operatorname{trd}\left(C_{0}(A) / C_{0}\right)$.

Proof. Note that (i) is equivalent to say that $\mathrm{d}^{0}(x / C)=0$ if and only if $\mathrm{d}^{0}(x / C)<$ $\mathrm{d}^{0}(x)$. That (i) implies (ii) follows from additivity of dimensions, setting $k:=\mathrm{d}^{0}(a)$ for any $a \in K^{<\infty}$ such that $\widetilde{a} \in P$, once we see that this does not depend on the choice of $a$. For another such $b$ with $\widetilde{b} \in P$ we show that $\mathrm{d}^{0}(a)=\mathrm{d}^{0}(b)$. If $\widetilde{b}=\widetilde{a}$ then $b$ and $a$ are interalgebraic over $C_{0}$, so this holds. Else by non-orthogonality there is $\widetilde{C}$ such that $\widetilde{a} \in \operatorname{cl}(\widetilde{C} \widetilde{b})$ and $\widetilde{b} \in \operatorname{cl}(\widetilde{C} \widetilde{a})$ but $\widetilde{a}, \widetilde{b} \notin \operatorname{cl}(\widetilde{C})$. Then by (i), $\mathrm{d}^{0}(a)=\mathrm{d}^{0}(a / C)=\mathrm{d}^{0}(b / C)=\mathrm{d}^{0}(b)$. The converse is easy and since it is not needed in the sequel, we leave it to the reader.

Definition A.2. We say a connected geometry $(P, \mathrm{cl}) \subset \mathcal{G}_{K}$ is (k-dimensionally) fully embedded in $\mathcal{G}_{K}$ if the equivalent conditions of the above lemma hold.

If $G$ is a connected abelian algebraic group over $C_{0}$, let $E_{G}:=\operatorname{End}_{C_{0}}(G)$ be the ring of algebraic endomorphisms defined over $C_{0}$, and let $E_{G}^{0}:=\operatorname{End}_{C_{0}}^{0}(G):=$ $\mathbb{Q} \otimes_{\mathbb{Z}} E_{G}$. Any $\eta \in E_{G}^{0}$ can be written as $q \eta^{\prime}$ for some $q \in \mathbb{Q}$ and $\eta^{\prime} \in E_{G}$. Since $\operatorname{char}(K)=0$ and $G$ is connected, $G(K)$ is divisible, and the $n$-torsion is finite for all $n$ and hence contained in $G\left(C_{0}\right)$. So $V:=G(K) / G\left(C_{0}\right)$ is naturally a left $E_{G}^{0}$-module. If $F \leq E_{G}^{0}$ is a division subring, we view $V$ as an $F$-vector space and let $\mathbb{P}_{F}(G):=\mathbb{P}(V)$ be its projectivisation, and let $\eta_{F}^{G}: \mathbb{P}_{F}(G) \rightarrow \mathcal{G}_{K}$ be the map induced by $g \mapsto \widetilde{g}$ for $g \in G(K)$. Note that $\eta_{F}^{G}$ is not injective.

Example A.3. Let $G$ and $F$ be as above. Let $g_{i} \in G(K)$ be independent generics over $C_{0}$ for $i$ in a (possibly infinite) index set $I$. Let $V:=\left\langle\left(g_{i} / G\left(C_{0}\right)\right)_{i \in I}\right\rangle_{F} \leq$ $G(K) / G\left(C_{0}\right)$. Then $\eta_{F}^{G}$ maps the $|I|$-dimensional projective geometry $\mathbb{P}_{F}(V) \subset$ $\mathbb{P}_{F}(G)$ injectively into $\mathcal{G}_{K}$, and the image $\eta_{F}^{G}\left(\mathbb{P}_{F}(V)\right)$ is $\operatorname{dim}(G)$-dimensionally fully embedded in $\mathcal{G}_{K}$.

For example, in the case $G=\mathbb{G}_{m}$, if $a_{0}, \ldots, a_{n} \in K$ with $\operatorname{trd}\left(\bar{a} / C_{0}\right)=n+1$, then they generate in $K^{*} / C_{0}^{*}$ the $\mathbb{Q}$-subspace $\left\{\bar{a}^{\bar{q}} / C_{0}^{*}: \bar{q} \in \mathbb{Q}^{n+1}\right\}=\left\{\prod_{i} a_{i}^{q_{i}} / C_{0}^{*}\right.$ : $\left.q_{0}, \ldots, q_{n} \in \mathbb{Q}\right\}$; the algebraic dependencies over $C_{0}$ within this set are precisely those arising from $\mathbb{Q}$-linear dependencies on the exponents, and so this yields an embedding of $\mathbb{P}^{n}(\mathbb{Q})$ in $\mathcal{G}_{K}$.

The following proposition, which is the main result of this appendix, says that any fully embedded projective geometry (of sufficiently large dimension) is of this form.

Proposition A.4. Let $(P, \mathrm{cl}) \subset \mathcal{G}_{K}$ be a $k$-dimensionally fully embedded geometry, and suppose $P$ is isomorphic to the geometry of a projective space over a division ring $F$, and $\operatorname{dim}(P) \geq 3$. 
Then there is an abelian algebraic group $G$ over $C_{0}$ of dimension $k$, and an embedding of $F$ as a subring $F \leq E_{G}^{0}$, and a closed subgeometry $P^{\prime}$ of $\mathbb{P}_{F}(G)$ on which $\eta_{F}^{G}$ is injective, such that $P=\eta_{F}^{G}\left(P^{\prime}\right)$.

Furthermore, $G$ is unique up to isogeny.

The remainder of this appendix constitutes a proof of Proposition A.4. The strategy of the proof is to find the commutative algebraic group $G$ via the abelian group configuration theorem, and then to exhibit a natural injective collineation from $P$ to $\mathbb{P}_{F}(G)$. The fundamental theorem of projective geometry, in its version over division rings, then allows to claim that this collineation is a projective embedding. However, since we must also identify $F$ within $E_{G}^{0}$, we will in fact use a more general form of the fundamental theorem.

Remark A.5. In fact the proof applies directly to $C_{0} \prec K$ models of an arbitrary theory of finite Morley rank, with definable groups and endomorphisms in place of algebraic groups and endomorphisms, as long as any connected definable abelian group is divisible (equivalently, has finite $n$-torsion for all $n$ ).

Remark A.6. Unlike in [EH91], our techniques do not directly apply in the case of $P$ a projective plane (i.e. a 3-dimensional connected modular geometry), and so do not rule out non-Desarguesian projective planes. However, David Evans has pointed out to us that the arguments of [Lin85] go through to show that any projective plane appearing as a subgeometry of $\mathrm{cl}$ is Desarguesian (and hence is a projective plane over a division ring).

Lemma A.7. Suppose $G$ is an abelian algebraic group over $C_{0}$, and let $g, h \in$ $G(K)$ and $b \in P$. Suppose $\widetilde{g}, \tilde{h}, b \in P$ are independent, and $\widetilde{g+h} \in P$, and $d \in \operatorname{cl}(\widetilde{g}, b) \backslash\{\widetilde{g}, b\}$.

Then there is $h^{\prime} \in G(K)$ such that $\widetilde{h^{\prime}}=b$ and $\widetilde{g+h^{\prime}}=d$.

Proof. By modularity, say $c \in \operatorname{cl}(\widetilde{h}, b) \cap \operatorname{cl}(\widetilde{g+h}, d)$. Then we proceed as in [EH95, Lemma 1.2]. Say $b=\widetilde{b}^{\prime}$ and $d=\widetilde{d}^{\prime}$. Then by the coheir property of independence in the stable theory $A C F$ any formula in $\operatorname{tp}\left(c / C_{0} b^{\prime} d^{\prime} g\right)$ is satisfiable in $C_{0}$. So since $b^{\prime}$ resp. $d^{\prime}$ is interalgebraic over $c$ with points $x$ resp. $y$ of $G$ satisfying $x+g=y$, they are already interalgebraic over $C_{0}$ with such points, as required.

(Alternatively, this argument can be phrased purely algebraically, by taking a specialisation of $c$ to $C_{0}$ fixing $b^{\prime} d^{\prime} g$; see [EH91, Lemma 2.1.1].)

Lemma A.8. Suppose $a, b \in P, a \neq b$. Then there is an abelian algebraic group $G$ over $C_{0}$ with $\operatorname{dim}(G)=k$, and there exist $g, h \in G(K)$ such that $a=\widetilde{g}$, and $b=\widetilde{h}$, and $\operatorname{cl}(a, b) \backslash\{a, b\}=P \cap\{\widetilde{g+h}: g, h \in G(K), a=\widetilde{g}, b=\widetilde{h}\}$.

Proof. This proof closely follows the argument of [EH91, Theorem 3.3.1]. As there, this proof actually only needs $\operatorname{dim}(P) \geq 3$, but we will make the argument slightly more concrete by using that $P$ is a projective geometry over $F$. 
Take $c \in P \backslash \operatorname{cl}(a, b)$, and identify $\operatorname{cl}(a, b, c)$ with $\mathbb{P}\left(F^{3}\right)$, placing $a, b, c$ at $[0: 1$ : $0],[0: 0: 1],[1: 0: 0]$ respectively. Consider the following configuration.

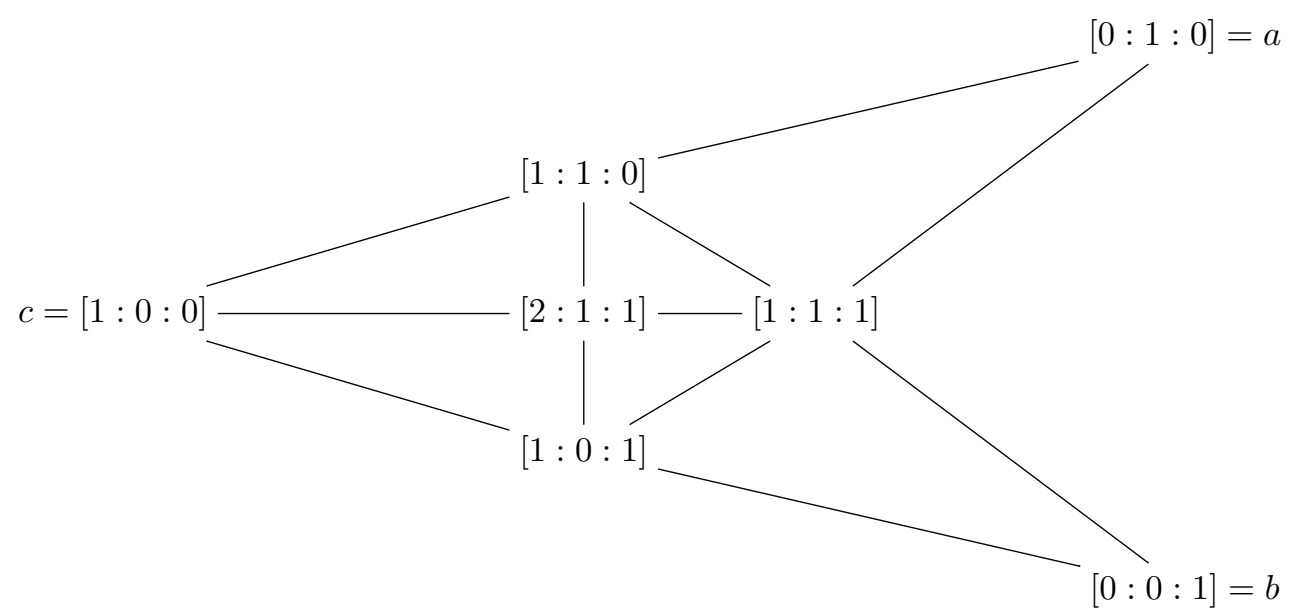

By the assumption that $P$ is fully embedded in $\mathcal{G}_{K}$, this satisfies the assumptions of Fact 3.0.1. Hence there exist an abelian algebraic group $G$ over $C_{0}$ and generics $g, k \in G(K)$ such that

$$
\begin{aligned}
a=[0: 1: 0] & =\widetilde{g} \\
c=[1: 0: 0] & =\widetilde{k} \\
{[1: 1: 0] } & =\widetilde{g+k} .
\end{aligned}
$$

Now let $d \in \operatorname{cl}(a, b) \backslash\{a, b\}$. Then by Lemma A.7 applied to $g, k, b$, we have $d=\widetilde{g+h}$ for some $h \in G(K)$ with $\widetilde{h}=b$. The converse inclusion is clear.

Now let $a_{0}, b_{0} \in P, a_{0} \neq b_{0}$, and fix $G$ and $g_{0}, h_{0} \in G(K)$ as provided by this lemma, with $\widetilde{g_{0}}=a_{0}$ and $\widetilde{h_{0}}=b_{0}$.

Say $g \in G(K)$ is $P$-aligned if there exists $g^{\prime} \in G(K)$ with $\widetilde{g^{\prime}} \neq \widetilde{g}$ such that $\widetilde{g}, \widetilde{g^{\prime}}, \widetilde{g+g^{\prime}} \in P$. Such a $g^{\prime}$ is a $P$-alignment witness for $g$.

Lemma A.9. Every point of $P$ is of the form $\widetilde{g}$ for some $P$-aligned $g \in G(K)$.

Proof. Let $c \in P$. If $c \in \operatorname{cl}\left(a_{0}, b_{0}\right)$, such a $g$ exists by Lemma A.8. Else, it follows from Lemma A.7 applied to $g_{0}, h_{0}, c$.

Lemma A.10. If $g \in G(K)$ is $P$-aligned and $b \in P \backslash \widetilde{g}$, then there exists a $P$ alignment witness $g^{\prime} \in G(K)$ for $g$ with $\widetilde{g^{\prime}}=b$.

Proof. By Lemma A.7, if $g^{\prime \prime} \in G(K)$ is a $P$-alignment witness for $g$ and $b \notin \operatorname{cl}\left(\widetilde{g}, \widetilde{g^{\prime \prime}}\right)$, then there exists a $P$-alignment witness $g^{\prime}$ for $g$ with $\widetilde{g^{\prime}}=b$.

To handle the case that $b \in \operatorname{cl}\left(\widetilde{g}, \widetilde{g^{\prime \prime}}\right)$, apply this first to $b^{\prime} \in P \notin \operatorname{cl}\left(\widetilde{g}, \widetilde{g^{\prime \prime}}\right)$ to obtain a witness $g^{\prime}$, and then again to $b \notin \operatorname{cl}\left(\widetilde{g}, \widetilde{g^{\prime}}\right)$.

Lemma A.11. If $g, h \in G(K)$ are P-aligned and $\widetilde{g}=\widetilde{h}$, then $E_{G}^{0}\left(g / G\left(C_{0}\right)\right)=$ $E_{G}^{0}\left(h / G\left(C_{0}\right)\right)$.

Proof. Say $g^{\prime}, h^{\prime} \in G(K)$ are $P$-alignment witnesses for $g, h$ respectively.

By Lemma A.10, we may assume $\widetilde{g^{\prime}} \notin \operatorname{cl}\left(\widetilde{h}, \widetilde{h^{\prime}}\right)$.

By Lemma A.7, there is $h^{\prime \prime} \in G(K)$ such that $\widetilde{h^{\prime \prime}}=\widetilde{h^{\prime}}$ and $\widetilde{g+h^{\prime \prime}}=\widetilde{h+h^{\prime}}$. Then by Fact 2.13, there is $n \in \mathbb{N}$ and an isogeny $\alpha \in E_{G}$ and $k \in G\left(C_{0}\right)$ such that $\alpha g=n h+k$, as required. 
Lemma A.12. Suppose $g, h \in G(K)$ are P-aligned and $\widetilde{g} \neq \widetilde{h}$. Then $\operatorname{cl}(\widetilde{g}, \widetilde{h})=$ $P \cap\left\{\widetilde{k}: k / G\left(C_{0}\right) \in E_{G}^{0}\left(g / G\left(C_{0}\right)\right)+E_{G}^{0}\left(h / G\left(C_{0}\right)\right)\right\}$.

Proof. By Lemma A.10, we may take a $P$-alignment witness $g^{\prime \prime}$ for $g$ with $\widetilde{h} \notin$ $\operatorname{cl}\left(\widetilde{g}, \widetilde{g^{\prime \prime}}\right)$.

Let $d \in \operatorname{cl}(\widetilde{g}, \widetilde{h}) \backslash\{\widetilde{g}, \widetilde{h}\}$. Then by Lemma A.7, $d=\widetilde{g+h^{\prime}}$ for some $h^{\prime} \in G(K)$ with $\widetilde{h^{\prime}}=\widetilde{h}$. Then $\widetilde{h^{\prime}}$ is $P$-aligned, so by Lemma A.11, $h^{\prime} / G\left(C_{0}\right) \in E_{G}^{0}\left(h / G\left(C_{0}\right)\right)$.

Our aim is to recognise $F$ as a subring of $E_{G}^{0}$, and $P$ as embedded in the corresponding $F$-projectivisation of a subspace of $G(K) / G(C)$. This is a matter of the fundamental theorem of projective geometry. However, since $E_{G}^{0}$ is not necessarily a field, this is not the classical case of the fundamental theorem. We use instead a version for projective spaces over rings obtained by Faure [Fau04].

The following definitions are adapted from [Fau04].

Definition A.13. The projectivisation $\mathbb{P}(M)$ of a module $M$ over a ring $R$ is the set of non-zero 1-generated submodules $R x$ equipped with the closure operator $\operatorname{cl}_{\mathbb{P}(M)}$ induced from $R$-linear span,

$$
\operatorname{cl}_{\mathbb{P}(M)}\left(\left(R y_{i}\right)_{i}\right):=\left\{R x: x \in\left\langle\left(y_{i}\right)_{i}\right\rangle_{R}\right\} .
$$

A point $B \in \mathbb{P}(M)$ is free if $B=R x$ for some $x \in M$ for which $\{\lambda \in R: \lambda x=$ $0\}=\{0\}$.

If $N$ is a module over a ring $S$, a map $g: \mathbb{P}(M) \rightarrow \mathbb{P}(N)$ is a projective morphism if $g\left(\operatorname{cl}_{\mathbb{P}}(M)(A)\right) \subset \operatorname{cl}_{\mathbb{P}}(N)(g(A))$ for any $A \subset \mathbb{P}(M)$.

If $\sigma: R \rightarrow S$ is a homomorphism, an additive map $f: M \rightarrow N$ is $\sigma$-semilinear if $f(\lambda x)=\sigma(\lambda) f(x)$ for any $x \in M$ and $\lambda \in R$. If $f$ is injective, $\mathbb{P}(f): \mathbb{P}(M) \rightarrow \mathbb{P}(N)$ is the induced projective morphism.

A ring $R$ is directly finite if $(\forall \lambda, \mu \in R)(\lambda \mu=1 \Rightarrow \mu \lambda=1)$.

Fact A.14. Suppose $M$ and $N$ are modules over rings $R$ and $S$ respectively, and $S$ is directly finite. Suppose $g: \mathbb{P}(M) \rightarrow \mathbb{P}(N)$ is a projective morphism, and $\operatorname{im}(g)$ contains free points $B_{1}, B_{2}, B_{3}$ such that for any $C_{1}, C_{2} \in \operatorname{im}(g)$, for some $i \in\{1,2,3\}$, we have

$$
\operatorname{cl}_{\mathbb{P}(N)}\left(C_{1}, C_{2}\right) \cap \operatorname{cl}_{\mathbb{P}(N)}\left(B_{i}\right)=\emptyset .
$$

Then there exists an embedding $\sigma: R \rightarrow S$ and a $\sigma$-semilinear embedding $f$ : $M \rightarrow N$ such that $g=\mathbb{P}(f)$.

Proof. This is the statement of [Fau04, Theorem 3.2] in the case $E=\emptyset$, except that there $C_{1}$ and $C_{2}$ are not restricted to $\operatorname{im}(g)$; however, in the proof the condition is used only when $C_{1}$ and $C_{2}$ are in $\operatorname{im}(g)$.

Let $\mathbb{P}(G):=\mathbb{P}_{E_{G}^{0}}(G)$ be the projectivisation of the $E_{G}^{0}$-module $G(K) / G\left(C_{0}\right)$.

Then Lemma A.9 and Lemma A.11 establish a map $\iota: P \hookrightarrow \mathbb{P}_{E_{G}^{0}}(G)$, which by Lemma A.12 is a projective morphism. We proceed to verify the assumptions of Fact A.14.

$E_{G}^{0}$ is directly finite since if $\mu \lambda=1$ with $\mu, \lambda \in E_{G}$ then $\mu$ is an isogeny so has a quasi-inverse $\mu^{\prime} \in E_{G}$ with $n:=\mu^{\prime} \mu \in \mathbb{N}_{>0}$; then $\lambda \mu n=n \lambda \mu=\mu^{\prime} \mu \lambda \mu=\mu^{\prime} \mu=n$, so $\lambda \mu=1$ since $G$ is $n$-divisible.

Now $\operatorname{dim}(P) \geq 3$, so say $\widetilde{g}_{1}, \widetilde{g}_{2}, \widetilde{g}_{3} \in P$ are independent with $g_{i}$ being $P$-aligned. Let $B_{i}:=\iota\left(a_{i}\right)=E_{G}^{0} g_{i}$. Since each $g_{i}$ is generic in $G(K)$, each $B_{i}$ is free. To check (C3), suppose $C_{i}=\iota\left(\widetilde{h}_{i}\right)$ for $i=1,2$ with $h_{i}$ being $P$-aligned. Then some $\widetilde{g}_{i} \notin \operatorname{cl}\left(\widetilde{h}_{1}, \widetilde{h}_{2}\right)$, and so since $P$ is fully embedded in $\mathcal{G}_{K}$, we have $g_{i} \downarrow^{0} h_{1} h_{2}$, from which (C3) follows. 
Now say $V$ is an $F$-vector space such that $P \cong \mathbb{P}(V)$. Then by Fact A.14, for some embedding $\sigma: F \rightarrow E_{G}^{0}$ and $\sigma$-semilinear embedding $f: V \rightarrow G(K) / G\left(C_{0}\right)$, we have $\iota=\mathbb{P}(f)$.

The main statement of Proposition A.4 follows.

For the uniqueness up to isogeny of $G$, suppose Proposition A.4 also holds for a group $G^{\prime}$. then if $g, h \in G(K)$ with $\widetilde{g}, \widetilde{h} \in P$ and $\widetilde{g} \neq \widetilde{h}$, then, as in the proof of Lemma A.12, there are $g^{\prime}, h^{\prime} \in G^{\prime}(K)$ with $\widetilde{g^{\prime}}=\widetilde{g}$ and $\widetilde{h^{\prime}}=\widetilde{h}$ and $\widetilde{g+h}=\widetilde{g^{\prime}+h^{\prime}}$. So by Fact $2.13, G^{\prime}$ is isogenous to $G$.

\section{REFERENCES}

[Art57] Emil Artin. Geometric algebra. Interscience Publishers, Inc., New York-London, 1957.

[BGT11] Emmanuel Breuillard, Ben Green, and Terence Tao. Approximate subgroups of linear groups. Geom. Funct. Anal., 21(4):774-819, 2011.

[BHM17] Martin Bays, Martin Hils, and Rahim Moosa. Model theory of compact complex manifolds with an automorphism. Trans. Amer. Math. Soc., 369(6):4485-4516, 2017.

[BHP] Martin Bays, Bradd Hart, and Anand Pillay. Universal covers of commutative finite morley rank groups. J. Inst. Math. Jussieu., 19:767-799, 2020.

[BMP14] Thomas Blossier and Amador Martin-Pizarro. De beaux groups. Confluentes Math., 6(1):29-39, 2014.

[BW16] Emmanuel Breuillard and Hong Wang. Erdős geometry and the group configuration. 2016. Oberwolfach report, Model Theory Workshop.

[Cam92] Peter J. Cameron. Projective and polar spaces, volume 13 of QMW Maths Notes. Queen Mary and Westfield College, School of Mathematical Sciences, London, [1992].

[CS] Artem Chernikov and Sergei Starchenko. A model-theoretic generalization of the ElekesSzabó theorem. arXiv:1801.09301 [math.LO].

[dZ18] Frank de Zeeuw. A survey of Elekes-Rónyai-type problems. In New trends in intuitive geometry, volume 27 of Bolyai Soc. Math. Stud., pages 95-124. János Bolyai Math. Soc., Budapest, 2018.

[EH91] David M. Evans and Ehud Hrushovski. Projective planes in algebraically closed fields. Proc. London Math. Soc. (3), 62(1):1-24, 1991.

[EH95] David M. Evans and Ehud Hrushovski. The automorphism group of the combinatorial geometry of an algebraically closed field. J. London Math. Soc. (2), 52(2):209-225, 1995.

[ES12] György Elekes and Endre Szabó. How to find groups? (and how to use them in Erdös geometry?). Combinatorica, 32(5):537-571, 2012.

[Fau04] Claude-Alain Faure. Morphisms of projective spaces over rings. Adv. Geom., 4(1):19-31, 2004.

[FPS ${ }^{+}$17] Jacob Fox, János Pach, Adam Sheffer, Andrew Suk, and Joshua Zahl. A semi-algebraic version of Zarankiewicz's problem. J. Eur. Math. Soc. (JEMS), 19(6):1785-1810, 2017.

[Hru12] Ehud Hrushovski. Stable group theory and approximate subgroups. J. Amer. Math. Soc., 25(1):189-243, 2012.

[Hru13] Ehud Hrushovski. On pseudo-finite dimensions. Notre Dame J. Form. Log., 54(34):463-495, 2013.

[HW08] Ehud Hrushovski and Frank Wagner. Counting and dimensions. In Model theory with applications to algebra and analysis. Vol. 2, volume 350 of London Math. Soc. Lecture Note Ser., pages 161-176. Cambridge Univ. Press, Cambridge, 2008.

[HZ96] Ehud Hrushovski and Boris Zilber. Zariski geometries. J. Amer. Math. Soc., 9(1):1-56, 1996.

[Jac75] Nathan Jacobson. PI-algebras. Lecture Notes in Mathematics, Vol. 441. SpringerVerlag, Berlin-New York, 1975. An introduction.

[Lin85] Bernt Lindström. A Desarguesian theorem for algebraic combinatorial geometries. Combinatorica, 5(3):237-239, 1985.

[Mar02] David Marker. Model theory, volume 217 of Graduate Texts in Mathematics. SpringerVerlag, New York, 2002.

[Pac03] János Pach. Midpoints of segments induced by a point set. Geombinatorics, 13(2):98$105,2003$.

[Pil96] Anand Pillay. Geometric stability theory, volume 32 of Oxford Logic Guides. The Clarendon Press Oxford University Press, New York, 1996.

[Pil98] Anand Pillay. Model theory of algebraically closed fields. In Model theory and algebraic geometry, volume 1696 of Lecture Notes in Math., pages 61-84. Springer, Berlin, 1998. 
[PS98] János Pach and Micha Sharir. On the number of incidences between points and curves. Combin. Probab. Comput., 7(1):121-127, 1998

[RSDZ16] Orit Raz, Micha Sharir, and Frank De Zeeuw. Polynomials vanishing on Cartesian products: the Elekes-Szabó theorem revisited. Duke Math. J., 165(18):3517-3566, 2016.

[RSdZ18] Orit Raz, Micha Sharir, and Frank de Zeeuw. The Elekes-Szabó theorem in four dimensions. Israel J. Math., 227(2):663-690, 2018.

[Sca04] Thomas Scanlon. Automatic uniformity. Int. Math. Res. Not., (62):3317-3326, 2004.

[She19] Adam Sheffer. Polynomial Methods and Incidence Theory. 2019. Unfinished draft, 22.08.2019.

[SSZ18] Adam Sheffer, Endre Szabó, and Joshua Zahl. Point-Curve Incidences in the Complex Plane. Combinatorica, 38(2):487-499, 2018.

[Shp08] Igor Shparlinski. On the elliptic curve analogue of the sum-product problem. Finite Fields Appl., 14(3):721-726, 2008.

[ST83] Endre Szemerédi and William T. Trotter, Jr. Extremal problems in discrete geometry. Combinatorica, 3(3-4):381-392, 1983.

[Tao15] Terence Tao. Expanding polynomials over finite fields of large characteristic, and a regularity lemma for definable sets. Contrib. Discrete Math., 10(1):22-98, 2015.

[TV06] Terence Tao and Van Vu. Additive combinatorics, volume 105 of Cambridge Studies in Advanced Mathematics. Cambridge University Press, Cambridge, 2006.

[TZ12] Katrin Tent and Martin Ziegler. A course in model theory, volume 40 of Lecture Notes in Logic. Association for Symbolic Logic, La Jolla, CA; Cambridge University Press, Cambridge, 2012.

[Wan14] Hong Wang. An application of algebraic geometry to combinatorics, a refinement of the Elekes-Szabó theorem. 2014. Masters thesis, Université Paris-Sud, Orsay.

[Zie06] Martin Ziegler. A note on generic types. 2006. arXiv:0608433v1 [math.LO].

Martin Bays, Institut für Logik und Grundlagenforschung, Fachbereich Mathematik und Informatik, Universität Münster, Einsteinstrasse 62, 48149 Münster, Germany

Email address: mbays@sdf .org

Emmanuel Breuillard, DPMms, Centre for Mathematical Sciences, Wilberforce Road, Cambridge CB3 0WB, England

Email address: ef jb2@dpmms.cam.ac.uk 